\title{
Robust 3D acoustic performance probabilistic model for nacelle liners
}

\author{
Vincent Dangla * \\ Airbus Operations S.A.S, 31300 Toulouse, France \\ Gustave Eiffel University, 77454 Marne-la-Vallée, France \\ Christian Soize ${ }^{\dagger}$ \\ Gustave Eiffel University, 77454 Marne-la-Vallée, France \\ Guilherme Cunha ${ }^{\ddagger}$, Aurélien Mosson ${ }^{\S}$, Morad Kassem ${ }^{\mathbb{I I}}$ \\ Airbus Operations S.A.S, 31300 Toulouse, France \\ Benoit Van Den Nieuwenhof ${ }^{\|}$ \\ Free Field Technologies, 1435 Mont-Saint-Guibert, Belgium
}

This paper is devoted to the robust optimization of nacelle liners (acoustic treatments). The full computational aeroacoustic model is based on the convected Helmholtz equation in presence of a non-homogeneous flow velocity field, which is computed by solving the potential Euler equations. Uncertainties are taken into account in order to increase the robustness of the predictions. A reduced-order computational aeroacoustic model is constructed in order to implement the nonparametric probabilistic model of uncertainties induced by the modeling errors in the aeroacoustic model and in the liner model. In addition, the uncertainties on the acoustic excitation induced by the fan are taken into account using the parametric probabilistic approach. The developed methodology is applied to a 3D nacelle intake and allows for computing the confidence regions of the random far-field radiated pressure in terms of random SPL (Sound Pressure Level), which are compared to experiments for one flight condition, one frequency, and for two types of liners.

\section{Nomenclature}






$p$
$\mathbf{q}$
$\mathbf{Q}$
$\mathbf{q o I}$
$\mathbf{Q o I}$
$\mathbf{r}=(x, y, z)$
$R$
$[\mathbb{Z}]$
$[\mathbf{Z}]$
$\delta_{A}$
$\delta_{Z}$
$\partial \Omega_{\mathrm{i}}, \partial \Omega_{\mathrm{o}}, \partial \Omega_{\text {duct }}$
$\varphi$
$\omega$
$\Omega_{\mathrm{i}}, \Omega_{\mathrm{o}}, \Omega_{\text {duct }}$

$=$ acoustic pressure field $(P a)$

$=$ generalized coordinates of the reduced-order model

$=$ random generalized coordinates of the stochastic reduced-order model

vector of the quantities of interest

random vector of the quantities of interest

$=$ point of coordinates $(x, y, z)$ or vector of components $(x, y, z)$

$=$ Euclidean norm of $\mathbf{r}$

$=$ reduced matrix of the liner

random reduced matrix of the liner

hyperparameter allowing for controlling the level of uncertainties in the aeroacoustic model

hyperparameter allowing for controlling the level of uncertainties in the liner model

boundaries of the inner, outer, and duct domains

acoustic velocity potential field in the duct domain

angular frequency in $\mathrm{rad} / \mathrm{s}$

$=$ inner, outer, and duct domains of the intake acoustic problem

\section{Introduction}

$I_{b}^{N}$ modern turbofan engines, fan noise is one of the main noise sources due to the constant increasing of engines bypass ratio for fuel burn reduction purposes. Since the air traffic has known a great expansion in the last decades, the environment problem became a main focus for all protagonists. In particular, aircraft noise represents a significant interest for the aeronautic community whether it is a question of ground impact or customer comfort. Moreover, the certification of aircraft is increasingly strict in terms of noise pollution. This forces engine and aircraft manufacturers to work in early phases of the aircraft development on noise reduction solutions, way before the aircraft first flight. As fan noise is characterized by broadband and tonal components, acoustic liners (treatments) are introduced for their effectiveness in mitigating both components. The tonal component is reduced by a resonance effect, while broadband noise is tackled by dissipation effects of the liners. Both dissipation effects are tuned on specific frequencies by modifying the liner geometry or intrinsic properties. In order to be efficient, namely to absorb the fan noise, liners have to be studied in their operating environment, for several flight conditions. For that purpose, simulations issued from prediction numerical tools are extensively used, since experiments cannot be considered for obvious costs reasons. A scheme of the generic 3D nacelle involving the fan, the liners, and the nacelle intake is shown in Fig. 1. The noise is produced by the fan and we are interested in predicting the far-field radiated pressure as a function of the liners acoustic properties.



Fig. 1 Scheme of a generic 3D nacelle

As the design of liner systems is frozen in early stages of an aircraft development, there exists a non-negligible variability on the operating environment. This variability directly impacts the design of liners by inducing large discrepancies on the inputs used for their design. Moreover, each time these quantities are updated due to the increased maturity of the 
aircraft program, lined surfaces are to be reoptimized in order to correctly respond to these updates, as well as verifying that the previous optimum design is still up to date. The updating phase thus represents important costs that could be avoided, in a certain measure, by accounting for such a variability in early phases of the liner design. This is the main problematic of the present paper. As this external variability directly impacts the environment of the liner, and more generally, the acoustic response of the lined system for which the flow is highly dependent on the environment (thermodynamic quantities such as the mean pressure, velocity, density of the fluid), the computational model of the liner acoustic performance is uncertain.

In order to quantify and account for such an uncertain nature, a robust design of the liner is carried out in the present paper, by quantifying the overall uncertainty that lies within the liner design computational process. The uncertainty quantification needs to be performed in a well-defined mathematical framework. The state-of-the-art computational aeroacoustic model of nacelle liners performance is an industrial numerical code, Actran/TM, which has to be analyzed so as to exhibit the main components that are subjected to uncertainties.

Once the computational model is fully defined, a stochastic modeling of uncertainties is introduced and grafted on the computational model. This stochastic modeling allows for simulating the previously mentioned external variability, by accounting for the uncertainty that lies within the model (modeling errors and model parameters errors), through nonparametric and parametric probabilistic approaches of uncertainties. Then, the propagation of uncertainties in the system is analyzed using the computational model and stochastic solver, such as the Monte Carlo method. The acoustic response is then random and the quantification of uncertainties consists in estimating statistics, such as the mean, the variance, or the confidence regions associated with a certain confidence level of quantities of interest. From this statistical information, the robustness of a given liner design towards a simulated variability on its performance model can be defined, in addition to the state-of-the-art liner acoustic performance. This information, coupled to the performance information, allows for knowing the propensity of a given liner design for maintaining its nominal performance when its environment is changing in a predefined range of variation, accounted for by the level of uncertainty imposed on the stochastic model. Then, making a compromise between performance and robustness, the liner design can be chosen. The level of uncertainty has to be identified, prior to the optimization step, using experimental data for example.

The parametric and nonparametric probabilistic approaches of uncertainties, their propagation, and their quantification solving statistical inverse problems can be found in [1]. Some works based on the use of the parametric probabilistic approach of uncertain parameters, devoted to uncertainty quantification of the liner impedance in presence of a flow have been carried out in the last decade by Jones [2] for a two-parameters liner impedance model and Brown [3] for the test bench used at NASA for liner impedance measurements. In the same framework, other research groups also worked on the uncertainty quantification of liner impedance eduction technologies such as Nark [4] and Zhou [5] and the uncertainty on liner performance as done by Robinson [6]. Nevertheless, to our knowledge, uncertainty quantification of modeling errors in liner performance aeroacoustic models has never been undertaken in the past. For that, the nonparametric probabilistic approach of modeling errors [7] presents itself as a suitable means to be used for taking into account model uncertainties.

In this paper, we present an extended version of [8], developing the mathematical and the computational model, and presenting a novel robust optimization analysis. In Section $\Pi$. we present the nominal computational model, also called mean computational model. This computational model includes the aeroacoustic model and the liner model. This mean computational model corresponds to the finite/infinite element discretization of the weak formulation of the boundary value problem (BVP) of a lined intake. Section III] deals with the construction of a computational reduced-order model that is required for reducing the numerical cost but above all, for allowing the nonparametric probabilistic approach of uncertainties to be implemented. This reduced-order model is constructed using a pellicular basis that is associated with the acoustic radiation in the acoustic layer (pellicular domain) associated with the liner dofs (degrees of freedom) [9]. In Section IV] we present the probabilistic model for uncertainty quantification. It consists in constructing a probabilistic model for the aeroacoustic model, for the liner model, and for the acoustic excitation induced by the fan. SectionVI deals with the simulated and experimental quantities of interest. Section $\square$ is devoted to the presentation of the configuration and of the computational model of a generic industrial 3D nacelle. SectionVII deals with a sensitivity analysis of modal SPL with respect to the levels of uncertainties. In Section VIII, we present the experimental identification of uncertainty levels for aeroacoustic, liner and modal content. Finally, Section IX] deals with the robust optimization of liners under uncertainties. 


\section{Construction of the nominal (mean) computational model}

\section{A. Framework of the presented methodology}

From the theory of sound generated aerodynamically written by Sir Lighthill in the 1950s [10, 11], numerous works have been undertaken since. To name a few, authors such as Landau and Lifshitz [12] and Mohring [13] contributed to the development of fluid dynamics, and Bruneau and Pierce contributed to the propagation of acoustics [14, 15]. The prediction of such physical phenomena through numerical tools have been created and constantly improved, based on their work. Authors such as Jones [16] that worked on the aeroacoustic propagation, Tam [17,-20] on computational aeroacoustic matters such as the acoustic boundary conditions, and others [21-24], have contributed to the expansion and improvement of these techniques. For such prediction numerical models, the flow behaviour inside and outside the nacelle is characterized using computational fluid dynamics tools ([25, 26]), and allows for knowing the thermodynamical state of the fluid in which the acoustic perturbation propagates. An important part of aeroacoustics is the accounting of acoustic noise sources generated by the flow itself, in simple terms, better known as aero-induced acoustic sources. Some works have been devoted to such sources as Bailly and Juvé [27, 28] in which a stochastic noise generation process is solved using linearized Euler equation.

The approach used in the paper is well suited for 3D aeroacoustic simulations in the framework of rapidly evolving design projects. The simulations, which must be carried out for a very large number of flight configurations for each design loop, must therefore be fast but robust with respect to model uncertainties. Within this severe constraint, the use of a more sophisticated CFD is not really adapted. Consequently, the CFD is performed with Euler equations for potential flows and acoustics with the convected Helmholtz equations. These two parts of the aeroacoustic model are well suited for acoustic performance of nacelle liners. Many flight configurations have to be studied in terms of Mach, incidence, and environment. A very large number of 3D simulations must be performed for optimizing the design and certification aspects. Finally, the acoustic source terms are neglected as the fan acoustic excitation is accounted for under the form of a duct modal expansion.

In the framework of nacelle intake liners performance prediction, the following axes can be considered.

1) Noise generation, propagation, and transmission in a moving fluid. In order to understand the mechanisms responsible for the noise production, some studies have been carried out such as [22, 29,-31] on fan noise propagation in duct, and [23] on the transmission/radiation of sound and vibration. This axis is of major importance since knowing the phenomena and mechanisms responsible for acoustic noise in the nacelle allows for developing solutions that are more efficient in mitigating it. In the framework of this paper, the noise generation mainly concerns the study of the interaction between the moving fluid and moving mechanical parts such as the fan or the engine internal systems. The propagation of the noise in a ducted environment for which there is an interaction between the flow and the wall (under the form of a boundary layer) is of major importance when studying lined environments. Finally, the transmission/radiation phenomenon can be studied separately and are not performed in the framework of this paper.

2) Acoustic treatments as mechanical systems. A second axis is related to the mechanical system that constitutes the liner itself. Such a mechanical system is an assembly of several components for which the dissipation phenomena are to be well-modeled so it can be designed to be optimal at specific frequencies (tunable to specific frequencies). The main quantity used for characterizing a liner is its acoustic impedance. The theory of impedance calculation has been introduced by Guess [32] for the mathematical formulation of perforations, or Parrott, Jones, and Watson [2, 33, 34] for the assembly of several parts and the effects of liner geometry on its acoustic impedance. In presence of a moving fluid, the dissipation phenomena are more complex and have been studied by several authors, especially at NASA and ONERA, using experiments ([3, 35,-39]), and/or simulations, such as CFD [40-46] or computational aeroacoustic codes (CAA) [21, 47,-49].

More precisely, in order to account for lined surfaces in presence of a grazing flow, the impedance of liners is calculated using models that are adapted to their physical properties. The liners studied in the present paper are single degree of freedom liners (SDOF) for which the resistive facesheet can either be a simple perforated sheet or topped with a wiremesh surface. In order to characterize perforated plates, the Guess model [32], widely used in the literature, is also used in the present work. This model allows for accounting for the geometry of the sheet in terms of percentage of open area (or porosity), sheet thickness, and perforation diameter. Concerning the wiremesh facesheet, the model used is derived from Airbus expertise, based on the work of several authors [50-53]. This model takes as input parameters the DC flow resistances of the sheet measured at different flow velocities $\left(R_{20}, R_{150}\right.$, see Section [X] , the nonlinear 
factor, and the percentage of open area of the perforated sheet on which the wiremesh is installed. Once the facesheet impedance is described, its transfer matrix between upper and lower surfaces is written. The same method is done for the honeycomb core, and the backing skin. Finally, the product of all transfer matrices gives the relation between all parts of a Single degree of freedom assembly (in our case), or multiple degree of freedom assemblies, as demonstrated by Parrott and Jones in [33]. For page limitation reasons, the detailed modeling of the liner used in this work cannot be presented here. However the reader can find a complete description in Appendix C of [54].

3) Acoustic absorption using liners, and liner performance. A final axis concerns the absorption of acoustic perturbation in lined ducts in presence of a moving fluid. This axis is devoted to the optimization of such liners in their operating environment in order to maximize their ability to absorb the noise produced by the fan. Studies have been carried out on optimization methods [6, 55-57] in order to improve their effectiveness in finding the combination of design parameters that is the most adequate to tackle a specific noise frequency range. These improvements concern the computational tools used, the design parameters that are the most impacting, and the choice of representation for the fan acoustic excitation. In [55], the interest of an axially-segmented liner instead of a uniform one is demonstrated, using a multi-modal representation of the fan noise, as it is done in the present work. Note that a recent work on an efficient design optimization of the liners in a deterministic framework (without uncertainties) can be found in [58].

\section{B. Summarizing the methodology for constructing the nominal computational model}

First, we will present the BVP associated with 3D inner, outer, and duct domains of the intake acoustic radiation problem. The linear convected Helmholtz equation [10, 12,-14, 24] is used in the inner domain (near-field) and in the outer domain (far-field), involving the velocity and mass density fields of the flow computed using CFD. The boundary condition on the liner is the Myers condition [31, 59, 60] involving the local admittance of the liner model [32] depending on the spatial point and on the frequency. At infinity, the outward Sommerfeld radiation condition is written. For the fan excitation (see for instance [30] and [15]), the acoustic velocity field is represented on the family of acoustic duct modes and allows for ensuring the coupling between the semi-infinite duct and the inner domain on the fan boundary, and for representing the acoustic excitation produced by the fan. It should be noted that the duct wall is rigid and its cross-section is assumed to be constant. Inside the duct, the flow is assumed to be uniform. Consequently, the duct modes are explicitly known.

Then, the finite/infinite element discretization of the weak formulation of the BVP is performed, which allows for obtaining the computational model in the frequency domain. The unknowns of this computational model are the discretized acoustic velocity potential fields in the inner domain and in the outer domain, and the vector of the coefficients describing the unknown reflected sound field. In this computational model, the acoustic source term depends on the vector of the known coefficients of the incident sound field describing the fan acoustic excitation.

Finally, we introduce the frequency-dependent computational model that is a very large linear complex matrix system, which induces a large computational cost when exploring the frequency band of analysis. In addition, the nonparametric probabilistic approach of uncertainties (see [7, 61] and also [1]) has to be implemented in the computational model. For these two reasons, a computational reduced-order model depending on the frequency is constructed. For this purpose, two ingredients are introduced [9, 57]. The first one is a frequency-by-frequency static condensation of the aeroacoustic dofs on the liner dofs. Since the number of liner dofs is generally large, the reduced-order model is constructed by introducing a pellicular basis constituted of a set of acoustic modes in a pellicular domain that is related to liner dofs. All this methodology (deterministic and probabilistic parts) has been implemented in commercial software Actran/TM [9, 62, 63], which will be used for the numerical simulations presented in Section VII

\section{Definition of the geometry of the aeroacoustic problem}

For liner performance assessment, an aeroacoustic modeling of the acoustic radiation problem of a nacelle intake is used. The BVP associated with this model (see Fig. 11and Fig. 2) is formulated using three domains. In Fig. 2, the first domain $\Omega_{\mathrm{i}}$ corresponds to the nacelle near-field, which is discretized by finite elements. The second domain $\Omega_{\mathrm{o}}$ corresponds to the far-field, which is discretized by infinite elements. The third domain $\Omega_{\text {duct }}$ allows for analytically calculating the acoustic excitation imposed by the fan, for which the model is based on analytical duct modes ([30]). In order to numerically solve the intake acoustic radiation problem, the associated BVP must be written involving a propagation equation and boundary conditions. For a given flight condition, the flow computation assumes that the fluid 
is inviscid (viscous and thermal effects are neglected) and is performed by solving the potential Euler equations on an appropriate CFD mesh. From these CFD computations, it can be deduced the steady-flow velocity field and the steady-flow mass density field. The convected acoustic wave equation comes from the derivation of the Navier-Stokes equations ([30], [14]), for which it is assumed an irrotational locally isentropic flow. The linearized convected acoustic equation is written in the frequency domain (assuming that fluctuating thermodynamic quantities are small compared to their mean-flow counterpart).



Fig. 2 2D section of the half-3D geometry for the BVP corresponding to the nacelle scheme in Fig 1.

Fig. 2 is a 2D section of the half-3D geometry of the inlet acoustic problem, in which $\Omega_{\mathrm{i}}$ is the inner bounded open domain of $\mathbb{R}^{3}$ with boundary $\partial \Omega_{\mathrm{i}}=\Gamma_{\mathrm{io}} \cup \Gamma_{\mathrm{h}} \cup \Gamma \cup \Gamma_{\mathrm{f}}$ and where $\Omega_{\mathrm{o}}=\mathbb{R}^{3} \backslash \bar{\Omega}_{\mathrm{i}}$ is the outer unbounded open domain of $\mathbb{R}^{3}$, whose boundary is $\partial \Omega_{0}$. The generic point in $\mathbb{R}^{3}$ is denoted as $\mathbf{r}=(x, y, z)$, for which the direction of coordinate $z$ coincides with the rotation axis of the fan. The uniform inflow is in $z$-direction.

Definition of the geometry and boundary conditions for inner domain $\Omega_{\mathbf{i}}$. The boundary part $\Gamma$ corresponds to the coupling interface between $\Omega_{\mathrm{i}}$ and the liner, while $\Gamma_{\mathrm{f}}$ is the boundary part on which the fan excitation is applied. The inner problem implies the use of the following boundary conditions:

- $\Gamma_{\text {io }}$ (inner outer) represents a nonphysical interface between inner and outer domains, on which the continuity condition is written for the acoustic velocity potential and for its gradient.

- $\Gamma_{\mathrm{h}}$ (hardwall) corresponds to rigid or "hardwall" part on which a zero normal acoustic velocity is applied.

- $\Gamma$ (liner) corresponds to the acoustic treatment characterized by its impedance.

- $\Gamma_{f}(f a n)$ corresponds to the part of the boundary on which act acoustic sources induced by the fan.

Definition of the geometry and boundary conditions for outer domain $\Omega_{\mathbf{0}}$. The boundary $\partial \Omega_{\mathrm{o}}$ of $\Omega_{\mathrm{o}}$ is rewritten as $\Gamma_{\mathrm{io}} \cup \Gamma_{\mathrm{ho}}$ in which $\Gamma_{\mathrm{ho}}=\Gamma_{\mathrm{h}} \cup \Gamma \cup \Gamma_{\mathrm{f}}$ (this means that $\partial \Omega_{\mathrm{o}}=\partial \Omega_{\mathrm{i}}$ ). For the acoustic wave propagation in the outer domain, $\Gamma_{\text {ho }}$ corresponds to a rigid wall on which the normal acoustic velocity will be equal to zero.

The outer problem implies the use of the following boundary conditions:

- $\Gamma_{\text {io }}$ (inner outer) is defined above. Note that infinite boundary elements will be used on $\Gamma_{\text {io }}$ for taking into account the effects of $\Omega_{\mathrm{o}}$ on $\Omega_{\mathrm{i}}$ through the coupling interface $\Gamma_{\mathrm{io}}$.

- $\Gamma_{\text {ho }}$ (hardwall outer) corresponds to a rigid wall characterized by a zero normal acoustic velocity.

Definition of the geometry and boundary conditions for the semi-infinite duct $\Omega_{\text {duct }}$. A semi-infinite cylindrical duct $\Omega_{\text {duct }}$ is connected to the inner domain $\Omega_{\mathrm{i}}$ for which the coupling interface is the fan plane $\Gamma_{f}$. The boundary $\partial \Omega_{\text {duct }}$ of $\Omega_{\text {duct }}$ is written as $\partial \Omega_{\text {duct }}=\Gamma_{f} \cup \Gamma_{\text {duct }}$ in which $\Gamma_{\text {duct }}$ is the cylindrical-surface part of the boundary of $\Omega_{\text {duct }}$. It should be noted that this domain is only introduced for generating the acoustic excitation induced by the fan and consequently, has to be viewed as a "fictional domain" with respect to the formulation of the BVP related to $\overline{\Omega_{\mathrm{i}} \cup \Omega_{\mathrm{o}}}$. This is the reason why $\Omega_{\mathrm{o}}=\mathbb{R}^{3} \backslash \bar{\Omega}_{\mathrm{i}}$. In this semi-infinite duct, the sound field is the sum of a given incident sound field 
and an unknown reflected sound field, which will be represented on the duct modes. The wall of the boundary of $\Omega_{\text {duct }}$ is rigid on which a zero normal acoustic velocity is applied.

\section{Boundary value problem associated with the inner, outer, and duct domains of the intake acoustic radiation problem}

The linear convected Helmholtz equation is used in the inner domain $\Omega_{\mathrm{i}}$ and in the outer domain $\Omega_{\mathrm{o}}$ for which $\psi_{\mathrm{i}}$ and $\psi_{\mathrm{o}}$ are the acoustic velocity potential fields, respectively. The reader can find the developments in Section II-D of Supplementary material for the BVP in the inner domain $\Omega_{\mathrm{i}}$ (near-field), for the BVP in the outer domain $\Omega_{\mathrm{o}}$, and for the BVP in the semi-infinite duct $\Omega_{\text {duct }}$ for the inlet case and duct modes.

Concerning the fan excitation (see for instance [30] and [15]), the acoustic velocity potential field $\varphi$ in $\Omega_{\text {duct }}$ will be represented on the family of acoustic duct modes and will allow (i) for ensuring the coupling between the semi-infinite duct $\Omega_{\text {duct }}$ and the inner domain $\Omega_{\mathrm{i}}$ on boundary $\Gamma_{f}$ (see Fig. 2), and (ii) for representing the acoustic excitation produced by the fan. In this work, it is assumed that $\Omega_{\text {duct }}$ is a straight cylinder with $z$-axis, for which its cross-section is constant and consequently, independent of $z$. The steady flow in $\Omega_{\text {duct }}$ is assumed to be subsonic and uniform along $z$-direction. The acoustic velocity potential field $\varphi$ is written by keeping only a finite number $N_{d}$ of duct modes and by showing the contribution of the given incident sound field (represented by the symbol +) that represents the acoustic excitation induced by the fan and the contribution of the unknown associated reflected sound field (represented by the symbol -). Therefore, function $\varphi(x, y, z)$ is written as

$$
\varphi(x, y, z)=\sum_{\alpha=1}^{N_{d}} \varphi_{\alpha}(x, y)\left(c_{\alpha}^{+} e^{j k_{z \alpha}^{+} z}+c_{\alpha}^{-} e^{j k_{z_{\alpha}}^{-} z}\right),
$$

in which $k_{z_{\alpha}}^{+}$is the axial wavenumber of the wave propagating in the upstream direction and $k_{z_{\alpha}}^{-}$is the axial wavenumber of the wave propagating in the downstream direction, such that $k_{z_{\alpha}}=\left(k M_{\infty} \pm \sqrt{k^{2}-\beta^{2} k_{x y_{\alpha}}^{2}}\right) / \beta^{2}$ with $k_{x y_{\alpha}}$ the transversal wavenumber of a given duct mode $\alpha$. In these equations, $k=\omega / c$ is the acoustic wave number, $M_{\infty}=\left\|\mathbf{v}_{\infty}\right\| / c$ is the Mach number associated with the uniform flow, and $\beta=\sqrt{1-M_{\infty}^{2}}$. It should be noted that the acoustic excitation induced by the fan, which is associated with the incident sound field, is defined by giving the complex vector $\mathbf{c}^{+}=\left(c_{1}^{+}, \ldots, c_{N_{d}}^{+}\right)$, while the unknown reflected sound field depends on the vector $\mathbf{c}^{-}=\left(c_{1}^{-}, \ldots, c_{N_{d}}^{-}\right)$and will be included with all the unknown variables of the acoustic problem. For describing the acoustic excitation of the fan, $\mathbf{c}^{+}$can be chosen as dependent on $\omega$ and will then be denoted as $\mathbf{c}^{+}(\omega)$. On the other hand, whether or not $\mathbf{c}^{+}$depends on $\omega$, as $\mathbf{c}^{-}$is an unknown of the problem, $\mathbf{c}^{-}$always depends on $\omega$, which we will denote by $\mathbf{c}^{-}(\omega)$.

\section{E. Finite/Infinite Element discretization of the weak formulation}

The weak formulation of the BVP with $\varphi$ given by (1) is discretized by the Finite/Infinite Element Method (FEM/IEM). For all $\omega$ fixed in $\mathbb{R}$, let $\psi_{\mathrm{i}}(\omega)$ be the complex vector in $\mathbb{C}^{N_{\mathrm{i}}}$ corresponding to the finite element discretization of field $\psi_{\mathrm{i}}(\omega)$ in which $N_{\mathrm{i}}$ is the number of degrees of freedom (dofs). Let $\psi_{\mathrm{o}}(\omega)$ be the complex vector in $\mathbb{C}^{N_{\mathrm{o}}}$ corresponding to the finite/infinite element discretization of field $\psi_{\mathrm{o}}(\omega)$ in which $N_{\mathrm{o}}$ is the number of dofs. We then obtain the computational model in terms of $\psi_{\mathrm{i}}(\omega), \psi_{\mathrm{o}}(\omega)$, and $\mathbf{c}^{-}(\omega)$, which is written as

$$
\left[\begin{array}{ccc}
{\left[A_{\mathrm{i}}(\omega)\right]+\left[Z_{\mathrm{i}}(\omega)\right]} & {[H(\omega)]} & -\left[\mathcal{E}_{\mathrm{if}}\right]\left[F^{-}(\omega)\right] \\
-[H(\omega)]^{T} & {\left[A_{\mathrm{o}}(\omega)\right]} & {[0]} \\
{[E]} & {[0]} & -\left[R^{-}(\omega)\right]
\end{array}\right]\left[\begin{array}{c}
\boldsymbol{\psi}_{\mathrm{i}}(\omega) \\
\boldsymbol{\psi}_{\mathrm{o}}(\omega) \\
\mathbf{c}^{-}(\omega)
\end{array}\right]=\left[\begin{array}{c}
{\left[\mathcal{E}_{\mathrm{if}}\right]\left[F^{+}(\omega)\right]} \\
{[0]} \\
{\left[R^{+}(\omega)\right]}
\end{array}\right] \mathbf{c}^{+}(\omega),
$$

in which,

- the $\left(N_{\mathrm{i}} \times N_{\mathrm{i}}\right)$ complex matrix $\left[A_{\mathrm{i}}(\omega)\right]$, the $\left(N_{\mathrm{o}} \times N_{\mathrm{o}}\right)$ complex matrix $\left[A_{\mathrm{o}}(\omega)\right]$, and the $\left(N_{\mathrm{i}} \times N_{\mathrm{o}}\right)$ complex matrix $[H(\omega)]$ correspond to the block decomposition of the aeroacoustic impedance matrix.

- the diagonal $\left(N_{\mathrm{d}} \times N_{\mathrm{d}}\right)$ complex matrices $\left[R^{+}(\omega)\right]$ and $\left[R^{-}(\omega)\right]$ correspond to the incident and to the reflected modal participations in the duct domain $\Omega_{\text {duct }}$.

- the $\left(N_{\mathrm{d}} \times N_{\mathrm{i}}\right)$ constant real matrix [E] corresponds to the finite element discretization of the inner product in the space of all the square integrable functions on boundary $\Gamma_{\mathrm{f}}$.

- the $\left(N_{\mathrm{i}} \times N_{\mathrm{f}}\right)$ real matrix [ $\left.\mathcal{E}_{\mathrm{if}}\right]$ constituted of ones and zeros, allows for selecting the dofs related to boundary $\Gamma_{\mathrm{f}}$.

- the $\left(N_{\mathrm{f}} \times N_{\mathrm{d}}\right)$ complex matrices $\left[F^{+}(\omega)\right]$ and $\left[F^{-}(\omega)\right]$ represent the discretization of the incident and the reflected modal participations in the inner domain $\Omega_{\mathrm{i}}$. 


\section{Computational reduced-order model}

\section{A. Frequency-by-frequency static condensation with respect to the liner dofs and expression of the fan acoustic excitation}

In Section IV] we will present the probabilistic modeling of uncertainties in the computational model defined by Eq. (2). These uncertainties will be taken into account for both the parametric uncertainties and the model uncertainties induced by the modeling errors in the aeroacoustic and liner computational models. Consequently, we need to reorganize the algebraic structure of the complex matrix equation defined by Eq. (2) in order to exhibit the complex vector $\psi_{\ell}(\omega)$ belonging to $\mathbb{C}^{N_{\ell}}$ of the $N_{\ell}$ dofs of the liner and the complex vector $\psi_{a}$ belonging to $\mathbb{C}^{N_{a}}$ of the $N_{a}$ other dofs of $\left(\psi_{\mathrm{i}}(\omega), \psi_{\mathrm{o}}(\omega), \mathbf{c}^{-}(\omega)\right)$. This means that $N_{a}=N_{\mathrm{i}}+N_{\mathrm{o}}+N_{d}-N_{\ell}$ and that,

$$
\left[\begin{array}{l}
\psi_{\mathrm{i}}(\omega) \\
\psi_{\mathrm{o}}(\omega) \\
\mathbf{c}^{-}(\omega)
\end{array}\right] \text { is rewritten as }\left[\begin{array}{l}
\boldsymbol{\psi}_{\ell}(\omega) \\
\boldsymbol{\psi}_{a}(\omega)
\end{array}\right], \quad N_{\mathrm{i}}+N_{\mathrm{o}}+N_{d}=N_{\ell}+N_{a} .
$$

Since $\Gamma \cap \Gamma_{\mathrm{f}}=\{\emptyset\}$, we have

$$
\left[\begin{array}{c}
{\left[\mathcal{E}_{\mathrm{if}}\right]\left[F^{+}(\omega)\right]} \\
{[0]} \\
{\left[R^{+}(\omega)\right]}
\end{array}\right] \mathbf{c}^{+}(\omega) \text { is rewritten as }\left[\begin{array}{c}
\mathbf{0} \\
\mathbf{f}_{a}(\omega)
\end{array}\right] \quad, \quad N_{\mathrm{i}}+N_{\mathrm{o}}+N_{d}=N_{\ell}+N_{a},
$$

in which the $\mathbb{C}^{N_{a}}$-vector $\mathbf{f}_{a}(\omega)$ can be written as

$$
\mathbf{f}_{a}(\omega)=\left[\mathcal{B}_{a}(\omega)\right] \mathbf{c}^{+}(\omega),
$$

where $\left[\mathcal{B}_{a}(\omega)\right]$ is a known complex $\left(N_{a} \times N_{d}\right)$ matrix. Using Eqs. (3) and (4), the computational model defined by Eq. (2) can be rewritten in a block form as

$$
\left[\begin{array}{ll}
{\left[A_{\ell \ell}(\omega)\right]} & {\left[A_{\ell a}(\omega)\right]} \\
{\left[A_{a \ell}(\omega)\right]} & {\left[A_{a a}(\omega)\right]}
\end{array}\right]\left[\begin{array}{l}
\boldsymbol{\psi}_{\ell}(\omega) \\
\boldsymbol{\psi}_{a}(\omega)
\end{array}\right]+\left[\begin{array}{cc}
{\left[Z_{\ell}(\omega)\right]} & 0 \\
0 & 0
\end{array}\right]\left[\begin{array}{l}
\boldsymbol{\psi}_{\ell}(\omega) \\
\boldsymbol{\psi}_{a}(\omega)
\end{array}\right]=\left[\begin{array}{c}
\mathbf{0} \\
\mathbf{f}_{a}(\omega)
\end{array}\right]
$$

For solving Eq. (6), the following $\omega$-by- $\omega$ static condensation (Schur complement) is performed. It consists in eliminating the vector $\mathbf{x}_{a}$ in the following matrix equation,

$$
\left[\begin{array}{ll}
{\left[A_{\ell \ell}(\omega)\right]} & {\left[A_{\ell a}(\omega)\right]} \\
{\left[A_{a \ell}(\omega)\right]} & {\left[A_{a a}(\omega)\right]}
\end{array}\right]\left[\begin{array}{l}
\mathbf{x}_{\ell} \\
\mathbf{x}_{a}
\end{array}\right]=\left[\begin{array}{c}
\mathbf{0} \\
\mathbf{f}_{a}(\omega)
\end{array}\right] .
$$

This elimination yields $\left[\mathcal{A}_{\ell}(\omega)\right] \mathbf{x}_{\ell}=\mathcal{F}_{\ell}(\omega)$, in which the $\left(N_{\ell} \times N_{\ell}\right)$ complex matrix $\left[\mathcal{A}_{\ell}(\omega)\right]$ is defined by

$$
\left[\mathcal{A}_{\ell}(\omega)\right]=\left[A_{\ell \ell}(\omega)\right]-\left[A_{\ell a}(\omega)\right]\left[A_{a a}(\omega)\right]^{-1}\left[A_{a \ell}(\omega)\right] .
$$

The $N_{\ell}$ complex vector $\mathcal{F}_{\ell}(\omega)$ is written as $\mathcal{F}_{\ell}(\omega)=-\left[A_{\ell a}(\omega)\right]\left[A_{a a}(\omega)\right]^{-1} \mathbf{f}_{a}(\omega)$, which can be rewritten, using Eq. (5), as $\mathcal{F}_{\ell}(\omega)=\left[\mathcal{B}_{\ell}(\omega)\right] \mathbf{c}^{+}(\omega)$ in which the $\left(N_{\ell} \times N_{d}\right)$ complex matrix $\left[\mathcal{B}_{\ell}(\omega)\right]$ is defined by

$$
\left[\mathcal{B}_{\ell}(\omega)\right]=-\left[A_{\ell a}(\omega)\right]\left[A_{a a}(\omega)\right]^{-1}\left[\mathcal{B}_{a}(\omega)\right] .
$$

Using this $\omega$-by- $\omega$ static condensation, the elimination of $\psi_{a}(\omega)$ in Eq. (6) yields,

$$
\left(\left[\mathcal{A}_{\ell}(\omega)\right]+\left[Z_{\ell}(\omega)\right]\right) \psi_{\ell}(\omega)=\left[\mathcal{B}_{\ell}(\omega)\right] \mathbf{c}^{+}(\omega) .
$$

\section{B. Computational reduced-order model}

Following the explanations given at the beginning of Section III.A the uncertainties will be taken into account by using the nonparametric probabilistic approach [7]. Such an approach requires the introduction of a reduced-order model associated with Eq (10). For such a construction we need to introduce a reduced-order basis that is chosen as a pellicular basis (see [9, 64]) whose construction is summarized in Section III-B of Supplementary material. Let $n_{\ell}<N_{\ell}$ be the number of the pellicular basis vectors represented by the $\left(N_{\ell} \times n_{\ell}\right)$ real matrix $[\Phi]$. The computational reduced-order model is obtained by projecting Eq. 10 on the subspace spanned by $[\Phi]$ and is thus written as 


$$
\begin{gathered}
\psi_{\ell}(\omega)=[\Phi] \mathbf{q}(\omega), \\
([\mathbb{A}(\omega)]+[\mathbb{Z}(\omega)]) \mathbf{q}(\omega)=[\mathbb{B}(\omega)] \mathbf{c}^{+}(\omega),
\end{gathered}
$$

in which $\mathbf{q}(\omega)$, which belongs to $\mathbb{C}^{n_{\ell}}$, is a complex vector of the generalized coordinates, and where the $\left(n_{\ell} \times n_{\ell}\right)$ complex matrix $[\mathbb{A}(\omega)]$, the $\left(n_{\ell} \times n_{\ell}\right)$ complex matrix $[\mathbb{Z}(\omega)]$, and the $\left(n_{\ell} \times N_{d}\right)$ complex matrix $[\mathbb{B}(\omega)]$ are such that

$$
\begin{aligned}
{[\mathbb{A}(\omega)] } & =[\Phi]^{T}\left[\mathcal{A}_{\ell}(\omega)\right][\Phi], \\
{[\mathbb{Z}(\omega)] } & =[\Phi]^{T}\left[Z_{\ell}(\omega)\right][\Phi], \\
{[\mathbb{B}(\omega)] } & =[\Phi]^{T}\left[\mathcal{B}_{\ell}(\omega)\right] .
\end{aligned}
$$

Once $\psi_{\ell}(\omega)$ is known, $\psi_{a}(\omega)$ is computed by using the second line of Eq. (6) in which $\mathbf{f}_{a}(\omega)$ is substituted by its expression given by Eq. [5],

$$
\left[A_{a a}(\omega)\right] \psi_{a}(\omega)=\left[\mathcal{B}_{a}(\omega)\right] \mathbf{c}^{+}(\omega)-\left[A_{a \ell}(\omega)\right] \psi_{\ell}(\omega) .
$$

We introduce a vector $\mathbf{q o I}(\omega)$ of quantities of interest related to the far-field acoustic pressure at several given spatial points. This vector can always be written as

$$
\mathbf{q o I}(\omega)=\left[\bigcirc_{\mathrm{obs}}(\omega)\right] \psi_{a}(\omega),
$$

in which $\left[\mathrm{O}_{\mathrm{obs}}(\omega)\right]$ is a given observation matrix. Combining Eqs. (11) and (16) shows that Eq. (17) can be rewritten as

$$
\operatorname{qoI}(\omega)=\left[\mathbb{F}_{\mathrm{qoI}}(\omega)\right] \mathbf{c}^{+}(\omega)-\left[\mathbb{A}_{\mathrm{qoI}}(\omega)\right] \mathbf{q}(\omega),
$$

in which the matrices $\left[\mathbb{F}_{\text {qoI }}(\omega)\right]$ and $\left[\mathbb{A}_{\mathrm{qoI}}(\omega)\right]$ are formally given by $\left[\mathbb{F}_{\mathrm{qoI}}(\omega)\right]=\left[\mathcal{O}_{\mathrm{obs}}(\omega)\right]\left[A_{a a}(\omega)\right]^{-1}\left[\mathcal{B}_{a}(\omega)\right]$ and $\left[\mathbb{A}_{\mathrm{qoI}}(\omega)\right]=\left[\bigcirc_{\mathrm{obs}}(\omega)\right]\left[A_{a a}(\omega)\right]^{-1}\left[A_{a \ell}(\omega)\right][\Phi]$.

In the framework of the nonparametric probabilistic approach, the uncertainties in the computational model are implemented by substituting complex matrices $[\mathbb{A}(\omega)]$ and $[\mathbb{Z}(\omega)]$ by random complex matrices $[\mathbf{A}(\omega)]$ and $[\mathbf{Z}(\omega)]$ in Eq. (12). The uncertainties on vector $\mathbf{c}^{+}(\omega)$ are taken into account by the parametric probabilistic, which consists in modeling $\mathbf{c}^{+}(\omega)$ by a random vector $\mathbf{C}^{+}(\omega)$.

\section{Probabilistic model for uncertainty quantification}

As previously explained, three sources of uncertainties are taken into account. The first one is related to uncertainties induced by modeling errors in the impedance matrix of the liner appearing in the computational reduced-order model. The second one corresponds to model uncertainties in the aeroacoustic part of the computational model including the mean-flow computation (CFD), the acoustic equations in moving fluids, and the duct acoustic related to the fan excitation. The third one is related to uncertainties in the acoustic excitation induced by the fan. The stochastic solver of the random equation is presented. For each value of the frequency, the Stochastic Reduced-Order Model (SROM) is solved by the Monte Carlo numerical simulation method [65]. The convergence analysis is carried out with respect to the number of realizations. For the first two sources of uncertainties, the nonparametric probabilistic approach is used, in particular the construction of the probabilistic model of the random matrices (see [7]). For the third one, the parametric probabilistic approach is used.

\section{A. Brief summary of the nonparametric probabilistic approach of uncertainties}

For a given flight condition and given environment, the BVP considered is that of aeroacoustics whose equations are those of the linearized convected acoustic wave equations formulated in the frequency domain. This aeroacoustic boundary value problem depends on physical system parameters (geometry of the very complex and unbounded domain, velocity field of the flow that is calculated by the CFD for a given flight condition, mechanical and acoustic parameters of the liner model) but also depends on modeling aspects (acoustic excitation induced by the fan, use of the linearized convected Helmholtz equation instead of the Navier-Stokes equations, boundary conditions related to the impedance of the liner). The development of the aeroacoustic computational model for this BVP requires the introduction of simplifications and approximations: simplification of the geometry, simplification of the model describing the acoustic excitation of the fan, 
simplification of the internal geometry of the reactor, introduction of two vector bases for constructing the reduced-order computational model, and simplification of the elastoacoustic model used for building the impedance of the liner. In this aeroacoustic problem, there are therefore many model uncertainties induced by modeling errors. It is known for a long time that model uncertainties cannot be taken into account by parametric probabilistic approaches. It is for this reason that the nonparametric probabilistic approach of model uncertainties was proposed in 2000 [61, 66, 67] and has given rise to numerous developments and validation works over the past 20 years. This approach has now become a standard method, which is, for example, implemented in commercial software such as MSC Nastran software.

Concepts, mathematical developments, and experimental validations can be found in [7, 68, 69] and also in [7, 64, 70, 71] for elastoacoustic problems, and for more recent advances, for instance, in [72, 73]. Because of model uncertainties induced by modeling errors, the BVP that is constructed, from which the computational model is derived, does not correspond to the physical reality that is modeled. It is therefore necessary to generate a stochastic family of boundary value problems whose solution space is large enough to contain the targets such as experimental results. We recall that the family generated by a parametric approach does not allow the construction of such a family (see for example [7]). The so-called nonparametric probabilistic approach consists in generating this stochastic family by modeling operators of the boundary problems by random operators whose levels of statistical fluctuations, that is to say, the levels of uncertainty, are controlled by hyperparameters. The mathematical properties of the considered operators are preserved during the construction of the associated random operators. One thus generates a family of stochastic solutions, which obviously do not satisfy the initial BVP by construction. If all the mathematical concepts and developments associated with the nonparametric probabilistic approach can be used for the aeroacoustic problem considered in this work, it is necessary to specify the algebraic properties of each random operator considered in order to use the appropriate subsets of random matrices and to build their probabilistic models. It should be noted that two types of nonparametric probabilistic approach can be used to model matrix-valued random impedances. The first allows causality to be taken into account explicitly, as proposed in [74] and in [7, 75, 76]. However, the implementation of this type of model is relatively complex and generates significant calculation costs for large dimensions (which is the case here). We have therefore preferred to use an approach a little less refined but simpler to implement and not generating significant numerical costs. These are the developments that are presented in this section.

\section{B. Construction of the probabilistic model of the reduced liner impedance matrix}

This construction of the probabilistic model of the random liner matrix $[\mathbf{Z}(\omega)]$ is given in Section IV-B of Supplementary material using the algebraic properties of the liner impedance complex matrix $[\mathbb{Z}(\omega)]$, which are presented in Section III-C of Supplementary material. The probability distribution of random matrix $[\mathbf{Z}(\omega)]$ depends on a frequency-independent hyperparameter $\delta_{Z}>0$, which allows the level of uncertainties of the liner impedance to be controlled. It should be noted that hyperparameter $\delta_{Z}$ allows for quantifying the global level of uncertainties for matrix-valued random impedance $\left[\mathbf{Z}_{i}(\omega)\right]$ that depends on two modeling errors related to the Myers condition and to the local admittance of the liner. These two modeling errors cannot be separated because the impedance operator cannot be separated in two algebraically independent operators, as it can be seen in its weak formulation before finite element discretization. Consequently, the two sources of modeling errors cannot be separated in the nonparametric model and therefore, there is only one hyperparameter.

\section{Construction of the probabilistic model of the reduced aeroacoustic matrix}

Similarly to the liner impedance matrix, the construction of the probabilistic model of the random aeroacoustic matrix $[\mathbf{A}(\omega)]$ is given in Section IV-C of Supplementary material, using the algebraic properties of the aeroacoustic impedance complex matrix $[\mathbb{A}(\omega)]$, which are given in Section III-D of Supplementary material. The probability distribution of random matrix $[\mathbf{A}(\omega)]$ depends on a frequency-independent hyperparameter $\delta_{A}>0$, which allows for controlling the level of uncertainties of the aeroacoustic impedance.

\section{Parametric probabilistic modeling of the fan acoustic excitation}

There are two possible approaches for constructing the parametric probabilistic model of the fan excitation. The first one consists in directly considering the modal components $\left\{c_{\alpha}^{+}(\omega), \alpha=1, \ldots, N_{d}\right\}$ of the duct modes, while the second one consists in describing the probabilistic model for the modal intensity $\left\{I_{\alpha}^{+}(\omega), \alpha=1, \ldots, N_{d}\right\}$ and using the expression of $c_{\alpha}^{+}(\omega)$ as a function of $I_{\alpha}^{+}(\omega)$. In the present paper, we only present the latter. In addition, the reader will find the relation between $c_{\alpha}^{+}(\omega)$ and $I_{\alpha}^{+}(\omega)$ in Appendix B of the Dangla thesis [54] (this relation is not given because the required 
explanations are long enough and relatively fastidious).

Probabilistic model of $I_{\alpha}^{+}(\omega)$. For civil aircraft applications, a deterministic mean model, $\underline{I}_{\alpha}^{+}(\omega)$, of the modal intensity is often used by the manufacturers and this mean model has to be accounted for in the construction of the probabilistic model of $I_{\alpha}^{+}(\omega)$. Consequently, $\underline{I}_{\alpha}^{+}(\omega)$ is chosen as the statistical mean value of the random variable $I_{\alpha}^{+}(\omega)$. For the construction of the probability distribution of the modal intensity, the available information are the following: $I_{\alpha}^{+}(\omega)$ is a positive-valued random variable, its mean value is $\underline{I}_{\alpha}^{+}(\omega)$, and the support of its probability distribution is the given positive interval $\mathcal{J}_{\alpha}(\omega)=\left[\underline{I}_{\alpha}^{+}(\omega)\left(1-\sigma_{\alpha}(\omega)\right), \underline{I}_{\alpha}^{+}(\omega)\left(1+\sigma_{\alpha}(\omega)\right)\right] \subset \mathbb{R}^{+}$depending on $\alpha$ and on a positive hyperparameter $0<\sigma_{\alpha}(\omega)<1$. It should be noted that no information is assumed to be available concerning the statistical dependence of the random variables $I_{1}^{+}(\omega), \ldots, I_{N_{d}}^{+}(\omega)$. Using the MaxEnt principle of Information Theory, it is concluded that the random variables $I_{1}^{+}(\omega), \ldots, I_{N_{d}}^{+}(\omega)$ are statistically independent and the probability distribution of each $I_{\alpha}^{+}(\omega)$ is uniform with support $\mathcal{J}_{\alpha}(\omega)$ and with mean value $\underline{I}_{\alpha}^{+}(\omega)$. Therefore, the random variable $I_{\alpha}^{+}(\omega)$ can be written as

$$
I_{\alpha}^{+}(\omega)=\underline{I}_{\alpha}^{+}(\omega)\left(1+\sigma_{\alpha}(\omega) U_{\alpha}(\omega)\right) \quad, \quad 0<\sigma_{\alpha}(\omega)<1,
$$

in which $U_{\alpha}(\omega)$ is a centered uniform random variable on the interval $[-1,1]$.

\section{E. Construction of the SROM by using the nonparametric probabilistic approach of uncertainties}

As previously explained, the nonparametric probabilistic approach of both the parametric uncertainties and the model uncertainties induced by modeling errors in the computational model of the acoustic radiation of an inlet consists in modeling matrix $[\mathbb{Z}(\omega)]$ defined by Eq. [14 by the random complex matrix $[\mathbf{Z}(\omega)]$ and $[\mathbb{A}(\omega)]$ defined by Eq. 13 , by the random complex matrix $[\mathbf{A}(\omega)]$. The introduction of uncertainties on the fan acoustic excitation consists in modeling $\mathbf{c}^{+}(\omega)=\left(c_{1}^{+}(\omega), \ldots, c_{N_{d}}^{+}(\omega)\right)$ by the random complex vector $\mathbf{C}^{+}(\omega)=\left(C_{1}^{+}(\omega), \ldots, C_{N_{d}}^{+}(\omega)\right)$ that is expressed as a function of $\mathbf{I}^{+}(\omega)=\left(I_{1}^{+}(\omega), \ldots, I_{N_{d}}^{+}(\omega)\right)$ (see Section IV.D. From Eqs. (18) and [12), it can be deduced that the random vector $\mathbf{Q o I}(\omega)$ depending on $\omega$ is such that

$$
\mathbf{Q o I}(\omega)=\left[\mathbb{F}_{\mathrm{qoI}}(\omega)\right] \mathbf{C}^{+}(\omega)-\left[\mathbb{A}_{\mathrm{qoI}}(\omega)\right] \mathbf{Q}(\omega),
$$

in which the random vector $\mathbf{Q}(\omega)$ is such that

$$
([\mathbf{A}(\omega)]+[\mathbf{Z}(\omega)]) \mathbf{Q}(\omega)=[\mathbb{B}(\omega)] \mathbf{C}^{+}(\omega) .
$$

\section{F. Solving the stochastic matrix equation and constructing the confidence regions}

The frequency band of analysis $\left[\omega_{\min }, \omega_{\max }\right]$ is sampled in $n_{\omega}$ points $\left\{\omega_{1}, \ldots \omega_{n_{\omega}}\right\}$. For each $\omega=\omega_{j}$, the Monte Carlo method is used for solving the SROM. It consists in computing a set of $v_{s}$ independent realizations, with $v_{s}$ sufficiently large, of the random quantities of interest by using Eqs. 20) and 21]. From this set of independent realizations, estimates of the statistics for the quantities of interest are computed, such as probability density functions, moments (means, standard deviations), confidence regions. The convergence of the estimates are analyzed with respect to $v_{s}$.

\section{Configuration and computational model of a generic industrial nacelle}

In this section, we present the computational model that is used to assess the performance of an acoustic liner in its operating environment. In a first step, for a given flight condition, CFD is used for calculating the velocity field and the mass density field of the steady flow using an Airbus in-house CFD code based on the potential Euler equations. The reader will be able to find figures of the mass density and velocity fields for the steady flow in Chapter 3 of [54]. In a second step, the computational aeroacoustic model is constructed.

For the computational aeroacoustic model including the liners, the finite/infinite element discretization of the half-3D generic nacelle intake is shown in Fig. 3. The left figure shows the following three fluid boundaries: the liner surfaces $\Gamma$ on which there are two liners: the "Inlet liner" and the "Forward Fan Case (FFC) liner", the fan excitation surface $\Gamma_{f}$ on which the incident acoustic pressure field is imposed through the duct, and the near/far-field surface $\Gamma_{\text {io }}$ on which is imposed the continuity of the acoustic velocity potential field between the near-field and the far-field. The right figure shows the 2D section of the half-3D generic aeroacoustic mesh shown on the left figure. Note that the existence of a system symmetry with a non-symmetric excitation allows for using a half model considering two calculations: 
one for the symmetric part of the excitation (positive excitation azimuthal orders) for which a zero pressure gradient normal to the symmetry plane is imposed and one for the anti-symmetric part (negative excitation azimuthal orders) for which a zero pressure condition is imposed on the symmetry plane. The mesh used for the computation depends on the maximum of the considered frequency. Two mesh cases are considered corresponding to a maximum frequency of $1000 \mathrm{~Hz}$ and $2000 \mathrm{~Hz}$. As it can be seen in Table 1 , there are 458521 nodes in the computational aeroacoustic mesh of the half system for $1000 \mathrm{~Hz}$, and 3056476 nodes for $2000 \mathrm{~Hz}$


Fig. 3 Left: $\Gamma$ (red and orange), $\Gamma_{f}$ (green), and $\Gamma_{\mathrm{io}}$ (purple). Right: 2D section of the 3D aeroacoustic mesh.

\begin{tabular}{c||cccccc|c}
\hline Mesh case & $\begin{array}{c}\text { FEM } \\
\text { of } \Omega_{\mathrm{i}}\end{array}$ & $\begin{array}{c}\text { IEM } \\
\text { of } \Gamma_{\mathrm{i}}\end{array}$ & $\begin{array}{c}\text { Inlet } \\
\text { liner } \Gamma\end{array}$ & $\begin{array}{c}\text { FFC } \\
\text { liner } \Gamma\end{array}$ & $\begin{array}{c}\text { Fan } \\
\text { excitation } \Gamma_{f}\end{array}$ & $\begin{array}{c}\text { Sym } \\
\text { plane }\end{array}$ & $\begin{array}{c}\text { Total } \\
\text { of nodes }\end{array}$ \\
\hline $1000 \mathrm{~Hz}$ & 423498 & 9182 & 4821 & 981 & 3285 & 16559 & 458521 \\
$2000 \mathrm{~Hz}$ & 2939198 & 24805 & 8665 & 1875 & 8277 & 73189 & 3056476 \\
\hline
\end{tabular}

Table 1 Number of mesh nodes of the computational aeroacoustic model for the two mesh cases depending on the maximum frequency.

The quantities of interest are the far-field acoustic pressure that are captured by a "far-field microphone array" located at $46 \mathrm{~m}$ of the nacelle axis (see Fig. (4) and the ring acoustic pressure that are captured by a "ring microphone array" located on the nacelle intake lip. The fan acoustic contributions are observed and allow for computing both the far-field and the ring directivity modal SPL (Sound Pressure Level) quantities. The SPL broadband azimuthal quantity (far-field) consists in calculating the quadratic mean of the acoustic pressure over the cut-on excitation modes, accounting for statistically independent random phases on $[0,2 \pi]$. Whereas, for the ring, azimuthal (or circumferential) SPL are computed using the phase of each microphone and the modal contribution of the fan excitation acquired on each microphone. These quantities of interest are developed in the following section. In the present work, one flight condition is studied, corresponding to the flyover acoustic certification point following ICAO rules [77].

\section{Simulated and experimental quantities of interest}

The experimental environment is a static ground test. The engine is hanged by a mechanical arm, in free space (exterior). Microphones are placed at the intake lip for the modal detection of the acoustic excitation induced by the fan, and microphones are placed in far-field for the acoustic certification of the engine. All the physical reflections, including the reflections on the ground, are taken into account in the post-processing of the measurements and in the computational aeroacoustic model. 



Fig. 4 Left: far-field microphone array. Right: ring microphone array located in the nacelle intake lip.

\section{A. Quantities of interest related to the ring microphone array}

The ring acoustic pressures are written as



in which $N_{\text {mics,r }}=100$ is the number of flush-mounted microphones distributed on the ring (intake lip), $\omega$ is the angular frequency, $m$ is the azimuthal order, $a_{m}(\omega)$ is the complex amplitude of the azimuthal order $m$, and $\eta_{i}=2 \pi(i-1) / N_{\text {mics,r }}$ is the angle in radian of the $i^{\text {th }}$ microphone. Eq. 22) can be rewritten as

$$
\mathbf{p}_{\text {ring }}(\omega)=[B] \mathbf{a}(\omega),
$$

in which $\mathbf{p}_{\text {ring }}(\omega)=\left(p_{\text {ring, }, 1}(\omega), \ldots, p_{\text {ring, } \mathrm{N}_{\text {mics }, \mathrm{r}}}(\omega)\right)$ is a complex vector, where $\mathbf{a}(\omega)=\left(a_{-N_{\text {azi }}}(\omega), \ldots, a_{0}(\omega), \ldots, a_{N_{\text {azi }}}(\omega)\right)$ is a complex vector of amplitudes, and where $[B]$ is the $\left(N_{\text {mics, } \mathrm{r}} \times\left(2 N_{\mathrm{azi}}+1\right)\right)$ complex matrix whose entries are $[B]_{i m}=e^{j m \eta_{i}}$. Assuming that $2 N_{\mathrm{azi}}+1 \leq N_{\text {mics }, \mathrm{r}}$, the azimuthal amplitude of the ring acoustic pressure is represented by the complex amplitude vector $\mathbf{a}(\omega)$ that is constructed by using the left pseudo-inversion of Eq. 23,

$$
\mathbf{a}(\omega)=\left(\left([B]^{*}[B]\right)^{-1}[B]^{*}\right) \mathbf{p}_{\text {ring }}(\omega) .
$$

The azimuthal Sound Pressure Level (SPL) of azimuthal order $m$ of the ring acoustic pressure, expressed in $\mathrm{dB}$, is defined by

$$
\operatorname{SPL}_{\text {ring, } m}(\omega)=10 \log _{10}\left(\frac{\left|a_{m}(\omega)\right|^{2}}{p_{\text {ref }}^{2}}\right) \quad, \quad m \in\left\{-N_{\mathrm{azi}}, \ldots, 0, \ldots, N_{\mathrm{azi}}\right\},
$$

in which $p_{\text {ref }}=2 \times 10^{-6}$ is the pressure of reference. The counterpart of $p_{\text {ring }, i}(\omega), a_{m}(\omega)$, and $\operatorname{SPL}_{\text {ring, } m}(\omega)$ for the experimental measurements are denoted by $p_{\text {ring, } i}^{\exp }(\omega), a_{m}^{\exp }(\omega)$, and $\operatorname{SPL}_{\text {ring, } m}^{\exp }(\omega)$.

\section{B. Quantities of interest related to the far-field microphone array}

There are $N_{\text {mics,ff }}=23$ microphones uniformly distributed between $10^{\circ}$ and $120^{\circ}$ in the arc defining the far-field microphone array (see Fig. 47. For the $i^{\text {th }}$ microphone the far-field acoustic pressure is denoted by $p_{\mathrm{ff}, i}(\omega)$. The Sound Pressure Level (SPL) for the far-field acoustic pressure of the $i^{\text {th }}$ microphone is defined by

$$
\operatorname{SPL}_{\mathrm{ff}, i}(\omega)=10 \log _{10}\left(\frac{\left|p_{\mathrm{ff}, i}(\omega)\right|^{2}}{p_{\mathrm{ref}}^{2}}\right) .
$$

The counterpart of $p_{\mathrm{ff}, i}(\omega)$ and $\operatorname{SPL}_{\mathrm{ff}, i}(\omega)$ for the experimental measurements are denoted by $p_{\mathrm{ff}, i}^{\exp }(\omega)$ and $\operatorname{SPL}_{\mathrm{ff}, i}^{\exp }(\omega)$.

\section{Modeling of the mean modal intensity $\underline{I}_{\alpha}^{+}$}

\section{Parameterization of the mean modal density also called modal content $(M C)$}

Simulated data are computed following hypotheses on the modal content (duct modes), which concerns broadband and emergence levels, and energy repartition between the different modes. For this purpose, a state-of-the-art modal 
content used by Airbus is introduced. A broadband level is set for all azimuthal order $m$ and radial order $n$ for which the energy is equidistributed on all radial orders $n$ for each azimuthal order $m$. Moreover, an emergence level corresponding to the engine order is set, as well as two customizable emergences, and one corresponding to the first azimuthal order $m=0$. For all emergences, the energy is carried out by the first radial order $n=1$. Then, a number of azimuthal orders $N_{\text {adj }}$ from each side of each emergences is set, for which the acoustic energy decays as a function of the azimuthal order $m$. In this paper, the mean modal content $\underline{I}_{\alpha}^{+}$, which can be written as $\underline{I}_{m, n}^{+}$, is represented by the quantity $I_{m}$ that corresponds to $\underline{I}_{m, 1}^{+}$(the notation is simplified for facilitating the reading). Quantity $I_{m}$ (that is the mean modal intensity), will also be called "modal content" (MC). The following notations/symbols will be used:

- HW is for "Hardwall", CUT is for "Cutback condition", and $f=946 \mathrm{~Hz}$ is the corresponding frequency.

- $I_{\mathrm{EO}}$ is the modal intensity of the engine order (EO) of azimuthal order $m=m_{\mathrm{EO}}$.

- $I_{\mathrm{FE}^{+}}$is the modal intensity of the positive flexible emergence $\left(\mathrm{FE}^{+}\right)$of azimuthal order $m=m_{\mathrm{FE}^{+}}$.

- $I_{\mathrm{FE}^{-}}$is the modal intensity of the negative flexible emergence $\left(\mathrm{FE}^{-}\right)$of azimuthal order $m=m_{\mathrm{FE}^{-}}$.

- IZero is the modal intensity of the azimuthal order $m=0$ emergence.

- $I_{\mathrm{BB}}$ is the modal intensity of the broadband (BB).

- "Adjacent" is the number $N_{\text {adj }}$ of adjacent modes (see the definition given hereinbefore).

\section{Experimental identification of the modal content}

The modal content (MC) is identified by comparing experimental data with the simulation for the HW case (no liner) and for the cutback condition at the blade passing frequency (BPF) at $f=946 \mathrm{~Hz}$. A trial algorithm has been used for minimizing the difference between simulation and experimental SPL quantities. The nominal MC is modified and yields the so called "modified MC".



Fig. 5 Modified MC resulting from the experimental identification for the cutback condition at the blade passing frequency (BPF) at $f=946 \mathrm{~Hz}$. One graduation equals $5 \mathrm{~dB}$.

Fig. 5 shows the modified MC resulting from the experimental identification process for which it can be seen the broadband level (blue), one positive azimuthal (cyan) and one negative azimuthal emergence (green), one emergence for the first mode (magenta), and one emergence corresponding to the mode carrying the engine order (red). Fig. 6 shows the SPL for the ring acoustic pressures and for the far-field acoustic pressures corresponding (i) to experimental data (blue lines) (ii) to the simulation using the nominal MC (black line), and (iii) to the simulation using the modified MC that results from the experimental identification (red line).

\section{Sensitivity analysis with respect to uncertainties}

This section presents results of the sensitivity analysis with respect to the level of uncertainties given by the values of $\delta_{A}$ and $\delta_{Z}$, in order to assess the impact of aeroacoustic uncertainties and liner uncertainties on the ring and the far-field 



Fig. 6 Ring SPL (top) and Far-field SPL (down): comparison of experiments with simulations. One graduation equals $10 \mathrm{~dB}$.

acoustic pressure. The cutback condition at the blade passing frequency $f=946 \mathrm{~Hz}$ is considered. For the sensitivity to the aeroacoustic uncertainties, the HW case is considered while for the sensitivity to the liner uncertainties, the SW case (lined case) is considered (since there is the liner). The modified MC defined in Fig. 5] is used. For this modified MC, the confidence regions of the random SPL are estimated with the stochastic computational model using Eq. 25] for the ring acoustic pressure and Eq. (26) for the far-field acoustic pressure. In all the figures, the confidence regions that are shown have been estimated for a probability level of 0.95 and using 50 realizations for the stochastic solver (this value gives an acceptable convergence of the confidence regions, convergence that has been checked in increasing this value). The experimental measurements are displayed and consequently, can be compared to the confidence regions that are estimated with the stochastic computational model.

\section{A. Aeroacoustic uncertainties}



(a) Ring SPL



(b) Far-field SPL

Fig. 7 HW case: confidence regions of $\mathrm{SPL}_{\mathrm{ring}, m}$ (a) and $\mathrm{SPL}_{\mathrm{ff}, i}\left(\right.$ b) as function of $\delta_{A}$ compared to experiments. One graduation equals $10 \mathrm{~dB}$ for (a) and $5 \mathrm{~dB}$ for (b).


(a)) and of $\mathrm{SPL}_{\mathrm{ff}, i}$ (figure (b)), estimated with the stochastic computational model, in function of the aeroacoustic 
uncertainty level $\delta_{A} \in\{0.01,0.05,0.75,0.10\}$. These two figures also show the experimental measurements (blue lines) and the corresponding deterministic simulation without uncertainties (black lines). The impact of aeroacoustic uncertainties can be observed in Fig. 77.(a) for the ring SPL and in Fig. 7. (b) for the far-field SPL. It has been found that the sensitivity of SPL $\mathrm{ring}_{\mathrm{m}}$ is a logarithm function of $\delta_{A}$ while the sensitivity of the far-field SPL is a linear function of $\delta_{A}$ (see [8]). Nevertheless, what is more important here is that, in Fig. 77-(b), it can be observed an increase of the acoustic energy due to uncertainties in the $\left[40^{\circ}, 70^{\circ}\right]$ angles range. This will be accounted for during the identification of the uncertainty levels.

\section{B. Liner uncertainties}

For the SW case, similarly to the aeroacoustic uncertainties sensitivity analysis, Fig. 8 shows the sensitivity of the confidence region (CR) of SPL ring, $m$ (figure (a)) and of SPL $\mathrm{ff}_{i}$ (figure (b)), estimated with the stochastic computational model, in function of the liner uncertainty level $\delta_{Z} \in\{0.1,0.3,0.5,0.7\}$, keeping aeroacoustic uncertainties to zero. These two figures also show the experimental measurements (blue lines) and the corresponding deterministic simulation without uncertainties (black lines). As it can be seen, the liner uncertainties have a small impact on the ring SPL,

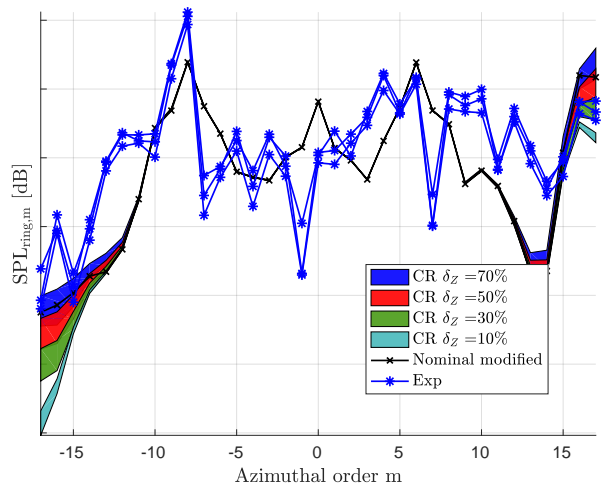

(a) Ring SPL



(b) Far-field SPL One graduation equals $5 \mathrm{~dB}$ for (a) and (b).

(a) and $\mathrm{SPL}_{\mathrm{ff}, i}$ (b) as function of $\delta_{Z}$ compared to experiments.

especially for azimuthal orders in the range $[-12,12]$, whereas outside this range, the SPL is slightly affected. The high cut-off ratio modes are more inclined to be affected by uncertainties since they are poorly energetic. Concerning the far-field SPL, the impact is greater but remains low. The small impact for the ring and far-field SPL can also be explained by the fact that the characteristics of the liner used in this analysis have been chosen close to the characteristics that have been previously optimized by the manufacturer for the flight condition/frequency that is considered here.

\section{Experimental identification of uncertainty levels}

As the liner has to be optimized for several engine regimes (e.g flight conditions), it is important to validate the identification method for at least one engine regime. Moreover, since the optimization of liners is presented only for the cutback condition, the identification of uncertainties is of primary importance using experimental data. The cutback condition at the blade passing frequency $f=946 \mathrm{~Hz}$ is again considered. The confidence regions (CR) of $\mathrm{SPL}_{\mathrm{ring}, m}$ and $\mathrm{SPL}_{\mathrm{ff}, i}$ are estimated with the stochastic computational model for each fixed value of the hyperparameters, for a probability level of 0.95 and using 50 realizations. Taking into account the computational costs, a very simple trial algorithm allowing to control the CPU time has been used. The objective is to frame a maximum number of experimental points within the confidence regions.

\section{A. Experimental identification of the aeroacoustic uncertainty level}

The HW case (no liner) is considered for performing the identification of the aeroacoustic uncertainty level $\delta_{A}$. The modified modal content, which has experimentally been identified in Section VI.C.2 and defined in Fig. 5, is used. The 
optimal aeroacoustic uncertainty level is $\delta_{A}^{\mathrm{opt}}=0.02$.


Fig. 9 HW case: for $\delta_{A}^{\text {opt }}=0.02$, confidence regions of $\mathrm{SPL}_{\mathrm{ring}, m}$ (top) and $\mathrm{SPL}_{\mathrm{ff}, i}$ (down), compared to experiments. One graduation equals $20 \mathrm{~dB}$ (top) and $5 \mathrm{~dB}$ (down).

For the HW case, Fig. 9 shows the experimental measurements (blue lines), the corresponding deterministic simulations without uncertainties (black lines), the confidence region (CR), and their statistical mean values (red lines) of random variables $\mathrm{SPL}_{\mathrm{ring}, m}$ (top figure) and of SPL $\mathrm{ff}_{i}$ (down figure) estimated with the stochastic computational model for the optimal value $\delta_{A}^{\mathrm{opt}}$ of the aeroacoustic uncertainty level. It can be seen that for both ring and far-field microphones, the confidence region frames a low amount of experimental points. Note that for the far-field that is the most important target, there is an angles range between $40^{\circ}$ and $60^{\circ}$ for which aeroacoustic uncertainties have a strong impact. This is a difficulty that induces a conflict with the identification of the modified modal content (MC); there is a set of modes that radiate in this specific angles range and that are very sensitive to aeroacoustic uncertainties.


Fig. 10 SW case: for $\delta_{A}^{\mathrm{opt}}=0.02$ and $\delta_{Z}=0$, confidence regions of $\mathrm{SPL}_{\mathrm{ring}, m}$ (top) and $\mathrm{SPL}_{\mathrm{ff}, i}$ (down), compared to experiments. One graduation equals $20 \mathrm{~dB}$ (top) and $10 \mathrm{~dB}$ (down). 
Before adding the liner uncertainties (see Section VIII.B), in order to analyze the impact of aeroacoustic uncertainty for $\delta_{A}^{\text {opt }}=0.02$ on the configuration with liner (SW case), but without liner uncertainties $\left(\delta_{Z}=0\right.$ ), Fig. 10 shows the experimental measurements (blue lines), the confidence region (CR) of SPL $\mathrm{ring}_{, m}$ (top figure) and of SPL $\mathrm{ff}, i_{\text {(down }}$ figure), their statistical mean values (red lines), and the corresponding deterministic simulations without uncertainties (black lines). It can be observed that on the ring microphone array, for azimuthal orders in the range $[-12,12]$, uncertainties have low impact, as it has already been observed in Section VII.B. Modal content uncertainties will then be needed to frame experimental points in this specific azimuthal order range.

\section{B. Experimental identification of the liner uncertainty level}

For this identification, the SW case is considered for performing the identification of the liner uncertainty level $\delta_{Z}$. The modified modal content, which has experimentally been identified in Section VI.C.2 and defined in Fig. 5 . is used. The aeroacoustic uncertainty level is fixed to the optimal value $\delta_{A}^{\mathrm{opt}}=0.02$ identified in Section VIII.A. As it has been seen during the sensitivity analysis of liner uncertainty level in Section VII.B, liner uncertainties have a relatively small impact on SPL. The optimization of $\delta_{Z}$ is therefore difficult to make and we have preferred fixed a reasonable level of the liner uncertainty level coming from expertise, $\delta_{Z}^{\mathrm{opt}}=0.3$. For the SW case, Fig. 11 shows the


Fig. 11 SW case: for $\delta_{A}^{\text {opt }}=0.02$ and $\delta_{Z}=0.3$, confidence regions of $\mathrm{SPL}_{\mathrm{ring}, m}$ (top) and $\mathrm{SPL}_{\mathrm{ff}, i}$ (down), compared to experiments. One graduation equals $20 \mathrm{~dB}$ (top) and $10 \mathrm{~dB}$ (down).

experimental measurements (blue lines), the corresponding deterministic simulations without uncertainties (black lines), the confidence region (CR) of SPL $\mathrm{Sing}_{m}$ (top figure) and of $\mathrm{SPL}_{\mathrm{ff}, i}$ (down figure), and their statistical mean values (red lines), estimated with the stochastic computational model with $\delta_{A}^{\mathrm{opt}}=0.02$ for the aeroacoustic uncertainty level and with $\delta_{Z}^{\text {opt }}=0.3$ for the liner uncertainty level. It can be seen that, in presence of liners, for both ring and far-field microphones, the confidence region frames a low amount of experimental points. The confidence region estimated using both aeroacoustic and liner uncertainties is not sufficiently large for configuration with liners (that was not the case for the hardwall configuration analyzed in Section VIII.A. The adding of liner uncertainties does not impact the confidence region width at all, which is not surprising regarding what has been observed during the sensitivity analysis (see SectionVII.B. It means that modal content uncertainties have to be introduced.

\section{Experimental identification of the modal content uncertainty level}

The modified modal content, $I_{m}$, that is the mean modal intensity defined in Section VI.C.1, has experimentally been identified in Section VI.C.2 and is defined in Fig. 5 The probabilistic model of modal intensity $I_{\alpha}^{+}$is defined by Eq. (19) for which the mean intensity model $\underline{I}_{\alpha}^{+}$is chosen as the modified modal content $I_{m}$ and for which the hyperparameter is $\sigma_{\alpha}$, which allows the level of uncertainties to be controlled. The objective of the experimental identification of the modal content uncertainty level is to identify $\sigma_{\alpha}(\omega)$ for the SW case in presence of aeroacoustic and 
liner uncertainties whose levels are fixed to the values $\delta_{A}^{\mathrm{opt}}=0.02$ and $\delta_{Z}^{\mathrm{opt}}=0.3$. We recall that we always consider the cutback condition at the blade passing frequency $f=946 \mathrm{~Hz}$. Hyperparameter $\sigma_{\alpha}(\omega)$ is reparameterized introducing the two hyperparameters $\sigma_{\mathrm{EO}}$ and $\sigma_{\mathrm{BB}}$ for the Engine Order and for the Broad Band which become the two parameters that we have to identified. The optimal values $\sigma_{\mathrm{EO}}^{\mathrm{opt}}=\sigma_{\mathrm{BB}}^{\mathrm{opt}}=5 \mathrm{~dB}$ (expressed in $\mathrm{dB}$ ). Fig. 12 displays the 50 realizations of the azimuthal stochastic modal content that has been experimentally identified, depicted, on which the variability on each azimuthal order is shown ( $\pm 5 \mathrm{~dB}$ for broadband modes and $\pm 5 \mathrm{~dB}$ for emergences). For the SW case, Fig. 13



Fig. 12 Realizations of the optimized random $\mathrm{MC}$ obtained for $\sigma_{\mathrm{EO}}^{\mathrm{opt}}=5 \mathrm{~dB}$ and $\sigma_{\mathrm{BB}}^{\mathrm{opt}}=5 \mathbf{d B}$ (one graduation equals $10 \mathrm{~dB})$.


Fig. 13 SW case: for $\delta_{A}^{\text {opt }}=0.02, \delta_{Z}=0.3$, and Fig. 12 confidence regions of SPL $\mathrm{ring}_{\mathrm{r}, m}$ (top) and $\mathrm{SPL}_{\mathrm{ff}, i}$ (down), compared to experiments. One graduation equals $20 \mathrm{~dB}$ (top) and $10 \mathrm{~dB}$ (down).

shows the experimental measurements (blue lines), the corresponding deterministic simulations without uncertainties (black lines), the confidence region (CR) of random variables SPL $\mathrm{Sing}_{m}$ (top figure) and of $\mathrm{SPL}_{\mathrm{ff}, i}$ (down figure), and their statistical mean values (red lines), estimated with the stochastic computational model, for aeroacoustic uncertainty level $\delta_{A}^{\mathrm{opt}}=0.02$, for liner uncertainty level $\delta_{Z}^{\mathrm{opt}}=0.3$, and for random MC defined in Fig. 12 Qualitatively, the experimental identification can be considered as good enough. There is a sufficient number of experimental points 
inside the confidence region for both the far-field acoustic pressures and the ring acoustic pressures. An important point to notice is that the far-field into the $40^{\circ}$ to $60^{\circ}$ angles range is very sensitive to aeroacoustic uncertainties and grows accordingly. This phenomenon is difficult to compensate with the modified modal content (which is logarithmic by essence). Therefore, aeroacoustic uncertainties have to be kept relatively low to avoid the growing of energy in this specific angles range. That is why, for this case, modal content uncertainties are set to a high value, in order to increase the confidence region thickness and frame a maximum number of experimental points.

\section{Robust optimization of liners under uncertainties}

The robust optimization of the liners under uncertainties is performed for the cutback condition at the blade passing frequency $f=946 \mathrm{~Hz}$ and using the stochastic computational model with the aeroacoustic uncertainty level $\delta_{A}^{\mathrm{opt}}=0.02$, the liner uncertainty level $\delta_{Z}^{\mathrm{opt}}=0.3$, and the random MC defined in Fig. 12. The objective is to maximize the acoustic performance relative to the far-field acoustic pressure with respect to the main parameters of the liners while maximizing the robustness. To select these main parameters of the liner, a far-field acoustic pressure sensitivity analysis has been carried out as a function of the candidate parameters. This analysis made it possible to select the two parameters that are described below and are consistent with the state of knowledge in this area. Two optimization cases are considered: the optimization of the forward fan case (FFC) liner and the optimization of the inlet liner. For each one of these two cases, the optimization parameter is denoted by $\mathbf{w}=\left(w_{1}, w_{2}\right)($ there are two design parameters for each case). For the FFC liner, the optimization parameters of the liner are geometrical parameters related to the perforated resistive sheet. Parameter $w_{1}>0$ (denoted as POA) is the percentage of open area while parameter $w_{2}>0$ (denoted as DIA) is the diameter of the facesheet perforations. For the inlet liner, the optimization parameters of the liner are acoustical parameters related to the facesheet (wiremesh). Parameter $w_{1}>0$ (denoted as $R_{20}$ ) is the resistance at the given effective acoustic particle velocity $0.2 \mathrm{~m} / \mathrm{s}$ while parameter $w_{2}>0$ (denoted as NLF) is the non-linearity factor (the definition of the non-linearity factor can be found in Appendix C of [54]).

The design set $C_{\mathbf{w}}$, that is to say, the admissible set of the design parameter $\mathbf{w}$ for the optimization problem is constructed using the following parameterization of $\mathbf{w}$,

$$
w_{k}=\left(1+p_{w_{k}}\right) w_{k}^{\text {nom }} \quad, \quad k=1,2
$$

in which, for $k=1,2, p_{w_{k}}$ is a parameter with values in a given bounded interval of $\mathbb{R}$ and where $w_{k}^{\text {nom }}$ corresponds to a given and fixed nominal value of parameter $w_{k}$. This means that the design set $C_{\mathbf{w}}$ is a rectangular domain.

Since the frequency is fixed to the blade passing frequency, $\omega$ is fixed and thus is removed for simplifying the writing. The acoustic performance of the liners with respect to the HW case will be quantified by the absorption produced by the SW case on the $N_{\text {mics,ff }}$ microphones located in the far field. For the ensemble of all the $N_{\text {mics,ff }}$ microphones located in the far field, the absorption is the positive-valued random variable $\Delta \mathrm{SPL}_{\mathrm{ff}}(\mathbf{w})$ defined by

$$
\Delta \mathrm{SPL}_{\mathrm{ff}}(\mathbf{w})=\frac{1}{N_{\text {mics }, \mathrm{ff}}} \sum_{i=1}^{N_{\text {mics, ff }}}\left(\mathrm{SPL}_{\mathrm{ff}, i}^{\mathrm{HW}}(\mathbf{w})-\mathrm{SPL}_{\mathrm{ff}, i}^{\mathrm{SW}}(\mathbf{w})\right),
$$

in which we have indicated the dependence on the design parameter $\mathbf{w}$ and where $\mathrm{SPL}_{\mathrm{ff}, i}^{\mathrm{HW}}(\mathbf{w})$ and $\mathrm{SPL}_{\mathrm{ff}, i}^{\mathrm{SW}}(\mathbf{w})$ are the far-field sound pressure level SPL $\mathrm{ff}_{, i}$ for microphone $i$ defined by Eq. 26, , for the HW case and the SW case, respectively.

The acoustic performance, $J_{\text {perf }}(\mathbf{w})$, is defined as the following statistical mean value,

$$
J_{\text {perf }}(\mathbf{w})=E\left\{\Delta \operatorname{SPL}_{\mathrm{ff}}(\mathbf{w})\right\}
$$

in which $E$ is the mathematical expectation that is estimated using the realizations. It can be seen that the performance function $J_{\text {perf }}$ is positive, and consequently, the optimization of the acoustic performance of the liners consists in maximizing the positive-valued function $\mathbf{w} \mapsto J_{\text {perf }}(\mathbf{w})$.

The standard deviation $J_{\text {std }}(\mathbf{w})$ of random variable $\Delta \operatorname{SPL}_{\mathrm{ff}}(\mathbf{w})$ is classically defined by

$$
J_{\text {std }}(\mathbf{w})=\left\{E\left\{\left(\Delta \operatorname{SPL}_{\mathrm{ff}}(\mathbf{w})-J_{\text {perf }}(\mathbf{w})\right)^{2}\right\}\right\}^{1 / 2} .
$$


The optimization of the robustness consists in minimizing the positive-valued function $\mathbf{w} \mapsto J_{\text {std }}(\mathbf{w})$.

The global optimization of the liner design consists in finding a compromise between the maximization of the performance function $w \mapsto J_{\text {perf }}(\mathbf{w})$ on $C_{\mathbf{w}}$ and the maximization of the robustness, that is to say, the minimization of function $\mathbf{w} \mapsto J_{\text {std }}(\mathbf{w})$ on $C_{\mathbf{w}}$. Taking into account the CPU time generated by computation, the optimization has been performed by a trial algorithm that starts by using a coarse discretization of $C_{\mathrm{w}}$ for localizing a subset of $\mathcal{C}_{\mathrm{w}}$, followed by a refinement of this subset for improving the construction of the optimal solution.

\section{A. Optimization of the forward fan case (FFC) liner}

We consider the same discretization $\{-0.9,-0.5,-0.3,0,0.3,0.5,0.9\}$ for $\mathbf{p}_{\mathrm{POA}}$ and $\mathbf{p}_{\mathrm{DIA}}$ as the coarse discretization of $C_{\mathrm{w}}$. Fig. 14 displays the standard deviation of random $\mathrm{SPL}_{\mathrm{ff}, i}$ as a function of the microphone $i$ (expressed in angle



Fig. 14 Standard deviation $J_{\mathrm{std}}$ (STD on vertical axis) of $\mathrm{SPL}_{\mathrm{ff}, i}$ as a function of angle $i$ for several values of POA and DIA. One graduation is $0.5 \mathrm{~dB}$

position of the microphone), for several values of the FFC liner parameters POA and DIA. Each graph corresponds to a value of the couple $\left(p_{\text {POA }}, p_{\text {DIA }}\right)$ expressed in percent. For instance, "POA,DIA $=-90,0$ " means $p_{\text {POA }}=-0.9$ and $p_{\text {DIA }}=0$. The dynamic in this graph is $4 \mathrm{~dB}$. This figure, which is related to the robustness of the predictions of the far-field pressure, makes it possible to confirm that the variations of the SPL are indeed related to the variations of POA and DIA and not to numerical artifacts. Also, as observed during the identification of uncertainty levels, in the $40^{\circ}$ to $70^{\circ}$ angles range, the standard deviation admits a maximum, which indicates that uncertainties have a strong impact in this specific region. Moreover, one can observe that the FFC liner design changes, in this specific configuration, have an impact on the acoustic response from $60^{\circ}$ and not much before. This indicates that the FFC liner design is quite robust in the first half of the angles range and more sensitive to design changes in the second half. Finally, curves are grouped by colors (at iso DIA), and not by symbols (at iso POA), which means that the DIA has less impact than the POA. For the coarse discretization of the design set, Fig. 15 . (a) displays the performance function $J_{\text {perf }}$ defined by Eq. (29) that exhibits a dynamic of $4 \mathrm{~dB}$. The star in the region defined by $p_{\text {DIA }} \in[-0.6,-0.3]$ and $p_{\text {POA }} \in[0.5,0.9]$ is an optimum in terms of the acoustic performance of the FFC liner. Fig. 15. (b) shows the standard deviation function $J_{\text {std }}$ defined by Eq. [30, which is related to the robustness and which exhibits a dynamic of $0.6 \mathrm{~dB}$. As it can be seen, there are two regions in which the standard deviation is minimum. One in the region defined by $p_{\mathrm{DIA}} \in[0.6,0.9]$ and $p_{\mathrm{POA}} \in[0,0.3]$, and another in the region defined by $p_{\mathrm{DIA}} \in[-0.9,-0.3]$ and $p_{\mathrm{POA}} \in[0.5,0.9]$. The optimum for the performance and the robustness of the FFC liner is therefore the region defined by $p_{\text {DIA }} \in[-0.55,-0.25]$ and $p_{\mathrm{POA}} \in[0.5,0.8]$.

For the refined discretization of the subset of the design set, Fig. 16 (a) displays the performance function $J_{\text {perf }}$ and Fig. 16-(b) the standard deviation function $J_{\text {std }}$ on the subset identified in Fig. 15. The final optimum of the FFC liner design, corresponding to a compromise between performance and robustness, is $p_{\mathrm{DIA}}^{\text {opt }}=-0.45$ and $p_{\mathrm{POA}}^{\mathrm{opt}}=0.6$. It should be noted that the identified optimum corresponds to a partial optimization that is related to a particular configuration at 




(a) Performance function $J_{\text {perf }}$



(b) Standard deviation function $J_{\text {std }}$

Fig. 15 Coarse discretization: 2D plot of $J_{\text {perf }}(\mathbf{a})$ and $J_{\text {std }}(\mathbf{b})$ in function of $p_{\mathrm{DIA}}(\mathrm{DIA} \pm \mathrm{x} \%)$ and $p_{\mathrm{POA}}(\mathrm{POA} \pm \mathrm{x} \%)$, in percent.



(a) Performance function $J_{\text {perf }}$



(b) Standard deviation function $J_{\text {std }}$

Fig. 16 Refined discretization: 2D plot of $J_{\text {perf }}(\mathbf{a})$ and $J_{\text {std }}$ b) in function of $p_{\text {DIA }}(\mathrm{DIA} \pm \mathrm{x} \%)$ and $p_{\mathrm{POA}}(\mathrm{POA} \pm \mathrm{x} \%)$, in percent.

a single frequency, and consequently, is not an optimum for all the configurations and frequencies.

\section{B. Optimization of the inlet liner}

We consider the same discretization $\{-0.9,-0.5,-0.3,0,0.3,0.5,0.9,1.5\}$ for $\mathbf{p}_{R_{20}}$ and $\mathbf{p}_{\mathrm{NFL}}$ as the coarse discretization of $C_{\mathrm{w}}$. Fig. 17]displays the standard deviation of $\mathrm{SPL}_{\mathrm{ff}, i}$ as a function of the angle $i$ (expressed in angle position of the microphone), for several values of the inlet liner parameters, $R_{20}$ and NLF. Each graph corresponds to a value of the couple $\left(p_{R_{20}}, p_{\mathrm{NLF}}\right)$ expressed in percent. For instance, "R20,NLF $=-90,-90 "$ means $p_{R_{20}}=-0.9$ and $p_{\mathrm{NLF}}=-0.9$. The dynamic in this graph is $2.5 \mathrm{~dB}$. As for the FFC liner, this figure, which is related to the robustness of the predictions of the far-field pressure, makes it possible to confirm that the variations of the SPL are related to the variations of $R_{20}$ and NLF and not to numerical artifacts. Similarly to the FFC liner, the standard deviation admits a maximum in the $40^{\circ}$ to $70^{\circ}$ angles range. This indicates that inlet liner design is quite robust in the first half of the angles range and more sensitive to design changes in the second half. Finally, the curves are grouped by colors (at iso NLF), and not by 




Fig. 17 Standard deviation $J_{\text {std }}$ (STD on vertical axis) of SPL $L_{\mathrm{ff}, i}$ as a function of angle $i$ for several values of $R_{20}$ and NLF. One graduation is $0.5 \mathrm{~dB}$

symbols (at iso $R_{20}$ ), which means that NLF has less impact than the $R_{20}$.



(a) Performance function $J_{\text {perf }}$



(b) Standard deviation function $J_{\text {std }}$

Fig. 18 Coarse discretization: 2D plot of $J_{\text {perf }}\left(\right.$ a) and $J_{\text {std }}(b)$ in function of $p_{R_{20}}\left(R_{20} \pm x \%\right)$ and $p_{\mathrm{NLF}}(\mathrm{NLF} \pm \mathrm{x} \%)$, in percent.

For the coarse discretization of the design set, Fig. 18. (a) displays the performance function $J_{\text {perf }}$ defined by Eq. 291) that exhibits a dynamic of $3 \mathrm{~dB}$. The optimum for the acoustic performance of the inlet liner belongs to the subset defined by $p_{R_{20}} \in[-0.2,0.2]$ and $p_{\mathrm{NLF}} \in[1.1,1.5]$. Fig. 18 . (b) shows the standard deviation function $J_{\text {std }}$ defined by Eq. 30, which is related to the robustness and which exhibits a dynamic of $0.24 \mathrm{~dB}$. For the refined discretization of the subset of the design set, Fig. 19. (a) displays the performance function $J_{\text {perf }}$ with a dynamic of $0.32 \mathrm{~dB}$ and Fig. 19. (b) the standard deviation function $J_{\text {std }}$ with a dynamic of $0.24 \mathrm{~dB}$, estimated on the subset identified in Fig. 18 The final optimum of the FFC liner design, corresponding to a compromise between performance and robustness, is $p_{\text {DIA }}^{\text {opt }}=-0.45$ and $p_{\mathrm{POA}}^{\mathrm{opt}}=0.6$. It should be noted that the found optimum corresponds to a partial optimization that is related to a particular configuration at a single frequency, and consequently, is not an optimum for all the configurations and frequencies. It can be seen that the region $p_{R_{20}} \in[-0.02,0.02]$ and $p_{\mathrm{NLF}} \in[1.12,1.3]$ corresponds to a good 




(a) Performance function $J_{\text {perf }}$



(b) Standard deviation function $J_{\text {std }}$

Fig. 19 Refined discretization: 2D plot of $J_{\text {perf }}\left(\right.$ a) and $J_{\text {std }}\left(\right.$ b) in function of $p_{R_{20}}\left(R_{20} \pm x \%\right)$ and $p_{\mathrm{NLF}}(\mathrm{NLF} \pm \mathrm{x} \%)$, in percent

compromise between performance and robustness, as the performance in this region is constant (and optimum), and the robustness is maximum in the subregion $p_{R_{20}} \in[-0.02,0.02]$ and $p_{\mathrm{NLF}} \in[1.15,1.20]$, which is included in the acoustic performance optimum region. The optimum for this inlet liner is then $p_{R_{20}}^{\mathrm{opt}}=0$ and $p_{\mathrm{NLF}}^{\mathrm{opt}}=1.25$. Parameter $R_{20}$ has the greatest influence on the performance of the inlet liner and its optimal value corresponds to the nominal value. Parameter NLF has less influence on the performance of the inlet liner.

\section{Discussion}

For only one flight condition (cutback) and one frequency (1 BPF), the FFC liner and the inlet liner have been optimized in presence of uncertainties for which their levels have been experimentally identified in Section VIII. One objective was to compare the nominal liners designed by Airbus for which a full state-of-the-art optimization analysis was performed, with the present analysis that is simply related to a single flight condition and for a single frequency. For obvious reasons, it is not possible to compare both optima in the same conditions. Nevertheless, this comparison gives an idea of what can be obtained with the stochastic computational aeroacoustic model, and more importantly, allows for verifying that the optimum found with this robust approach is consistent with the nominal design. Concerning the optimization of the FFC liner, the POA design parameter of the liner is clearly more impacting on the acoustic performance than the DIA design parameter. This leads us to a one sided optimization study in terms of the acoustic performance. For the robustness, the DIA parameter has a slightly better impact, but enough to allow for finding an optimum. The inlet liner has a higher acoustic surface and also has a different facesheet definition (wiremesh instead of a perforated plate for the FFC). Concerning its optimization, even if the $R_{20}$ design parameter has a significantly better impact on the acoustic absorption, the NLF impacts allows also a clear optimal region to be identified for the acoustic performance, and even better for the design robustness. For optimization of both the FFC liner and the inlet liner, a compromise had to be made between acoustic performance in terms of absorption and robustness of the design. Even if we managed to find both optima in similar regions each time, it will not be that easy in further studies when several frequencies, several flight conditions, and more design parameters will have to be taken into account. Nevertheless, the blade passing frequency being a crucial design frequency for the liner design, the presented results are quite promising. One important point is that the stochastic computational aeroacoustic model seems to correctly work, and gives for the specific studied configuration, results that are not far away from the nominal value used by Airbus. Nevertheless, as it has been explained, the results could be better if the modal content was improved, which would allow a more precise identification of aeroacoustic and liner uncertainties to be carried out. 


\section{Conclusion}

This work is devoted to the implementation of a robust design process allowing for associating with the acoustic performance of a given liner design, its propensity to keep its optimum performance stable to an external variability. A first goal has been to track and quantify the main sources of uncertainties that lie within the numerical tool used for the dimensioning of liner following its acoustic performance. The variability has been accounted for under the form of uncertainties within the computational aeroacoustic model, which are induced by modeling errors.

In order to account for such a variability, a novel quantification of uncertainties for such a complex aeroacoustic problem has been presented. Two main operators have been constructed related to the liner impedance and the aeroacoustic operator responsible for the acoustic effects induced by the flow. The nonparametric probabilistic approach of uncertainties induced by modeling errors has been developed for each operator, based on their intrinsic algebraic properties, related to the energy conservation/dissipation properties. This approach consists in introducing random fluctuations to the operators. The uncertainties on the acoustic excitation induced by the fan, that is represented by the modal content corresponding to the modal expansion on duct modes, has been performed using the classical parametric approach of uncertainties. The levels of uncertainties for the aeroacoustic operator, the liner operator, and the modal content are controlled by hyperparameters that allow for imposing a percentage of uncertainties and have been identified solving a statistical inverse method that consists in comparing the stochastic acoustic response of the computational model with experimental measurements.

Once uncertainty levels and modal content have been fixed, an optimization of two liner surfaces has been carried out with the stochastic computational aeroacoustic model involving uncertainty models. For solving the optimization problem that is CPU time consuming, a coarse design set of the liner design parameters has been drawn by taking proportions of the nominal (optimum value found by Airbus) from each side of the design parameters, then a refined design set has been taken around the first updated optimum resulting from the coarse design set investigation. The state-of-the-art optimization process used by Airbus consists in giving an information about the acoustic performance of a given design (set of design parameters). What this work provides, is an information, in addition to the performance information, about the propensity of a given liner design to keep its acoustic performance stable to small changes that impact its design parameters. Namely, an information about the robustness of a given design. This information, allied to the performance, allows for choosing a design in early phases of an aircraft development, for which the acoustic performance would not be impacted by small changes induced by the increasing maturity of the aircraft. Such a maturity increasing would then be anticipated in early phases and would save some re-optimization activities that are time consuming. Moreover, this work allowed for an analysis the uncertainty that lies within the computational aeroacoustic model, that has never been done. As the inverse identification and optimization have only been carried out for one flight condition and a single frequency, and as the Airbus state-of-the-art optimization process is much more richer in terms of flight conditions and frequencies, the optimization results of the liners cannot directly be compared.

Finally, although the proposed approach be mainly dedicated to the preconception phases of the nacelle design, the developed probabilistic method is interesting and usable for the final phase of the design, in order to take into account all the modeling uncertainties.

\section{References}

[1] Ghanem, R., Higdon, D., and Owhadi, H., Handbook of Uncertainty Quantification, Vol. 1 to 3, Springer, Cham, Switzerland, 2017. doi:https://doi.org/10.1007/978-3-319-12385-1.

[2] Jones, M., Parrott, T., and Watson, W., "Uncertainty and sensitivity analyses of a two-parameter impedance prediction model," 14th AIAA/CEAS Aeroacoustics Conference (29th AIAA Aeroacoustics Conference), 2008, p. 2928.

[3] Brown, M., Jones, M., and Watson, W., "Uncertainty analysis of the grazing flow impedance tube," 18th AIAA/CEAS Aeroacoustics Conference (33rd AIAA Aeroacoustics Conference), 2012, p. 2296.

[4] Nark, D. M., Jones, M. G., and Piot, E., "Assessment of axial wave number and mean flow uncertainty on acoustic liner impedance eduction,” 2018 AIAA/CEAS Aeroacoustics Conference, 2018, p. 3444.

[5] Zhou, L., and Bodén, H., "A systematic uncertainty analysis for liner impedance eduction technology," Journal of sound and vibration, Vol. 356, 2015, pp. 86-99. doi:https://doi.org/10.1016/j.jsv.2015.07.001. 
[6] Robinson, J., and Watson, W., "Performance of a checkerboard liner with uncertain impedances," 11th AIAA/CEAS Aeroacoustics Conference, 2005, p. 2850.

[7] Soize, C., Uncertainty Quantification. An Accelerated Course with Advanced Applications in Computational Engineering, Vol. Interdisciplinary Applied Mathematics, Springer, 2017.

[8] Dangla, V., Soize, C., Cunha, G., Mosson, A., Kassem, M., and Van Den Nieuwenhof, B., "Stochastic computational model of 3D acoustic noise predictions for nacelle liners," AIAA AVIATION 2020 FORUM, 2020, p. 2545.

[9] Free Field Technologies, “Actran 12 User’s Guide,”, 2011.

[10] Lighthill, M. J., “On sound generated aerodynamically. I. General theory," Proceedings of the Royal Society of London A: Mathematical, Physical and Engineering Sciences, Vol. 211, The Royal Society, 1952, pp. 564-587.

[11] Lighthill, M. J., Waves in fluids, Cambridge university press, 2001.

[12] Landau, L. D., and Lifshitz, E., Fluid Mechanics: Vol 6, Elsevier, 2013.

[13] Mohring, W., "A well posed acoustic analogy based on a moving acoustic medium,” arXiv preprint arXiv:1009.3766, 2010.

[14] Bruneau, M., and Scelo, T., Fundamentals of Acoustics, ISTE, Wiley, 2010. URL https://books.google.fr/books?id= 8W4TtWhP_nEC

[15] Pierce, A. D., and Beyer, R. T., "Acoustics: An Introduction to Its Physical Principles and Applications. 1989 Edition,”, 1990.

[16] Jones, M., Watson, W., and Parrott, T., "Benchmark data for evaluation of aeroacoustic propagation codes with grazing flow," 11th AIAA/CEAS Aeroacoustics Conference, 2005, p. 2853.

[17] Tam, C. K., “Computational aeroacoustics-Issues and methods,” AIAA journal, Vol. 33, No. 10, 1995, pp. 1788-1796. doi:https://doi.org/10.2514/3.12728.

[18] Tam, C. K., and Dong, Z., "Radiation and outflow boundary conditions for direct computation of acoustic and flow disturbances in a nonuniform mean flow," Journal of computational acoustics, Vol. 4, No. 02, 1996, pp. 175-201. doi: https://doi.org/10.1142/S0218396X96000040.

[19] Tam, C. K., "Computational aeroacoustics: an overview of computational challenges and applications," International Journal of Computational Fluid Dynamics, Vol. 18, No. 6, 2004, pp. 547-567. doi:https://doi.org/10.1080/10618560410001673551.

[20] Tam, C. K., "Advances in numerical boundary conditions for computational aeroacoustics," Journal of Computational Acoustics, Vol. 6, No. 04, 1998, pp. 377-402. doi:https://doi.org/10.1142/S0218396X98000259.

[21] Zhang, Y., Chen, H., Wang, K., and Wang, M., "Aeroacoustic prediction of a multi-element airfoil using wall-modeled large-eddy simulation,” AIAA Journal, 2017, pp. 4219-4233. doi:https://doi.org/10.2514/1.J055853.

[22] Freund, J. B., "Proposed inflow/outflow boundary condition for direct computation of aerodynamic sound," AIAA journal, Vol. 35, No. 4, 1997, pp. 740-742. doi:https://doi.org/10.2514/2.167.

[23] Fahy, F. J., and Gardonio, P., Sound and structural vibration: radiation, transmission and response, Academic press, 2007.

[24] Hirsch, C., Numerical Computation of Internal and External Flows, Fundamentals of Numerical Discretization, Numerical Computation of Internal and External Flows, Wiley, 1991. URL https://books.google.fr/books?id=pswYAQAAIAAJ.

[25] Wagner, C., Hüttl, T., and Sagaut, P., Large-eddy simulation for acoustics, Vol. 20, Cambridge University Press, 2007.

[26] Manoha, E., Troff, B., and Sagaut, P., “Trailing-edge noise prediction using large-eddy simulation and acoustic analogy,” AIAA journal, Vol. 38, No. 4, 2000, pp. 575-583. doi:https://doi.org/10.2514/2.1015.

[27] Bailly, C., and Juvé, D., "Aeroacoustic simulations and stochastic approach using linearized Euler's equations," The Journal of the Acoustical Society of America, Vol. 105, No. 2, 1999, pp. 1066-1066. doi:https://doi.org/10.1121/1.425067.

[28] Bailly, C., and Juve, D., "Numerical solution of acoustic propagation problems using linearized Euler equations," AIAA journal, Vol. 38, No. 1, 2000, pp. 22-29. doi:https://doi.org/10.2514/2.949.

[29] Astley, R., Sugimoto, R., and Mustafi, P., "Computational aero-acoustics for fan duct propagation and radiation. Current status and application to turbofan liner optimisation,” Journal of Sound and Vibration, Vol. 330, No. 16, 2011, pp. 3832-3845. doi:https://doi.org/10.1016/j.jsv.2011.03.022. 
[30] Hirschberg, A., and Rienstra, S. W., "An introduction to aeroacoustics," Eindhoven university of technology, 2004.

[31] Lidoine, S., "Approches théoriques du problème du rayonnement acoustique par une entrée d'air de turboréacteur: Comparaisons entre différentes méthodes analytiques et numériques," Ph.D. thesis, Ecully, Ecole centrale de Lyon, 2002.

[32] Guess, A., "Calculation of perforated plate liner parameters from specified acoustic resistance and reactance," Journal of Sound and Vibration, Vol. 40, No. 1, 1975, pp. 119-137. doi:https://doi.org/10.1016/S0022-460X(75)80234-3.

[33] Parrott, T. L., and Jones, M. G., "Parallel-element liner impedances for improved absorption of broadband sound in ducts," Noise Control Engineering Journal, Vol. 43, No. 6, 1995, pp. 183-195. doi:https://doi.org/10.3397/1.2828379.

[34] Jones, M., Tracy, M., Watson, W., and Parrott, T., "Effects of liner geometry on acoustic impedance," 8th AIAA/CEAS Aeroacoustics Conference \& Exhibit, 2002, p. 2446.

[35] Primus, J., Piot, E., and Simon, F., "An adjoint-based method for liner impedance eduction: Validation and numerical investigation," Journal of Sound and Vibration, Vol. 332, No. 1, 2013, pp. 58-75. doi:https://doi.org/10.1016/j.jsv.2012.07.051.

[36] Tam, C. K., Pastouchenko, N., Jones, M. G., and Watson, W. R., "Experimental validation of numerical simulation for an acoustic liner in grazing flow," 19th AIAA/CEAS Aeroacoustics Conference, 2013, p. 2222.

[37] Zhao, D., Ang, L., and Ji, C., "Numerical and experimental investigation of the acoustic damping effect of single-layer perforated liners with joint bias-grazing flow," Journal of Sound and Vibration, Vol. 342, 2015, pp. 152-167. doi:https: //doi.org/10.1016/j.jsv.2014.12.035.

[38] Zhao, D., Ji, C., Li, J., and Ang, L., "Experimental comparison of noise dissipation effects of single-and double-layer acoustic liners," Applied Acoustics, Vol. 141, 2018, pp. 281-292. doi:https://doi.org/10.1016/j.apacoust.2018.07.024.

[39] Zhao, D., Sun, Y., Ni, S., Ji, C., and Sun, D., "Experimental and theoretical studies of aeroacoustics damping performance of a biasflow perforated orifice," Applied Acoustics, Vol. 145, 2019, pp. 328-338. doi:https://doi.org/10.1016/j.apacoust.2018.10.025.

[40] W. Tam, C. K., and Kurbatskii, K. A., "Microfluid dynamics and acoustics of resonant liners,” AIAA journal, Vol. 38, No. 8, 2000, pp. 1331-1339. doi:https://doi.org/10.2514/2.1132.

[41] Roche, J.-M., Leylekian, L., Delattre, G., and Vuillot, F., "Aircraft fan noise absorption: DNS of the acoustic dissipation of resonant liners," 15th AIAA/CEAS Aeroacoustics Conference (30th AIAA Aeroacoustics Conference), 2009, p. 3146.

[42] Mendez, S., and Eldredge, J. D., "Acoustic modeling of perforated plates with bias flow for large-eddy simulations," Journal of Computational Physics, Vol. 228, No. 13, 2009, pp. 4757-4772. doi:https://doi.org/10.1016/j.jcp.2009.03.026.

[43] Marx, D., and Aurégan, Y., "Effect of turbulent eddy viscosity on the unstable surface mode above an acoustic liner," Journal of Sound and Vibration, Vol. 332, No. 15, 2013, pp. 3803-3820. doi:https://doi.org/10.1016/j.jsv.2013.02.005.

[44] Zhang, Q., and Bodony, D. J., "Numerical investigation of a honeycomb liner grazed by laminar and turbulent boundary layers," Journal of Fluid Mechanics, Vol. 792, 2016, pp. 936-980. doi:https://doi.org/10.1017/jfm.2016.79.

[45] Duchaine, F., "Sensitivity Analysis of Heat Transfer in a Honeycomb Acoustic Liner to Inlet Conditions With Large Eddy Simulation," ASME Turbo Expo 2017: Turbomachinery Technical Conference and Exposition, American Society of Mechanical Engineers, 2017, pp. V05BT22A002-V05BT22A002.

[46] Ramdani, S., Yamasaki, N., Inokuchi, Y., and Ishii, T., "Large Eddy Simulation of Conventional and Bias Flow Acoustic Liners," ASME Turbo Expo 2017: Turbomachinery Technical Conference and Exposition, American Society of Mechanical Engineers, 2017, pp. V02CT43A007-V02CT43A007.

[47] Eldredge, J., Bodony, D., and Shoeybi, M., "Numerical investigation of the acoustic behavior of a multi-perforated liner," 13th AIAA/CEAS Aeroacoustics Conference (28th AIAA Aeroacoustics Conference), 2007, p. 3683.

[48] Pascal, L., Piot, E., and Casalis, G., "A new implementation of the extended Helmholtz resonator acoustic liner impedance model in time domain CAA," Journal of Computational Acoustics, Vol. 24, No. 01, 2016, p. 1550015. doi:https://doi.org/10. 1142/S0218396X15500150.

[49] Zhang, Q., and Bodony, D. J., "Numerical simulation of two-dimensional acoustic liners with high-speed grazing flow," AIAA journal, Vol. 49, No. 2, 2011, pp. 365-382. doi:https://doi.org/10.2514/1.J050597.

[50] Rice, E. J., "Theoretical study of the acoustic impedance of orifices in the presence of a steady grazing flow," The Journal of the Acoustical Society of America, Vol. 59, No. S1, 1976, pp. S32-S32. doi:https://doi.org/10.1121/1.2002637. 
[51] Bauer, A. B., "Impedance theory and measurements on porous acoustic liners," Journal of Aircraft, Vol. 14, No. 8, 1977, pp. 720-728. doi:https://doi.org/10.2514/3.58844.

[52] Maa, D.-Y., "Potential of microperforated panel absorber," the Journal of the Acoustical Society of America, Vol. 104, No. 5, 1998, pp. 2861-2866. doi:https://doi.org/10.1121/1.423870.

[53] Jones, M., and Watson, W., "On the use of experimental methods to improve confidence in educed impedance," 17th AIAA/CEAS Aeroacoustics Conference (32nd AIAA Aeroacoustics Conference), 2011, p. 2865. doi:https://doi.org/10.2514/6.2011-2865.

[54] Dangla, V., "Robust design of acoustic treatment for nacelle noise reduction using computational aeroacoustics and uncertainty quantification,” Ph.D. thesis, Université Gustave Eiffel, 2020. URL https://tel . archives-ouvertes . fr/tel-03115688/ document

[55] Lafronza, L., McAlpine, A., Keane, A., and Astley, J., "Response surface method optimization of uniform and axially segmented duct acoustics liners," Journal of Aircraft, Vol. 43, No. 4, 2006, pp. 1089-1102. doi:https://doi.org/10.2514/1.17727.

[56] Di Francescantonio, P., Casalino, D., and De Mercato, L., "Aeroacoustic design of aero-engine intake liners," 11th AIAA/CEAS Aeroacoustics Conference, 2005, p. 2942.

[57] Van Den Nieuwenhof, B., Detandt, Y., Lielens, G., Rosseel, E., Soize, C., Dangla, V., Kassem, M., and Mosson, A., "Optimal Design of the Acoustic Treatments Damping the Noise Radiated by a Turbo-Fan Engine," 23rd AIAA/CEAS Aeroacoustics Conference, 2017, p. 4035.

[58] Özkaya, E., Gauger, N. R., Hay, J. A., and Thiele, F., "Efficient design optimization of acoustic liners for engine noise reduction," AIAA Journal, Vol. 58, No. 3, 2020, pp. 1140-1156. doi:https://doi.org/10.2514/1.J057776.

[59] Myers, M., "On the acoustic boundary condition in the presence of flow," Journal of Sound and Vibration, Vol. 71, No. 3, 1980, pp. 429-434. doi:https://doi.org/10.1016/0022-460X(80)90424-1.

[60] Brambley, E. J., "Well-posed boundary condition for acoustic liners in straight ducts with flow," AIAA journal, Vol. 49, No. 6, 2011, pp. 1272-1282. doi:https://doi.org/10.2514/1.J050723.

[61] Soize, C., "A nonparametric model of random uncertainties for reduced matrix models in structural dynamics," Probabilistic engineering mechanics, Vol. 15, No. 3, 2000, pp. 277-294. doi:https://doi.org/10.1016/S0266-8920(99)00028-4.

[62] Van Antwerpen, B., Detandt, Y., Copiello, D., Rosseel, E., and Gaudry, E., "Performance improvements and new solution strategies of Actran/TM for nacelle simulations," 20th AIAA/CEAS Aeroacoustics Conference, 2014, p. 2315.

[63] van Den Nieuwenhof, B., Detandt, Y., Lielens, G., Rosseel, E., Soize, C., Dangla, V., Kassem, M., and Mosson, A., "Optimal Design of the Acoustic Treatments Damping the Noise Radiated by a Turbo-Fan Engine," 23rd AIAA/CEAS Aeroacoustics Conference, 2017, p. 4035.

[64] Ohayon, R., and Soize, C., Advanced computational vibroacoustics: reduced-order models and uncertainty quantification, Cambridge University Press, 2014.

[65] Rubinstein, R. Y., and Kroese, D. P., Simulation and the Monte Carlo method, Vol. 10, John Wiley \& Sons, 2016.

[66] Soize, C., "Random matrix theory and non-parametric model of random uncertainties in vibration analysis," Journal of sound and vibration, Vol. 263, No. 4, 2003, pp. 893-916. doi:https://doi.org/10.1016/S0022-460X(02)01170-7.

[67] Soize, C., "Random matrix theory for modeling uncertainties in computational mechanics," Computer methods in applied mechanics and engineering, Vol. 194, No. 12-16, 2005, pp. 1333-1366. doi:https://doi.org/10.1016/j.cma.2004.06.038.

[68] Soize, C., "A comprehensive overview of a non-parametric probabilistic approach of model uncertainties for predictive models in structural dynamics," Journal of sound and vibration, Vol. 288, No. 3, 2005, pp. 623-652. doi:https://doi.org/10.1016/j.jsv. 2005.07.009.

[69] Soize, C., "Random matrix models and nonparametric method for uncertainty quantification," Handbook of Uncertainty Quantification, Vol. 1, edited by R. Ghanem, D. Higdon, and H. Owhadi, Springer, Cham, Switzerland, 2017, pp. $219-287$. doi:https://doi.org/10.1007/978-3-319-11259-6_5-1.

[70] Soize, C., "Random Matrices in Structural Acoustics," New Directions in Linear Acoustics and Vibration: Quantum Chaos, Random Matrix Theory and Complexity, 2010, p. 206. 
[71] Ohayon, R., and Soize, C., "Computational vibroacoustics in low-and medium-frequency bands: damping, ROM, and UQ modeling," Applied Sciences, Vol. 7, No. 6, 2017, p. 586. doi:https://doi.org/10.3390/app7060586.

[72] Arnst, M., and Soize, C., "Identification and sampling of Bayesian posteriors of high-dimensional symmetric positive-definite matrices for data-driven updating of computational models," Computer Methods in Applied Mechanics and Engineering, Vol. 352, 2019, pp. 300-323. doi:https://doi.org/10.1016/j.cma.2019.04.025.

[73] Akkaoui, Q., Capiez-Lernout, E., Soize, C., and Ohayon, R., "Uncertainty quantification for dynamics of geometrically nonlinear structures coupled with internal acoustic fluids in presence of sloshing and capillarity," Journal of Fluids and Structures, Vol. 94, 2020, p. 102966. doi:https://doi.org/10.1016/j.jfluidstructs.2020.102966.

[74] Cottereau, R., Clouteau, D., and Soize, C., "Construction of a probabilistic model for impedance matrices," Computer Methods in Applied Mechanics and Engineering, Vol. 196, No. 17-20, 2007, pp. 2252-2268. doi:https://doi.org/10.1016/j.cma.2006.12.001.

[75] Soize, C., and Poloskov, I. E., "Time-domain formulation in computational dynamics for linear viscoelastic media with model uncertainties and stochastic excitation," Computers \& Mathematics with Applications, Vol. 64, No. 11, 2012 , pp. 3594-3612. doi:https://doi.org/10.1016/j.camwa.2012.09.010.

[76] Capillon, R., Desceliers, C., and Soize, C., "Uncertainty quantification in computational linear structural dynamics for viscoelastic composite structures," Computer Methods in Applied Mechanics and Engineering, Vol. 305, 2016 , pp. 154-172. doi:https://doi.org/10.1016/j.cma.2016.03.012.

[77] Dickson, N., "ICAO noise standards," ICAO Symposium on Aviation and Climate Change, 2013. 


\title{
Supplementary material of the paper:
}

\section{Robust 3D acoustic performance probabilistic model}

\author{
for nacelle liners
}

\section{Journal: AIAA Journal}

\author{
Vincent Dangla * \\ Airbus Operations S.A.S, 31300 Toulouse, France \\ Gustave Eiffel University, 77454 Marne-la-Vallée, France \\ Christian Soize ${ }^{\dagger}$ \\ Gustave Eiffel University, 77454 Marne-la-Vallée, France \\ Guilherme Cunha *, Aurélien Mosson ${ }^{\S}$, Morad Kassem ${ }^{\text {II }}$ \\ Airbus Operations S.A.S, 31300 Toulouse, France \\ Benoit Van Den Nieuwenhof" \\ Free Field Technologies, 1435 Mont-Saint-Guibert, Belgium
}

\section{Introduction}

This Supplementary material corresponds to the extended version of Section II (Construction of the nominal computational model), Section III (Computational reduced-order model), and Section IV (Probabilistic model of uncertainty quantification) of the paper entitled "Robust 3D acoustic performance model for nacelle liners" published in AIAA Journal. In particular, the extended version of these Sections II, III, and IV gives complete developments of

(1) the boundary value problem associated with the inner, outer, and duct domains of the intake acoustic radiation problem (see Section II-D),

(2) the algebraic properties of the complex matrix $[\mathbb{Z}(\omega)]$ related to the liner impedance (see Section III-C) and the algebraic properties of the complex matrix $[\mathbb{A}(\omega)]$ related to the aeroacoustic impedance (see Section III-D),

(3) the construction of the probabilistic model of the reduced liner impedance matrix (see Section IV-B) and the construction of the probabilistic model of the reduced aeroacoustic matrix (see Section IV-C).

All of the developments given in the above mentioned sections have been removed from the article published in the AIAA Journal due to the length limits. However, instead of giving only the sections quoted above, we preferred to give the entire extended version of these sections for ease of reading.

\footnotetext{
*PhD Student, Gustave Eiffel University, MSME UMR 8208 CNRS, 5 bd Descartes, F-77454 Marne-la-Vallée, France; vincent.dangla@ airbus.com.

†Professor, Gustave Eiffel University, MSME UMR 8208 CNRS, 5 bd Descartes, F-77454 Marne-la-Vallée, France; christian.soize@ univ-eiffel.fr (Corresponding Author).

${ }^{\ddagger}$ Research Engineer, Airbus Operations S.A.S, 316 Route de Bayonne, F-31300 Toulouse, France, guilherme.coelho-cunha@ airbus.com.



IIResearch Engineer, Airbus Operations S.A.S, 316 Route de Bayonne, F-31300 Toulouse, France, morad.kassem@airbus.com.

"Senior Acoustic Consultant, Free Field Technologies, 9 rue Emile Francqui, 1435 Mont-Saint-Guibert, Belgique, Benoit.VanDenNieuwenhof@ fft.be.
} 


\section{Nomenclature}






$\begin{array}{ll}\psi_{\mathrm{i}} & =\text { vector of the finite element discretization of field } \psi_{\mathrm{i}} \\ \psi_{\ell} & =\text { vector of all the dofs of } \psi_{\mathrm{i}} \text { related to the liner } \\ \psi_{\mathrm{o}} & =\text { vector of the finite/infinite discretization of field } \psi_{\mathrm{o}} \\ \omega & =\text { angular frequency in rad/s } \\ \Omega_{\mathrm{i}}, \Omega_{\mathrm{o}}, \Omega_{\mathrm{duct}} & =\text { inner, outer, and duct domains of the intake acoustic problem }\end{array}$

\section{Conventions for mathematical notations}

Lower-case letter such as $q$ is a deterministic real variable.

Boldface lower-case letter such as $\mathbf{q}$ is a deterministic vector.

Upper-case letter such as $Q$ (with exception for letters $N$ and $M$ ) is a real-valued random variable.

Boldface upper-case letters such as $\mathbf{Q}$ is a vector-valued random variable.

Upper-case letter between brackets such as $[A]$ is a deterministic matrix.

Boldface upper-case letter between brackets such as $[\mathbf{A}]$ is a matrix-valued random variable.

Note that square brackets are introduced for matrices in order to distinguish a vector from a matrix, a random vector from a random matrix.

Let $[U]$ be any real or complex matrix, then, $[U]^{T}$ is its transpose and $[U]^{*}=\overline{[U]}^{T}$ is its adjoint (transpose and complex conjugate). If $[U]$ is any complex square matrix, then, $\operatorname{tr}[U]$ is its trace, $\|U\|_{F}$ is its Frobenius norm such that $\|U\|_{F}^{2}=\operatorname{tr}\left\{[U]^{*}[U]\right\}$, if $[U]^{T}=[U]$, then $[U]$ is symmetric, and if $[U]^{*}=[U]$, then $[U]$ is Hermitian.

\section{Construction of the nominal (mean) computational model}

\section{A. Framework of the presented methodology}

The theory of acoustic propagation in a fluid flow has been written by authors such as Lighthill [1, 2] who gave birth to the aeroacoustics field of research, Rienstra [3] who developed the theory of acoustic propagation in ducts, and many others, which contributed to the development of fluid dynamic [4, 5] or acoustics [6, 7]. These prediction tools have been created and constantly improved upon since the last century with the work of authors such as Jones [8] on the aeroacoustic propagation, Tam [9-12] on computational aeroacoustic matters such as the acoustic boundary conditions, and others [13-16]. For computational prediction models, the flow inside and outside the nacelle is calculated using CFD (computational fluid dynamics) [17, 18] and allows for identifying the thermodynamical state of the fluid in which the acoustic perturbation propagates. Some works have been devoted to acoustic noise sources generated by the flow itself such as Bailly and Juvé [19, 20] in which a stochastic noise generation process is solved using linearized Euler equation. In the present work, acoustic source terms are neglected. The fan acoustic excitation is represented using a duct modal expansion.

In the framework of nacelle intake liners performance prediction, the following axes can be considered.

1) Noise generation, propagation, and transmission in a moving fluid. In order to understand the mechanisms responsible for the noise production, some studies have been carried out such as [3, 14, 21, 22] on fan noise propagation in duct, and [15] on the transmission/radiation of sound and vibration. This axis is of major importance since knowing the phenomena and mechanisms responsible for acoustic noise in the nacelle allows for developing solutions that are more efficient in mitigating it. In the framework of this paper, the noise generation mainly concerns the study of the interaction between the moving fluid and moving mechanical parts such as the fan or the engine internal systems. The propagation of the noise in a ducted environment for which there is an interaction between the flow and the wall (under the form of a boundary layer) is of major importance when studying lined environments. Finally, the transmission/radiation phenomenon can be studied separately and are not performed in the framework of this paper.

2) Acoustic treatments as mechanical systems. A second axis is related to the mechanical system that constitutes the liner itself. Such a mechanical system is an assembly of several components for which the dissipation phenomena are to be well-modeled so it can be designed to be optimal at specific frequencies (tunable to specific frequencies). The main quantity used for characterizing a liner is its acoustic impedance. The theory of impedance calculation has been introduced by Guess [23] for the mathematical formulation of perforations, or Parrott, Jones, and Watson [24-26] for the assembly of several parts and the effects of liner geometry on its acoustic impedance. In presence of a moving fluid, the dissipation phenomena are more complex and have been studied by several authors, especially at NASA and 
ONERA, using experiments ([27-32]), and/or simulations, such as CFD [33-39] or computational aeroacoustic codes (CAA) [13, 40,42].

3) Acoustic absorption using liners, and liner performance. A final axis concerns the absorption of acoustic perturbation in lined ducts in presence of a moving fluid. This axis is devoted to the optimization of such liners in their operating environment in order to maximize their ability to absorb the noise produced by the fan. Studies have been carried out on optimization methods [43-46] in order to improve their effectiveness in finding the combination of design parameters that is the most adequate to tackle a specific noise frequency range. These improvements concern the computational tools used, the design parameters that are the most impacting, and the choice of representation for the fan acoustic excitation. In [44], the interest of an axially-segmented liner instead of a uniform one is demonstrated, using a multi-modal representation of the fan noise, as it is done in the present work.

\section{B. Summarizing the methodology for constructing the nominal computational model}

First, we will present the boundary value problem associated with 3D inner, outer, and duct domains of the intake acoustic radiation problem. The linear convected Helmhlotz equation [1, 4, 6, 16] is used in the inner domain (near-field) and in the outer domain (far-field), involving the velocity and mass density fields of the flow computed using CFD. The boundary condition on the liner is the Myers condition [22, 47, 48] involving the local admittance of the liner model [23] depending on the spatial point and on the frequency. At infinity, the outward Sommerfeld radiation condition is written. For the fan excitation (see for instance [3] and [7]), the acoustic velocity field is represented on the family of acoustic duct modes and allows for ensuring the coupling between the semi-infinite duct and the inner domain on the fan boundary, and for representing the acoustic excitation produced by the fan. It should be noted that the duct wall is rigid and its cross-section is assumed to be constant. Inside the duct, the flow is assumed to be uniform. Consequently, the duct modes are explicitly known.

Then, the finite/infinite element discretization of the weak formulation of the boundary value problem is performed, which allows for obtaining the computational model in the frequency domain. The unknowns of this computational model are the discretized acoustic velocity potential fields in the inner domain and in the outer domain, and the vector of the coefficients describing the unknown reflected sound field. In this computational model, the acoustic source term depends on the vector of the known coefficients of the incident sound field describing the fan acoustic excitation.

Finally, we introduce the frequency-dependent computational model that is a very large linear complex matrix system, which induces a large computational cost when exploring the frequency band of analysis. In addition, the nonparametric probabilistic approach of uncertainties (see [49, 50] and also [51]) has to be implemented in the computational model. For these two reasons, a computational reduced-order model depending on the frequency is constructed. For this purpose, two ingredients are introduced [46, 52]. The first one is a frequency by frequency static condensation of the aeroacoustic dofs on the liner dofs. Since the number of liner dofs is generally large, the reduced-order model is constructed by introducing a pellicular basis constituted of a set of acoustic modes in a pellicular domain that is related to liner dofs. All this methodology has been implemented in a commercial software [52-54], which are used for the numerical simulations presented in Section VII of the Paper.

\section{Definition of the geometry of the aeroacoustic problem}

For liner performance assessment, an aeroacoustic modeling of the acoustic radiation problem of a nacelle intake is used. The boundary value problem associated with this model (see Fig. 1) is formulated using three domains. In Fig. 1. a first domain $\Omega_{\mathrm{i}}$ corresponds to the nacelle near-field, which is discretized by finite elements. A second domain $\Omega_{\mathrm{o}}$ corresponds to the far-field, which is discretized by infinite elements. A third domain $\Omega_{\text {duct }}$ allows for analytically calculating the acoustic excitation imposed by the fan, for which the model is based on analytical duct modes ([3]). In order to numerically solve the intake acoustic radiation problem, the associated boundary value problem must be written involving a propagation equation and boundary conditions. For a given flight condition, the flow computation assumes that the fluid is inviscid (viscous and thermal effects are neglected) and is performed by solving the potential Euler equations on an appropriate CFD mesh. From these CFD computation, it can be deduced the steady-flow velocity field $\mathbf{v}_{0}$ and the steady-flow mass density field $\rho_{0}$. The convected acoustic wave equation comes from the derivation of the Navier-Stokes equations ([3], [6]), for which it is assumed an irrotational locally isentropic flow. The linearized convected acoustic equation is written in the frequency domain (assuming that fluctuating thermodynamic quantities are 
small compared to their mean-flow counterpart).



Fig. 1 2D section of the half-3D geometry for the boundary value problem of the intake radiation problem in presence of a flow, corresponding to the nacelle scheme in Fig 1 of the paper.

Fig. 1 1 is a $2 \mathrm{D}$ section of the half-3D geometry of the inlet acoustic problem, in which $\Omega_{\mathrm{i}}$ is the inner bounded open domain of $\mathbb{R}^{3}$ with boundary $\partial \Omega_{\mathrm{i}}=\Gamma_{\mathrm{io}} \cup \Gamma_{\mathrm{h}} \cup \Gamma \cup \Gamma_{\mathrm{f}}$ and where $\Omega_{\mathrm{o}}=\mathbb{R}^{3} \backslash \bar{\Omega}_{\mathrm{i}}$ is the outer unbounded open domain of $\mathbb{R}^{3}$, whose boundary is $\partial \Omega_{0}$. The generic point in $\mathbb{R}^{3}$ is denoted as $\mathbf{r}=(x, y, z)$, for which the direction of coordinate $z$ coincides with the rotation axis of the fan. The uniform inflow is in $z$-direction.

Definition of the geometry and boundary conditions for inner domain $\Omega_{\mathrm{i}}$. The unit normal to boundary $\partial \Omega_{\mathrm{i}}$ outward of domain $\Omega_{\mathrm{i}}$ is written as $\mathbf{n}_{\mathrm{io}}$ for the part $\Gamma_{\mathrm{io}}, \mathbf{n}_{\mathrm{h}}$ for the part $\Gamma_{\mathrm{h}}, \mathbf{n}$ for the part $\Gamma$, and $\mathbf{n}_{\mathrm{f}}$ for the part $\Gamma_{\mathrm{f}}$. The boundary part $\Gamma$ corresponds to the coupling interface between $\Omega_{\mathrm{i}}$ and the liner, while $\Gamma_{\mathrm{f}}$ is the boundary part on which the fan excitation is applied. The inner problem implies the use of the following boundary conditions:

- $\Gamma_{\text {io }}$ (inner outer) represents a nonphysical interface between inner and outer domains, on which the continuity condition is written for the acoustic velocity potential and for its gradient.

- $\Gamma_{\mathrm{h}}$ (hardwall) corresponds to rigid or "hardwall" part on which a zero normal acoustic velocity is applied.

- $\Gamma$ (liner) corresponds to the acoustic treatment characterized by its impedance.

- $\Gamma_{f}$ (fan) corresponds to the part of the boundary on which act acoustic sources induced by the fan.

Definition of the geometry and boundary conditions for outer domain $\Omega_{\mathbf{0}}$. The boundary $\partial \Omega_{\mathrm{o}}$ of $\Omega_{\mathrm{o}}$ is written as $\Gamma_{\text {io }} \cup \Gamma_{\text {ho }}$ in which $\Gamma_{\text {ho }}=\Gamma_{\mathrm{h}} \cup \Gamma \cup \Gamma_{\mathrm{f}}$ (this means that $\partial \Omega_{\mathrm{o}}=\partial \Omega_{\mathrm{i}}$ ). The unit normal to boundary $\partial \Omega_{\mathrm{o}}$ outward of domain $\Omega_{\mathrm{o}}$ is written as $\mathbf{n}_{\mathrm{oi}}=-\mathbf{n}_{\mathrm{io}}$ for the part $\Gamma_{\mathrm{io}}$ and $\mathbf{n}_{\mathrm{ho}}$ for the part $\Gamma_{\mathrm{ho}}$. For the acoustic wave propagation in the outer domain, $\Gamma_{\mathrm{ho}}$ corresponds to a rigid wall on which the normal acoustic velocity will be equal to zero.

The outer problem implies the use of the following boundary conditions:

- $\Gamma_{\text {io }}$ (inner outer) is the nonphysical coupling interface previously defined on which the continuity is written for the acoustic velocity potential and for its gradient (note that infinite boundary elements will be used on $\Gamma_{\text {io }}$ for taking into account the effects of $\Omega_{\mathrm{o}}$ on $\Omega_{\mathrm{i}}$ through the coupling interface $\Gamma_{\mathrm{io}}$ ).

- $\Gamma_{\text {ho }}$ (hardwall outer) corresponds to a rigid wall characterized by a zero normal acoustic velocity.

Definition of the geometry and boundary conditions for the semi-infinite duct $\Omega_{\text {duct }}$. A semi-infinite cylindrical duct $\Omega_{\text {duct }}$ is connected to the inner domain $\Omega_{\mathrm{i}}$ for which the coupling interface is the fan plane $\Gamma_{f}$. The boundary $\partial \Omega_{\text {duct }}$ of $\Omega_{\text {duct }}$ is written as $\partial \Omega_{\text {duct }}=\Gamma_{f} \cup \Gamma_{\text {duct }}$ in which $\Gamma_{\text {duct }}$ is the cylindrical-surface part of the boundary of $\Omega_{\text {duct }}$. It should be noted that this domain is only introduced for generating the acoustic excitation induced by the fan and consequently, has to be viewed as a "fictional domain" with respect to the formulation of the boundary value problem related to $\overline{\Omega_{\mathrm{i}} \cup \Omega_{\mathrm{o}}}$. This is the reason why $\Omega_{\mathrm{o}}=\mathbb{R}^{3} \backslash \bar{\Omega}_{\mathrm{i}}$. In this semi-infinite duct, the sound field is the sum of a 
given incident sound field and an unknown reflected sound field, which will be represented on the duct modes (see Section III.D.3). The wall of the boundary of $\Omega_{\text {duct }}$ is rigid on which a zero normal acoustic velocity is applied.

\section{Boundary value problem associated with the inner, outer, and duct domains of the intake acoustic radiation problem}

\section{Boundary value problem in the inner domain $\Omega_{i}$ (near-field)}

The definitions and the notations introduced in Section I.C are used.

Linear convected Helmholtz equation in $\Omega_{\mathbf{i}}$. The linear frequency formulation of the convected wave equation can be written $([1,4,6,16])$ as

$$
-\frac{\omega^{2} \rho_{0}}{c^{2}} \psi_{\mathrm{i}}+\frac{j \omega \rho_{0}}{c^{2}} \mathbf{v}_{0} \cdot \boldsymbol{\nabla} \psi_{\mathrm{i}}+\boldsymbol{\nabla} \cdot\left(\frac{j \omega \rho_{0} \mathbf{v}_{0}}{c^{2}} \psi_{\mathrm{i}}+\frac{\rho_{0} \mathbf{v}_{0}}{c^{2}} \mathbf{v}_{0} \cdot \boldsymbol{\nabla} \psi_{\mathrm{i}}-\rho_{0} \boldsymbol{\nabla} \psi_{\mathrm{i}}\right)=0 \quad \text { in } \quad \Omega_{\mathrm{i}},
$$

in which $\psi_{\mathrm{i}}$ is the acoustic velocity potential field in inner domain $\Omega_{\mathrm{i}}$, where $\mathbf{v}_{0}$ is the velocity field of the steady-flow, and where $\rho_{0}$ is the fluid mass density for the steady flow. The acoustic pressure field $p$ and the acoustic velocity field $\mathbf{v}$ are given by

$$
\begin{aligned}
& p=-j \omega \rho_{0} \psi_{\mathrm{i}}-\rho_{0} \mathbf{v}_{0} \cdot \boldsymbol{\nabla} \psi_{\mathrm{i}} \text { in } \Omega_{\mathrm{i}}, \\
& \mathbf{v}=\boldsymbol{\nabla} \psi_{\mathrm{i}} \text { in } \Omega_{\mathrm{i}} .
\end{aligned}
$$

In the above equations, $c$ is the speed of sound at equilibrium, $\omega$ is the angular frequency $(\mathrm{rad} / \mathrm{s}), j=\sqrt{-1}$ is the imaginary unit complex number, $\boldsymbol{\nabla}$ is the gradient operator with respect to space variable $\mathbf{r}=(x, y, z)$, and where the symbol "dot" represents the Euclidean inner product in $\mathbb{R}^{3}$.

Boundary conditions on $\partial \Omega_{\mathbf{i}}=\Gamma_{\mathbf{i o}} \cup \Gamma_{\mathbf{h}} \cup \Gamma \cup \Gamma_{\mathbf{f}}$. The boundary conditions on the parts of $\Omega_{\mathrm{i}}$ are

$$
\begin{array}{ll}
\psi_{\mathrm{i}}=\psi_{\mathrm{o}}, & \boldsymbol{\nabla} \psi_{\mathrm{i}}=\boldsymbol{\nabla} \psi_{\mathrm{o}} \quad \text { on } \quad \Gamma_{\mathrm{io}}, \\
\frac{\partial \psi_{\mathrm{i}}}{\partial \mathbf{n}_{\mathrm{h}}}=0 & \text { on } \quad \Gamma_{\mathrm{h}}, \\
\psi_{\mathrm{i}}=\varphi & , \quad \boldsymbol{\nabla} \psi_{\mathrm{i}}=\boldsymbol{\nabla} \varphi \quad \text { on } \quad \Gamma_{f},
\end{array}
$$

in which $\psi_{\mathrm{o}}$ is the acoustic velocity potential field in outer domain $\Omega_{\mathrm{o}}$ and where $\varphi$ is the acoustic velocity potential field in $\Omega_{\text {duct }}$, which will have to satisfy the boundary value problem in $\Omega_{\text {duct }}$, detailed in Section II.D.3 The boundary condition on $\Gamma$ (liner) is written $([47],[22])$ as

$$
\frac{\partial \psi_{i}}{\partial \mathbf{n}}=\left(1-\frac{1}{j \omega} \mathbf{v}_{0} \cdot \boldsymbol{\nabla}+\frac{1}{j \omega} \mathbf{n} \cdot(\mathbf{n} \cdot \boldsymbol{\nabla}) \mathbf{v}_{0}\right)\left(\frac{1}{Z(\mathbf{r}, \omega)}\left(j \omega \rho_{0} \psi_{\mathrm{i}}+\rho_{0} \mathbf{v}_{0} \cdot \boldsymbol{\nabla} \psi_{\mathrm{i}}\right)\right) \quad \text { on } \quad \Gamma,
$$

in which $Z(\mathbf{r}, \omega)^{-1}$ denotes the local admittance of the liner at point $\mathbf{r}$ and at frequency $\omega$.

\section{Boundary value problem in the outer domain $\Omega_{o}$}

Linear convected Helmholtz equation in $\Omega_{\mathbf{0}}$. Similarly to Eq. (1), the linear convected Helmholtz equation in $\Omega_{\mathrm{o}}$ is written as

$$
-\frac{\omega^{2} \rho_{\infty}}{c^{2}} \psi_{\mathrm{o}}+\frac{j \omega \rho_{\infty}}{c^{2}} \mathbf{v}_{\infty} \cdot \boldsymbol{\nabla} \psi_{\mathrm{o}}+\boldsymbol{\nabla} \cdot\left(\frac{j \omega \rho_{\infty} \mathbf{v}_{\infty}}{c^{2}} \psi_{\mathrm{o}}+\frac{\rho_{\infty} \mathbf{v}_{\infty}}{c^{2}} \mathbf{v}_{\infty} \cdot \boldsymbol{\nabla} \psi_{\mathrm{o}}-\rho_{\infty} \boldsymbol{\nabla} \psi_{\mathrm{o}}\right)=0 \quad \text { in } \quad \Omega_{\mathrm{o}},
$$

in which $\psi_{\mathrm{o}}$ is the acoustic velocity potential field in $\Omega_{\mathrm{o}}$, where $\rho_{\infty}$ and $\mathbf{v}_{\infty}$ are the constant mass density and the constant velocity field of the uniform inflow in z-direction. 
Boundary conditions on $\partial \Omega_{\mathbf{o}}=\Gamma_{\mathbf{i o}} \cup \Gamma_{\mathbf{h o}}$. The boundary conditions on the parts of $\partial \Omega_{\mathrm{o}}$ are

$$
\begin{gathered}
\psi_{\mathrm{o}}=\psi_{\mathrm{i}}, \quad \boldsymbol{\nabla} \psi_{\mathrm{o}}=\boldsymbol{\nabla} \psi_{\mathrm{i}} \quad \text { on } \Gamma_{\mathrm{io}}, \\
\frac{\partial \psi_{\mathrm{o}}}{\partial \mathbf{n}_{\mathrm{oi}}}=0 \text { on } \Gamma_{\mathrm{ho}}
\end{gathered}
$$

in which $\psi_{\mathrm{i}}$ is the acoustic velocity potential field in $\Omega_{\mathrm{i}}$. At infinity, the outward Sommerfeld radiation condition is written as

$$
\lim _{R \rightarrow+\infty}\left\{\frac{\partial \psi_{\mathrm{o}}}{\partial R}+j \frac{\omega}{c} \psi_{\mathrm{o}}+M_{\infty} \frac{\partial \psi_{\mathrm{o}}}{\partial R}\right\}=0
$$

in which $R=\|\mathbf{r}\|$, where $\partial / \partial R$ denotes the derivative in the radial direction from the origin, and where $M_{\infty}=\left\|\mathbf{v}_{\infty}\right\| / c$.

\section{Boundary value problem in the semi-infinite duct $\Omega_{\text {duct }}$ for the inlet case and duct modes}

For the fan excitation (see for instance [3] and [7]), the acoustic velocity potential field $\varphi$ in $\Omega_{\text {duct }}$ will be represented on the family of acoustic duct modes and will allow (i) for ensuring the coupling between the semi-infinite duct $\Omega_{\text {duct }}$ and the inner domain $\Omega_{\mathrm{i}}$ on boundary $\Gamma_{f}$ (see Fig. 1), and (ii) for representing the acoustic excitation produced by the fan. It should be noted that the duct modes are explicitly known for a simple cross-section of the duct such as a circle or a rectangle, and can be computed for a nonsimple geometry of the cross-section. In this work, it is assumed that $\Omega_{\text {duct }}$ is a straight cylinder with $z$-axis, for which its cross-section is constant and consequently, independent of $z$. The steady flow in $\Omega_{\text {duct }}$ is assumed to be subsonic and uniform along $z$-direction.

Boundary value problem in $\Omega_{\text {duct }}$. We consider the acoustic propagation in $z$-direction. The acoustic velocity potential field $(x, y, z) \mapsto \varphi(x, y, z)$ verifies the following linear convected Helmholtz equation,

$$
\Delta_{x y} \varphi+\left(1-M_{\infty}^{2}\right) \frac{\partial^{2} \varphi}{\partial z^{2}}-2 j k M_{\infty} \frac{\partial \varphi}{\partial z}+k^{2} \varphi=0 \text { in } \Omega_{\mathrm{duct}},
$$

where $\Delta_{x y}$ is the Laplacian in the transversal plane $(x, y), M_{\infty}=\left\|\mathbf{v}_{\infty}\right\| / c$ is the Mach number associated with the uniform, $k=\omega / c$ is the wave number. The boundary condition on $\Gamma_{\text {duct }}$ consists in writing that the normal derivative of the acoustic velocity potential is zero,

$$
\frac{\partial \varphi}{\partial \mathbf{n}_{\text {duct }}}=0 \text { on } \Gamma_{\text {duct }},
$$

where $\mathbf{n}_{\text {duct }}$ is the external unit normal to $\partial \Omega_{\text {duct }}$. In addition, the continuity on interface $\Gamma_{f}$ of acoustic velocity potential field $\varphi$ with acoustic velocity potential field $\psi_{\mathrm{i}}$ is

$$
\varphi=\psi_{\mathrm{i}} \quad, \quad \nabla \varphi=\nabla \psi_{\mathrm{i}} \quad \text { on } \quad \Gamma_{f}
$$

Duct modes and modal representation of the acoustic velocity potential field $\varphi$. Let $\mathcal{S}_{\text {duct }}$ be the constant crosssection in the $(x, y)$-plane (independent of $z$ ) of the straight cylindrical duct $\Omega_{\text {duct }}$. The solution of the boundary value problem defined by Eqs. (12), (13), and (14) can be written as

$$
\varphi(x, y, z)=\sum_{\alpha=1}^{\infty} c_{\alpha} \varphi_{\alpha}(x, y) e^{j k_{z \alpha} z}
$$

in which $(x, y) \mapsto \varphi_{\alpha}(x, y)$ is defined in $\mathcal{S}_{\text {duct }}$, where $k_{z_{\alpha}}$ is the axial wavenumber of the duct mode $\alpha$ (note that we have used the opposite of the harmonic wave convention previously introduced, it is $+j k_{z_{\alpha}} z$ and not $-j k_{z_{\alpha}} z$ ), and where $c_{\alpha}$ is the modal component of duct mode $\alpha$. Substituting Eq. (15) into Eq. (12), it can be seen that $\varphi_{\alpha}$ has to verify the following $2 \mathrm{D}$ wave equation in $\mathcal{S}_{\text {duct }}$, which is written as [55],

$$
\Delta_{x y} \varphi_{\alpha}(x, y)+k_{x y_{\alpha}}^{2} \varphi_{\alpha}(x, y)=0 \quad \text { in } \mathcal{S}_{\text {duct }} .
$$

The family of real-valued eigenfunctions $\left\{\varphi_{\alpha}\right\}_{\alpha}$ is such that,

$$
\int_{\mathcal{S}_{\text {duct }}} \varphi_{\alpha}(x, y) \varphi_{\beta}(x, y) d x d y=\delta_{\alpha \beta}
$$


where $\delta_{\alpha \beta}$ is the Kronecker symbol. The transversal wavenumber $k_{x y_{\alpha}}$ of a given duct mode $\alpha$ is related to the axial wavenumber $k_{z_{\alpha}}$ by the two following equations,

$$
k_{z_{\alpha}}=\frac{k M_{\infty} \pm \sqrt{k^{2}-\beta^{2} k_{x y_{\alpha}}^{2}}}{\beta^{2}},
$$

in which $\beta=\sqrt{1-M_{\infty}^{2}}$. The axial wavenumber $k_{z_{\alpha}}$ of a given mode $\alpha$ can be real (propagative mode) or purely imaginary (evanescent mode).

Modal representation for the acoustic excitation by the fan and the reflected sound field. The acoustic velocity potential field $\varphi$ defined by Eq. [15] is rewritten by keeping only a finite number $N_{d}$ of duct modes and by showing the contribution of the given incident sound field (represented by the symbol +) that represents the acoustic excitation induced by the fan and the contribution of the unknown associated reflected sound field (represented by the symbol -). From Eqs. (15) and (18), function $\varphi(x, y, z)$ can be rewritten as

$$
\varphi(x, y, z)=\sum_{\alpha=1}^{N_{d}} \varphi_{\alpha}(x, y)\left(c_{\alpha}^{+} e^{j k_{z \alpha}^{+} z}+c_{\alpha}^{-} e^{j k_{z_{\alpha}}^{-} z}\right),
$$

in which $k_{z_{\alpha}}^{+}$is the axial wavenumber of the wave propagating in the upstream direction and $k_{z_{\alpha}}^{-}$is the axial wavenumber of the wave propagating in the downstream direction, which are expressed using Eq. (18) as

$$
k_{z_{\alpha}}^{ \pm}=\frac{k M_{\infty} \pm \gamma_{\alpha}}{\beta^{2}},
$$

where $\gamma_{\alpha}=\sqrt{k^{2}-\beta^{2} k_{x y_{\alpha}}^{2}}$.

It should be noted that the acoustic excitation induced by the fan, which is associated with the incident sound field, is defined by giving the complex vector $\mathbf{c}^{+}=\left(c_{1}^{+}, \ldots, c_{N_{d}}^{+}\right)$, while the unknown reflected sound field depends on the vector $\mathbf{c}^{-}=\left(c_{1}^{-}, \ldots, c_{N_{d}}^{-}\right)$and will be included with all the unknown variables of the acoustic problem. For describing the acoustic excitation of the fan, $\mathbf{c}^{+}$can be chosen as dependent on $\omega$ and will then be denoted as $\mathbf{c}^{+}(\omega)$. On the other hand, whether or not $\mathbf{c}^{+}$depends on $\omega$, as $\mathbf{c}^{-}$is an unknown of the problem, $\mathbf{c}^{-}$always depends on $\omega$, which we will denote by $\mathbf{c}^{-}(\omega)$.

\section{E. Finite/Infinite Element discretization of the weak formulation}

The weak formulation of the boundary value problem defined by Eqs. (1) to (11) with $\varphi$ given by (19) is discretized by the Finite/Infinite Element Method (FEM/IEM). For all $\omega$ fixed in $\mathbb{R}$, let $\psi_{\mathrm{i}}(\omega)$ be the complex vector in $\mathbb{C}^{N_{\mathrm{i}}}$ corresponding to the finite element discretization of field $\psi_{\mathrm{i}}(\omega)$ in which $N_{\mathrm{i}}$ is the number of degrees of freedom (dofs). Let $\psi_{\mathrm{o}}(\omega)$ be the complex vector in $\mathbb{C}^{N_{\mathrm{o}}}$ corresponding to the finite/infinite element discretization of field $\psi_{\mathrm{o}}(\omega)$ in which $N_{\mathrm{o}}$ is the number of dofs. We then obtain the computational model in terms of $\psi_{\mathrm{i}}(\omega), \psi_{\mathrm{o}}(\omega)$, and $\mathbf{c}^{-}(\omega)$, which is written as

$$
\left[\begin{array}{ccc}
{\left[A_{\mathrm{i}}(\omega)\right]+\left[Z_{\mathrm{i}}(\omega)\right]} & {[H(\omega)]} & -\left[\mathcal{E}_{\mathrm{if}}\right]\left[F^{-}(\omega)\right] \\
-[H(\omega)]^{T} & {\left[A_{\mathrm{o}}(\omega)\right]} & {[0]} \\
{[E]} & {[0]} & -\left[R^{-}(\omega)\right]
\end{array}\right]\left[\begin{array}{l}
\boldsymbol{\psi}_{\mathrm{i}}(\omega) \\
\boldsymbol{\psi}_{\mathrm{o}}(\omega) \\
\mathbf{c}^{-}(\omega)
\end{array}\right]=\left[\begin{array}{c}
{\left[\mathcal{E}_{\mathrm{if}}\right]\left[F^{+}(\omega)\right]} \\
{[0]} \\
{\left[R^{+}(\omega)\right]}
\end{array}\right] \mathbf{c}^{+}(\omega)
$$

in which,

- the $\left(N_{\mathrm{i}} \times N_{\mathrm{i}}\right)$ complex matrix $\left[A_{\mathrm{i}}(\omega)\right]$, the $\left(N_{\mathrm{o}} \times N_{\mathrm{o}}\right)$ complex matrix $\left[A_{\mathrm{o}}(\omega)\right]$, and the $\left(N_{\mathrm{i}} \times N_{\mathrm{o}}\right)$ complex matrix $[H(\omega)]$ correspond to the block decomposition of the aeroacoustic impedance matrix.

- the diagonal $\left(N_{\mathrm{d}} \times N_{\mathrm{d}}\right)$ complex matrices $\left[R^{+}(\omega)\right]$ and $\left[R^{-}(\omega)\right]$ correspond to the incident and to the reflected modal participations in the duct domain $\Omega_{\text {duct }}$.

- the $\left(N_{\mathrm{d}} \times N_{\mathrm{i}}\right)$ constant real matrix [E] corresponds to the finite element discretization of the inner product in the space of all the square integrable functions on boundary $\Gamma_{\mathrm{f}}$.

- the $\left(N_{\mathrm{i}} \times N_{\mathrm{f}}\right)$ real matrix [ $\left.\mathcal{E}_{\mathrm{if}}\right]$ constituted of ones and zeros, allows for selecting the dofs related to boundary $\Gamma_{\mathrm{f}}$.

- the $\left(N_{\mathrm{f}} \times N_{\mathrm{d}}\right)$ complex matrices $\left[F^{+}(\omega)\right]$ and $\left[F^{-}(\omega)\right]$ represent the discretization of the incident and the reflected modal participations in the inner domain $\Omega_{\mathrm{i}}$. 


\section{Computational reduced-order model}

\section{A. Frequency by frequency static condensation with respect to the liner dofs and expression of the fan acoustic excitation}

In Section IV] we will present the probabilistic modeling of uncertainties in the computational model defined by Eq. 21). These uncertainties will be taken into account for both the parametric uncertainties and the model uncertainties induced by the modeling errors for the aeroacoustic and for the liner. Consequently, we need to reorganize the algebraic structure of the complex matrix equation defined by Eq. 21 in order to exhibit the complex vector $\psi_{\ell}(\omega)$ belonging to $\mathbb{C}^{N_{\ell}}$ of the $N_{\ell}$ dofs of the liner and the complex vector $\boldsymbol{\psi}_{a}$ belonging to $\mathbb{C}^{N_{a}}$ of the $N_{a}$ other dofs of $\left(\boldsymbol{\psi}_{\mathrm{i}}(\omega), \boldsymbol{\psi}_{\mathrm{o}}(\omega), \mathbf{c}^{-}(\omega)\right)$. This means that $N_{a}=N_{\mathrm{i}}+N_{\mathrm{o}}+N_{d}-N_{\ell}$ and that,

$$
\left[\begin{array}{l}
\boldsymbol{\psi}_{\mathrm{i}}(\omega) \\
\boldsymbol{\psi}_{\mathrm{o}}(\omega) \\
\mathbf{c}^{-}(\omega)
\end{array}\right] \text { is rewritten as }\left[\begin{array}{l}
\boldsymbol{\psi}_{\ell}(\omega) \\
\boldsymbol{\psi}_{a}(\omega)
\end{array}\right], \quad N_{\mathrm{i}}+N_{\mathrm{o}}+N_{d}=N_{\ell}+N_{a} .
$$

Since $\Gamma \cap \Gamma_{\mathrm{f}}=\{\emptyset\}$, we have

$$
\left[\begin{array}{c}
{\left[\mathcal{E}_{\mathrm{if}}\right]\left[F^{+}(\omega)\right]} \\
{[0]} \\
{\left[R^{+}(\omega)\right]}
\end{array}\right] \mathbf{c}^{+}(\omega) \text { is rewritten as }\left[\begin{array}{c}
\mathbf{0} \\
\mathbf{f}_{a}(\omega)
\end{array}\right] \quad, \quad N_{\mathrm{i}}+N_{\mathrm{o}}+N_{d}=N_{\ell}+N_{a},
$$

in which the $\mathbb{C}^{N_{a}}$-vector $\mathbf{f}_{a}(\omega)$ can be written as

$$
\mathbf{f}_{a}(\omega)=\left[\mathcal{B}_{a}(\omega)\right] \mathbf{c}^{+}(\omega),
$$

where $\left[\mathcal{B}_{a}(\omega)\right]$ is a known complex $\left(N_{a} \times N_{d}\right)$ matrix. Using Eqs. (22) and 23), the computational model defined by Eq. (21) can be rewritten in a block form as

$$
\left[\begin{array}{ll}
{\left[A_{\ell \ell}(\omega)\right]} & {\left[A_{\ell a}(\omega)\right]} \\
{\left[A_{a \ell}(\omega)\right]} & {\left[A_{a a}(\omega)\right]}
\end{array}\right]\left[\begin{array}{l}
\psi_{\ell}(\omega) \\
\psi_{a}(\omega)
\end{array}\right]+\left[\begin{array}{cc}
{\left[Z_{\ell}(\omega)\right]} & 0 \\
0 & 0
\end{array}\right]\left[\begin{array}{l}
\psi_{\ell}(\omega) \\
\psi_{a}(\omega)
\end{array}\right]=\left[\begin{array}{c}
\mathbf{0} \\
\mathbf{f}_{a}(\omega)
\end{array}\right]
$$

For solving Eq. [25], the following $\omega$ by $\omega$ static condensation (Schur complement) is performed. It consists in eliminating the vector $\mathbf{x}_{a}$ in the following matrix equation,

$$
\left[\begin{array}{ll}
{\left[A_{\ell \ell}(\omega)\right]} & {\left[A_{\ell a}(\omega)\right]} \\
{\left[A_{a \ell}(\omega)\right]} & {\left[A_{a a}(\omega)\right]}
\end{array}\right]\left[\begin{array}{l}
\mathbf{x}_{\ell} \\
\mathbf{x}_{a}
\end{array}\right]=\left[\begin{array}{c}
\mathbf{0} \\
\mathbf{f}_{a}(\omega)
\end{array}\right]
$$

which yields $\left[\mathcal{A}_{\ell}(\omega)\right] \mathbf{x}_{\ell}=\mathcal{F}_{\ell}(\omega)$, in which the $\left(N_{\ell} \times N_{\ell}\right)$ complex matrix $\left[\mathcal{A}_{\ell}(\omega)\right]$ is defined by

$$
\left[\mathcal{A}_{\ell}(\omega)\right]=\left[A_{\ell \ell}(\omega)\right]-\left[A_{\ell a}(\omega)\right]\left[A_{a a}(\omega)\right]^{-1}\left[A_{a \ell}(\omega)\right],
$$

and where the $N_{\ell}$ complex vector $\mathcal{F}_{\ell}(\omega)$ is written as $\mathcal{F}_{\ell}(\omega)=-\left[A_{\ell a}(\omega)\right]\left[A_{a a}(\omega)\right]^{-1} \mathbf{f}_{a}(\omega)$, which can be rewritten, using Eq. [24], as $\mathcal{F}_{\ell}(\omega)=\left[\mathcal{B}_{\ell}(\omega)\right] \mathbf{c}^{+}(\omega)$ in which the $\left(N_{\ell} \times N_{d}\right)$ complex matrix $\left[\mathcal{B}_{\ell}(\omega)\right]$ is defined by

$$
\left[\mathcal{B}_{\ell}(\omega)\right]=-\left[A_{\ell a}(\omega)\right]\left[A_{a a}(\omega)\right]^{-1}\left[\mathcal{B}_{a}(\omega)\right] .
$$

Using this $\omega$ by $\omega$ static condensation, the elimination of $\psi_{a}(\omega)$ in Eq. 25) yields,

$$
\left(\left[\mathcal{A}_{\ell}(\omega)\right]+\left[Z_{\ell}(\omega)\right]\right) \psi_{\ell}(\omega)=\left[\mathcal{B}_{\ell}(\omega)\right] \mathbf{c}^{+}(\omega) .
$$

\section{B. Computational reduced-order model}

Following the explanations given at the beginning of Section III.A the uncertainties will be taken into account by using the nonparametric probabilistic approach [50]. Such an approach requires the introduction of a reduced-order model associated with Eq 29]. For such a construction we need to introduce a reduced-order basis that is chosen as a pellicular basis (see [52, 56]) whose construction is summarized hereinafter. 


\section{Construction of the pellicular basis}

This pellicular basis is associated with the acoustic radiation of the liner delimited by its boundary $\Gamma$ on which a thin acoustic layer (the pellicular domain) is introduced. Considering that the acoustic layer thickness is infinitely thin regarding to the acoustic wavelength, a set of acoustic modes related to the pellicular domain is computed solving the eigenvalue problem derived from the computational model, $\left[\mathcal{K}_{L}\right] \phi_{\alpha}=\lambda_{\alpha}\left[\mathcal{M}_{L}\right] \phi_{\alpha}$, in which the symmetric $\left(N_{\ell} \times N_{\ell}\right)$ real matrices $\left[\mathcal{K}_{L}\right]$ and $\left[\mathcal{M}_{L}\right]$ are positive definite and represent, respectively, the acoustic stiffness matrix and the associated mass matrix. The eigenvector $\phi_{\alpha}$ belonging to $\mathbb{R}^{N_{\ell}}$ is called a pellicular eigenvector and $\lambda_{\alpha}$ is its corresponding eigenvalue. These pellicular eigenvectors verify the following orthogonal properties, $\boldsymbol{\phi}_{\alpha}^{T}\left[\mathcal{K}_{L}\right] \boldsymbol{\phi}_{\beta}=\lambda_{\alpha} \delta_{\alpha \beta}$ and $\boldsymbol{\phi}_{\alpha}^{T}\left[\mathcal{M}_{L}\right] \boldsymbol{\phi}_{\beta}=\delta_{\alpha \beta}$ in which $\delta_{\alpha \beta}$ is the Kronecker symbol. Let $n_{\ell}<N_{\ell}$ be the number of pellicular eigenvectors $\phi_{1}, \ldots, \phi_{n_{\ell}}$ that are retained for the construction of the reduced-order model. We then introduce the $\left(N_{\ell} \times n_{\ell}\right)$ real matrix $[\Phi]$ whose columns are $\phi_{1}, \ldots, \phi_{n_{\ell}}$.

\section{Construction of the computational reduced-order model}

The computational reduced-order model is obtained by projecting Eq. 29] on the subspace spanned by [Ф] and is thus written as

$$
\begin{gathered}
\boldsymbol{\psi}_{\ell}(\omega)=[\Phi] \mathbf{q}(\omega), \\
([\mathbb{A}(\omega)]+[\mathbb{Z}(\omega)]) \mathbf{q}(\omega)=[\mathbb{B}(\omega)] \mathbf{c}^{+}(\omega),
\end{gathered}
$$

in which $\mathbf{q}(\omega)$, which belongs to $\mathbb{C}^{n_{\ell}}$, is a complex vector of the generalized coordinates, and where the $\left(n_{\ell} \times n_{\ell}\right)$ complex matrix $[\mathbb{A}(\omega)]$, the $\left(n_{\ell} \times n_{\ell}\right)$ complex matrix $[\mathbb{Z}(\omega)]$, and the $\left(n_{\ell} \times N_{d}\right)$ complex matrix $[\mathbb{B}(\omega)]$ are such that

$$
\begin{aligned}
{[\mathbb{A}(\omega)] } & =[\Phi]^{T}\left[\mathcal{A}_{\ell}(\omega)\right][\Phi], \\
{[\mathbb{Z}(\omega)] } & =[\Phi]^{T}\left[Z_{\ell}(\omega)\right][\Phi], \\
{[\mathbb{B}(\omega)] } & =[\Phi]^{T}\left[\mathcal{B}_{\ell}(\omega)\right] .
\end{aligned}
$$

Once $\psi_{\ell}(\omega)$ is known, $\psi_{a}(\omega)$ is computed by using the second line of Eq. 25) in which $\mathbf{f}_{a}(\omega)$ is substituted by its expression given by Eq. 24,

$$
\left[A_{a a}(\omega)\right] \psi_{a}(\omega)=\left[\mathcal{B}_{a}(\omega)\right] \mathbf{c}^{+}(\omega)-\left[A_{a \ell}(\omega)\right] \psi_{\ell}(\omega) .
$$

We introduce a vector $\mathbf{q o I}(\omega)$ of quantities of interest related to the far-field acoustic pressure at several given spatial points. This vector can always be written as

$$
\mathbf{q o I}(\omega)=\left[\mathbb{O}_{\mathrm{obs}}(\omega)\right] \psi_{a}(\omega),
$$

in which $\left[\mathrm{O}_{\mathrm{obs}}(\omega)\right]$ is a given observation matrix. Combining Eqs. (30) and (35) shows that Eq. (36) can be rewritten as

$$
\mathbf{q o I}(\omega)=\left[\mathbb{F}_{\mathrm{qoI}}(\omega)\right] \mathbf{c}^{+}(\omega)-\left[\mathbb{A}_{\mathrm{qoI}}(\omega)\right] \mathbf{q}(\omega),
$$

in which the matrices $\left[\mathbb{F}_{\text {qoI }}(\omega)\right]$ and $\left[\mathbb{A}_{\mathrm{qoI}}(\omega)\right]$ are formally given by $\left[\mathbb{F}_{\mathrm{qoI}}(\omega)\right]=\left[\mathcal{O}_{\mathrm{obs}}(\omega)\right]\left[A_{a a}(\omega)\right]^{-1}\left[\mathcal{B}_{a}(\omega)\right]$ and $\left[\mathbb{A}_{\mathrm{qoI}}(\omega)\right]=\left[\mathbb{O}_{\mathrm{obs}}(\omega)\right]\left[A_{a a}(\omega)\right]^{-1}\left[A_{a \ell}(\omega)\right][\Phi]$.

In the framework of the nonparametric probabilistic approach, the uncertainties in the computational model are implemented by substituting complex matrices $[\mathbb{A}(\omega)]$ and $[\mathbb{Z}(\omega)]$ by random complex matrices $[\mathbf{A}(\omega)]$ and $[\mathbf{Z}(\omega)]$ in Eq. (31). The uncertainties on vector $\mathbf{c}^{+}(\omega)$ are taken into account by the parametric probabilistic, which consists in modeling $\mathbf{c}^{+}(\omega)$ by a random vector $\mathbf{C}^{+}(\omega)$. In order to implement the nonparametric probabilistic approach, we need to introduce the algebraic properties of matrices $[\mathbb{A}(\omega)]$ and $[\mathbb{Z}(\omega)]$, which are defined in Sections III.C and III.D

\section{Algebraic properties of complex matrix $[\mathbb{Z}(\omega)]$ related to the liner impedance}

The complex matrix $[\mathbb{Z}(\omega)]$ defined by Eq. 33 is written as $[\mathbb{Z}(\omega)]=\left[\mathbb{Z}_{R}(\omega)\right]+j\left[\mathbb{Z}_{I}(\omega)\right]$, where $\left[\mathbb{Z}_{R}(\omega)\right]=\operatorname{Re}\{[\mathbb{Z}(\omega)]\}$ and $\left[\mathbb{Z}_{I}(\omega)\right]=\operatorname{Im}\{[\mathbb{Z}(\omega)]\}$ are nonsymmetric real matrices (the symmetry only appears for $\mathbf{v}_{0}=\mathbf{0}$, that is to say without flow). The analysis of the formulation presented shows that the imaginary part of matrix $[\mathbb{Z}(\omega)]$, that is to say the real 
matrix $\left[\mathbb{Z}_{I}(\omega)\right]$, corresponds to a dissipative term due to the liner. As $[\mathbb{Z}(\omega)] \neq[\mathbb{Z}(\omega)]^{T}$ (nonsymmetric matrix for $\left.\mathbf{v}_{0} \neq \mathbf{0}\right)$, the dissipative part is generated by the symmetric part $\left[\mathbb{Z}_{I}^{\mathrm{S}}(\omega)\right]$ of $\left[\mathbb{Z}_{I}(\omega)\right]$, the skew-symmetric part $\left[\mathbb{Z}_{I}^{\text {SS }}(\omega)\right]$ of $\left[\mathbb{Z}_{I}(\omega)\right]$ does not dissipate energy. Consequently, the real matrix $\left[\mathbb{Z}_{I}(\omega)\right]$ is written as $\left[\mathbb{Z}_{I}(\omega)\right]=\left[\mathbb{Z}_{I}^{S}(\omega)\right]+\left[\mathbb{Z}_{I}^{\text {SS }}(\omega)\right]$, in which

$$
\left[\mathbb{Z}_{I}^{\mathrm{S}}(\omega)\right]=\frac{1}{2}\left(\left[\mathbb{Z}_{I}(\omega)\right]+\left[\mathbb{Z}_{I}(\omega)\right]^{T}\right)
$$

and where $\left[\mathbb{Z}_{I}^{\mathrm{SS}}(\omega)\right]=\left(\left[\mathbb{Z}_{I}(\omega)\right]-\left[\mathbb{Z}_{I}(\omega)\right]^{T}\right) / 2$. It can be viewed that the positive-definite symmetric real matrix $\left[\mathbb{Z}_{I}^{\mathrm{S}}(\omega)\right]$ is associated with the bilinear form in $\psi_{i}$ and $\delta \psi_{i}$,

$$
\int_{\Gamma} \frac{\rho_{0}^{2} Z_{R}(\mathbf{r}, \omega)}{\omega\left(Z_{R}^{2}(\mathbf{r}, \omega)+Z_{I}^{2}(\mathbf{r}, \omega)\right)}\left(\omega^{2} \psi_{i} \delta \psi_{i}+\left(\mathbf{v}_{0} \cdot \nabla \psi_{i}\right)\left(\mathbf{v}_{0} \cdot \nabla \delta \psi_{i}\right)\right) d s(\mathbf{r}),
$$

which is effectively a positive-definite symmetric bilinear form because $\omega>0$ and the local resistance $Z_{R}(\mathbf{r}, \omega)$ is positive (by convention). Finally, matrix $[\mathbb{Z}(\omega)]$ defined by Eq. 33) can be rewritten as

$$
[\mathbb{Z}(\omega)]=\left[\mathbb{Z}^{\mathrm{NS}}(\omega)\right]+j\left[\mathbb{Z}_{I}^{\mathrm{S}}(\omega)\right],
$$

in which $\left[\mathbb{Z}_{I}^{S}(\omega)\right]$ is a positive-definite symmetric real matrix corresponding to a dissipative term due to the liner in the coupled equation defined by Eq. 31] and where $\left[\mathbb{Z}^{\mathrm{NS}}(\omega)\right]$ is a complex matrix that is not symmetric (and not skew-symmetric), which is written as

$$
\left[\mathbb{Z}^{\mathrm{NS}}(\omega)\right]=\left[\mathbb{Z}_{R}(\omega)\right]+j\left[\mathbb{Z}_{I}^{\mathrm{SS}}(\omega)\right]
$$

\section{Algebraic properties of complex matrix $[\mathbb{A}(\omega)]$ related to the aeroacoustic impedance}

In Eq. [31], the reduced complex matrix $[\mathbb{A}(\omega)]$, related to aeroacoustics, is defined by Eq. (32) and could be written as $[\mathbb{A}(\omega)]=\left[\mathbb{A}_{R}(\omega)\right]+j\left[\mathbb{A}_{I}(\omega)\right]$, in which $\left[\mathbb{A}_{R}(\omega)\right]=\operatorname{Re}\{[\mathbb{A}(\omega)]\}$ and $\left[\mathbb{A}_{I}(\omega)\right]=\operatorname{Im}\{[\mathbb{A}(\omega)]\}$ are nonsymmetric real matrices (due to the flow $\mathbf{v}_{0} \neq \mathbf{0}$ ). For the inlet case, there is an acoustic radiation of the outgoing acoustic fields at infinity (Sommerfeld condition in the outer domain $\Omega_{r m o}$ and in the duct domain $\Omega_{\text {duct }}$ ), which induces an apparent dissipation. Unfortunately, due to the presence of the flow, the method presented in Section [III.C for extracting the dissipation part in the complex matrix $[\mathbb{Z}(\omega)]$ cannot be used for the aeroacoustic complex matrix $[\mathbb{A}(\omega)]$ in the framework of the generalized formulation available in Actran/TM. Consequently, because the dissipation part cannot be extracted, the stochastic modeling of the aeroacoustic matrix will globally be carried out as presented in Section IV. without separating the real part and the imaginary part.

\section{Probabilistic model for uncertainty quantification}

As previously explained, three sources of uncertainties are taken into account. The first one is related to uncertainties induced by modeling errors in the impedance matrix of the liner appearing in the computational reduced-order model. The second one corresponds to model uncertainties in the aeroacoustic part of the computational model including the mean-flow computation (CFD), the acoustic equations in moving fluids, and the duct acoustic related to the fan excitation. The third one is related to uncertainties in the acoustic excitation induced by the fan. The stochastic solver of the random equation is presented. For each value of the frequency, the Stochastic Reduced-Order Model (SROM) is solved by the Monte Carlo numerical simulation method [57]. The convergence analysis is carried out with respect to the number of realizations. For the first two sources of uncertainties, the nonparametric probabilistic approach is used, in particular the construction of the probabilistic model of the random matrices (see [50]). For the third one, the parametric probabilistic approach is used.

\section{A. Brief summary of the nonparametric probabilistic approach of uncertainties}

For a given flight condition and given environment, the boundary value problem considered is that of aeroacoustics whose equations are those of the linearized convected acoustic wave equations formulated in the frequency domain. This aeroacoustic boundary problem depends on (i) the geometry of the very complex and unbounded domain, (ii) the boundary conditions, in particular those related to the impedance of the liner that is an elastoacoustic system, (iii) the use of the linearized convected Helmholtz equation instead of the Navier-Stokes equations, (iv) the physical 
system parameters, (v) the velocity field of the flow, which is calculated by the CFD for a given flight condition, and (vi) the acoustic excitation induced by the fan. The development of the aeroacoustic computational model for this boundary value problem requires the introduction of simplifications and approximations: simplification of the geometry, simplification of the model describing the acoustic excitation of the fan, simplification of the internal geometry of the reactor, introduction of two vector bases for constructing the reduced-order computational model, and simplification of the elastoacoustic model used for building the impedance of the liner. In this aeroacoustic problem, there are therefore many model uncertainties induced by modeling errors. It is known for a long time that model uncertainties cannot be taken into account by parametric probabilistic approaches. It is for this reason that the nonparametric probabilistic approach of model uncertainties was proposed in 2000 [49, 58, 59] and has given rise to numerous developments and validation works over the past 20 years. This approach has now become a standard method, which is, for example, implemented in commercial software such as MSC Nastran software.

Concepts, mathematical developments, and experimental validations can be found in [50, 60, 61] and also in [50, 56, 62, 63] for elastoacoustic problems, and for more recent advances, for instance, in [64, 65]. Because of model uncertainties induced by modeling errors, the boundary value problem that is constructed, from which the computational model is derived, does not correspond to the physical reality that is modeled. It is therefore necessary to generate a stochastic family of boundary value problems whose solution space is large enough to contain the targets such as experimental results. We recall that the family generated by a parametric approach does not allow the construction of such a family (see for example [50]). The so-called nonparametric probabilistic approach consists in generating this stochastic family by modeling operators of the boundary problems by random operators whose levels of statistical fluctuations, that is to say, the levels of uncertainty, are controlled by hyperparameters. The mathematical properties of the considered operators are preserved during the construction of the associated random operators. One thus generates a family of stochastic solutions, which obviously do not satisfy the initial boundary value problem by construction. If all the mathematical concepts and developments associated with the nonparametric probabilistic approach can be used for the aeroacoustic problem considered in this work, it is necessary to specify the algebraic properties of each random operator considered in order to use the appropriate subsets of random matrices and to build their probabilistic models. It should be noted that two types of nonparametric probabilistic approach can be used to model matrix-valued random impedances. The first allows causality to be taken into account explicitly, as proposed in [66] and in [50, 67, 68]. However, the implementation of this type of model is relatively complex and generates significant calculation costs for large dimensions (which is the case here). We have therefore preferred to use an approach a little less refined but simpler to implement and not generating significant numerical costs. These are the developments that are presented in this section.

\section{B. Construction of the probabilistic model of the reduced liner impedance matrix}

\section{Construction of the probabilistic model of random complex matrix $\left[Z^{\mathrm{NS}}(\omega)\right]$.}

For this construction, we use the polar decomposition (see Appendix) of deterministic complex matrix $\left[Z^{\text {NS }}(\omega)\right]$ defined by Eq (40] and then, we construct the complex random matrix $\left[\mathbf{Z}^{\mathrm{NS}}(\omega)\right]$. The positive frequency $\omega$ is assumed to be fixed.

(i) - Polar decomposition of complex matrix $\left[\mathbb{Z}^{\mathrm{NS}}(\omega)\right]$. It is assumed that the $\left(n_{\ell} \times n_{\ell}\right)$ complex matrix $\left[\mathbb{Z}^{\mathrm{NS}}(\omega)\right]$ is invertible. Therefore, matrix $\left[\mathbb{Z}^{\mathrm{NS}}(\omega)\right]$ can be written as

$$
\left[\mathbb{Z}^{\mathrm{NS}}(\omega)\right]=\left[U_{\mathbb{Z}}(\omega)\right]\left[T_{\mathbb{Z}}(\omega)\right],
$$

in which $\left[T_{\mathbb{Z}}(\omega)\right]$ is a positive-definite Hermitian $\left(n_{\ell} \times n_{\ell}\right)$ complex matrix and where $\left[U_{\mathbb{Z}}(\omega)\right]$ is a unitary $\left(n_{\ell} \times n_{\ell}\right)$ complex matrix such that $\left[U_{\mathbb{Z}}(\omega)\right]^{*}\left[U_{\mathbb{Z}}(\omega)\right]=\left[I_{n_{\ell}}\right]$.

(ii) - Probabilistic model of random complex matrix $\left[Z^{\mathrm{NS}}(\omega)\right]$. Let $\left[\mathbb{Z}^{\mathrm{NS}}(\omega)\right]$ be the invertible complex matrix for which its polar decomposition is defined by Eq. [41). The construction of the $\left(n_{\ell} \times n_{\ell}\right)$ complex random matrix $\left[\mathbf{Z}^{\mathrm{NS}}(\omega)\right]$, defined on a probability space $(\Theta, \mathcal{T}, \mathcal{P})$, associated with $\left[\mathbb{Z}^{\mathrm{NS}}(\omega)\right]$, is written as

$$
\left[\mathbf{Z}^{\mathrm{NS}}(\omega)\right]=\left[U_{\mathbb{Z}}(\omega)\right]\left[\mathbf{T}_{\mathbb{Z}}(\omega)\right]
$$


in which $\left[\mathbf{T}_{\mathbb{Z}}(\omega)\right]$ is a positive-definite Hermitian $\left(n_{\ell} \times n_{\ell}\right)$ complex random matrix, defined on $(\Theta, \mathcal{T}, \mathcal{P})$, such that,

$$
E\left\{\left\|\mathbf{T}_{\mathbb{Z}}(\omega)\right\|_{F}^{2}\right\}=\int_{\Theta}\left\|\mathbf{T}_{\mathbb{Z}}(\omega ; \theta)\right\|_{F}^{2} d \mathcal{P}(\theta)<+\infty,
$$

in which $E$ is the mathematical expectation. The probabilistic construction of random matrix $\left[\mathbf{T}_{\mathbb{Z}}(\omega)\right]$ is performed as follows. The Cholesky factorization of the positive-definite Hermitian matrix $\left[T_{\mathbb{Z}}(\omega)\right]$ is written as

$$
\left[T_{\mathbb{Z}}(\omega)\right]=\left[L_{T}^{\mathbb{Z}}(\omega)\right]^{*}\left[L_{T}^{\mathbb{Z}}(\omega)\right],
$$

in which $\left[L_{T}^{\mathbb{Z}}(\omega)\right]$ is an upper triangular $\left(n_{\ell} \times n_{\ell}\right)$ complex matrix with positive diagonal. The random matrix $\left[\mathbf{T}_{\mathbb{Z}}(\omega)\right]$ is then constructed as

$$
\left[\mathbf{T}_{\mathbb{Z}}(\omega)\right]=\left[L_{T}^{\mathbb{Z}}(\omega)\right]^{*}\left[\mathbf{G}_{\mathbb{Z}}^{\mathrm{NS}}\right]\left[L_{T}^{\mathbb{Z}}(\omega)\right]
$$

in which the random $\left(n_{\ell} \times n_{\ell}\right)$ real matrix $\left[\mathbf{G}_{\mathbb{Z}}^{\mathrm{NS}}\right]$ belongs to the set $\mathrm{SG}_{\mathcal{\varepsilon}}^{+}$of random matrices defined in Appendix. The probability distribution of random matrix $\left[\mathbf{G}_{\mathbb{Z}}^{\mathrm{NS}}\right]$ depends on a hyperparameter $\delta$, denoted by $\delta_{G, \mathbb{Z}}^{\mathrm{NS}}$, which allows the level of uncertainties to be controlled.

\section{Construction of the probabilistic model of positive-definite symmetric real random matrix $\left[Z_{I}^{\mathrm{S}}(\omega)\right]$.}

The positive-definite symmetric $\left(n_{\ell} \times n_{\ell}\right)$ real matrix $\left[\mathbb{Z}_{I}^{S}(\omega)\right]$, defined by Eq. [38), is modeled by a random matrix $\left[\mathbf{Z}_{I}^{\mathrm{S}}(\omega)\right]$ defined on a probability space $(\Theta, \mathcal{T}, \mathcal{P})$, which is assumed to be statistically independent of random matrix $\left[\mathbf{Z}^{\mathrm{NS}}(\omega)\right]$. The Cholesky factorization of $\left[\mathbb{Z}_{I}^{\mathrm{S}}(\omega)\right]$ is written as

$$
\left[\mathbb{Z}_{I}^{\mathrm{S}}(\omega)\right]=\left[L_{\mathbb{Z}}^{\mathrm{S}}(\omega)\right]^{T}\left[L_{\mathbb{Z}}^{\mathrm{S}}(\omega)\right]
$$

in which $\left[L_{\mathbb{Z}}^{\mathrm{S}}(\omega)\right]$ is an upper triangular $\left(n_{\ell} \times n_{\ell}\right)$ real matrix with positive diagonal. The random $\left(n_{\ell} \times n_{\ell}\right)$ complex matrix $\left[\mathbf{Z}_{I}^{\mathrm{S}}(\omega)\right]$ is then constructed as

$$
\left[\mathbf{Z}_{I}^{\mathrm{S}}(\omega)\right]=\left[L_{\mathbb{Z}}^{\mathrm{S}}(\omega)\right]^{T}\left[\mathbf{G}_{\mathbb{Z}}^{\mathrm{S}}\right]\left[L_{\mathbb{Z}}^{\mathrm{S}}(\omega)\right],
$$

in which $\left[\mathbf{G}_{\mathbb{Z}}^{\mathrm{S}}\right]$ is a random $\left(n_{\ell} \times n_{\ell}\right)$ real matrix that belongs to the ensemble $\mathrm{SG}_{\varepsilon}^{+}$defined in Appendix. The probability distribution of random matrix $\left[\mathbf{G}_{\mathbb{Z}}^{\mathrm{S}}\right]$ depends on a hyperparameter $\delta$, denoted by $\delta_{G, \mathbb{Z}}^{\mathrm{S}}$, which allows the level of uncertainties to be controlled.

\section{Probabilistic model of random matrix $[\boldsymbol{Z}(\omega)]$}

In the framework of the nonparametric approach of uncertainties, complex matrix [ $\mathbb{Z}(\omega)]$, defined by Eq. [33] and which is written as in Eq. [39), is then replaced by the random matrix,

$$
[\mathbf{Z}(\omega)]=\left[\mathbf{Z}^{\mathrm{NS}}(\omega)\right]+j\left[\mathbf{Z}_{I}^{\mathrm{S}}(\omega)\right],
$$

whose probability distribution depends on two hyperparameters $\delta_{G, \mathbb{Z}}^{\mathrm{NS}}$ and $\delta_{G, \mathbb{Z}}^{\mathrm{S}}$, which allow for controlling the level of uncertainties. In the application, we have chosen to define only one frequency-independent hyperparameter, $\delta_{Z}$, for controlling the level of uncertainties in the liner model, which is such that $\delta_{G, \mathbb{Z}}^{\mathrm{NS}}=\delta_{G, \mathbb{Z}}^{\mathrm{S}}=\delta_{Z}$.

\section{Construction of the probabilistic model of the reduced aeroacoustic matrix}

For $\omega$ fixed, the deterministic $\left(n_{\ell} \times n_{\ell}\right)$ complex matrix $[\mathbb{A}(\omega)]$, defined by Eq. [32], is modeled by a random matrix $[\mathbf{A}(\omega)]$, defined on probability space $(\Theta, \mathcal{T}, \mathcal{P})$, which is assumed to be statistical independent of random matrix $\mathbf{Z}(\omega)$.

(i) - Polar decomposition of complex matrix $[\mathbb{A}(\omega)]$. The polar decomposition (see Appendix) of the $\left(n_{\ell} \times n_{\ell}\right)$ complex matrix $[\mathbb{A}(\omega)]$ is written as

$$
[\mathbb{A}(\omega)]=\left[U_{\mathbb{A}}(\omega)\right]\left[T_{\mathbb{A}}(\omega)\right],
$$

in which $\left[U_{\mathbb{A}}(\omega)\right]$ is a unitary $\left(n_{\ell} \times n_{\ell}\right)$ complex matrix, such that $\left[U_{\mathbb{A}}(\omega)\right]^{*}\left[U_{\mathbb{A}}(\omega)\right]=\left[I_{n_{\ell}}\right]$, and where $\left[T_{\mathbb{A}}(\omega)\right]$ is a positive-definite Hermitian $\left(n_{\ell} \times n_{\ell}\right)$ complex matrix. 
(ii) - Probabilistic model of random complex matrix $[\boldsymbol{A}(\omega)]$. Matrix $[\mathbb{A}(\omega)]$ is modeled by the random matrix $[\mathbf{A}(\omega)]$ using the representation defined by Eq. [49], in which matrix $\left[T_{\mathbb{A}}(\omega)\right]$ is modeled by a random matrix $\left[\mathbf{T}_{\mathbb{A}}(\omega)\right]$ (as it has been performed in Section IV.B.1.(ii)). The Cholesky factorization of matrix $\left[T_{\mathbb{A}}(\omega)\right]$ is written as

$$
\left[T_{\mathbb{A}}(\omega)\right]=\left[L_{T}^{\mathbb{A}}(\omega)\right]^{*}\left[L_{T}^{\mathbb{A}}(\omega)\right],
$$

in which $\left[L_{T}^{\mathbb{A}}(\omega)\right]$ is an upper triangular $\left(n_{\ell} \times n_{\ell}\right)$ complex matrix with positive diagonal. The random complex matrix $\left[\mathbf{T}_{\mathbb{A}}(\omega)\right]$, is then constructed as

$$
\left[\mathbf{T}_{\mathbb{A}}(\omega)\right]=\left[L_{T}^{\mathbb{A}}(\omega)\right]^{*}\left[\mathbf{G}_{\mathbb{A}}\right]\left[L_{T}^{\mathbb{A}}(\omega)\right],
$$

in which the random $\left(n_{\ell} \times n_{\ell}\right)$ real matrix $\left[\mathbf{G}_{\mathbb{A}}\right]$ belongs to ensemble $\mathrm{SG}_{\varepsilon}^{+}$defined in Appendix. The probability distribution of $\left[\mathbf{G}_{\mathbb{A}}\right]$ depends on a hyperparameter $\delta$ that is denoted by $\delta_{A}$ that allows the level of uncertainties to be controlled. Consequently, random matrix $[\mathbf{A}(\omega)]$ is written as

$$
[\mathbf{A}(\omega)]=\left[U_{\mathbb{A}}(\omega)\right]\left[\mathbf{T}_{\mathbb{A}}(\omega)\right],
$$

for which the hyperparameter is $\delta_{A}$ that is independent of $\omega$.

\section{Parametric probabilistic modeling of the fan acoustic excitation}

There are two possible approaches for constructing the parametric probabilistic model of the fan excitation. The first one consists in directly considering the modal components $\left\{c_{\alpha}^{+}(\omega), \alpha=1, \ldots, N_{d}\right\}$ of the duct modes, while the second one consists in describing the probabilistic model for the modal intensity $\left\{I_{\alpha}^{+}(\omega), \alpha=1, \ldots, N_{d}\right\}$ and using the expression of $c_{\alpha}^{+}(\omega)$ as a function of $I_{\alpha}^{+}(\omega)$. In the present paper, we only present the latter. In addition, the reader will find the relation between $c_{\alpha}^{+}(\omega)$ and $I_{\alpha}^{+}(\omega)$ in Appendix B of the Dangla thesis [69] (this relation is not given because the required explanations are long enough and relatively fastidious).

Probabilistic model of $I_{\alpha}^{+}(\omega)$. For civil aircraft applications, a deterministic mean model, $\underline{I}_{\alpha}^{+}(\omega)$, of the modal intensity is often used by the manufacturers and this mean model has to be accounted for in the construction of the probabilistic model of $I_{\alpha}^{+}(\omega)$. Consequently, $\underline{I}_{\alpha}^{+}(\omega)$ is chosen as the statistical mean value of the random variable $I_{\alpha}^{+}(\omega)$. For the construction of the probability distribution of the modal intensity, the available information are the following: $I_{\alpha}^{+}(\omega)$ is a positive-valued random variable, its mean value is $\underline{I}_{\alpha}^{+}(\omega)$, and the support of its probability distribution is the given positive interval $\mathcal{J}_{\alpha}(\omega)=\left[\underline{I}_{\alpha}^{+}(\omega)\left(1-\sigma_{\alpha}(\omega)\right), \underline{I}_{\alpha}^{+}(\omega)\left(1+\sigma_{\alpha}(\omega)\right)\right] \subset \mathbb{R}^{+}$depending on $\alpha$ and on a positive hyperparameter $0<\sigma_{\alpha}(\omega)<1$. It should be noted that no information is assumed to be available concerning the statistical dependence of the random variables $I_{1}^{+}(\omega), \ldots, I_{N_{d}}^{+}(\omega)$. Using the MaxEnt principle of Information Theory, it is concluded that the random variables $I_{1}^{+}(\omega), \ldots, I_{N_{d}}^{+}(\omega)$ are statistically independent and the probability distribution of each $I_{\alpha}^{+}(\omega)$ is uniform with support $\mathcal{J}_{\alpha}(\omega)$ and with mean value $\underline{I}_{\alpha}^{+}(\omega)$. Therefore, the random variable $I_{\alpha}^{+}(\omega)$ can be written as

$$
I_{\alpha}^{+}(\omega)=\underline{I}_{\alpha}^{+}(\omega)\left(1+\sigma_{\alpha}(\omega) U_{\alpha}(\omega)\right) \quad, \quad 0<\sigma_{\alpha}(\omega)<1,
$$

in which $U_{\alpha}(\omega)$ is a centered uniform random variable on the interval $[-1,1]$.

\section{E. Construction of the SROM by using the nonparametric probabilistic approach of uncertainties}

As previously explained, the nonparametric probabilistic approach of both the parametric uncertainties and the model uncertainties induced by modeling errors in the computational model of the acoustic radiation of an inlet consists in modeling matrix $[\mathbb{Z}(\omega)]$ defined by Eq. $[33$ by the random complex matrix $[\mathbf{Z}(\omega)]$ defined by Eq. (48) and $[\mathbb{A}(\omega)]$ defined by Eq. 32 by the random complex matrix $[\mathbf{A}(\omega)]$ defined by Eq. 52 . The introduction of uncertainties on the fan acoustic excitation consists in modeling $\mathbf{c}^{+}(\omega)=\left(c_{1}^{+}(\omega), \ldots, c_{N_{d}}^{+}(\omega)\right)$ by the random complex vector $\mathbf{C}^{+}(\omega)=\left(C_{1}^{+}(\omega), \ldots, C_{N_{d}}^{+}(\omega)\right)$ that is expressed as a function of $\mathbf{I}^{+}(\omega)=\left(I_{1}^{+}(\omega), \ldots, I_{N_{d}}^{+}(\omega)\right)$ (see Section IV.D. From Eqs. (37) and 31), it can be deduced that the random vector $\mathbf{Q o I}(\omega)$ depending on $\omega$ is such that

$$
\operatorname{QoI}(\omega)=\left[\mathbb{F}_{\mathrm{qoI}}(\omega)\right] \mathbf{C}^{+}(\omega)-\left[\mathbb{A}_{\mathrm{qoI}}(\omega)\right] \mathbf{Q}(\omega),
$$

in which the random vector $\mathbf{Q}(\omega)$ is such that

$$
([\mathbf{A}(\omega)]+[\mathbf{Z}(\omega)]) \mathbf{Q}(\omega)=[\mathbb{B}(\omega)] \mathbf{C}^{+}(\omega)
$$




\section{F. Solving the stochastic matrix equation and constructing the confidence regions}

The frequency band of analysis $\left[\omega_{\min }, \omega_{\max }\right]$ is sampled in $n_{\omega}$ points $\left\{\omega_{1}, \ldots \omega_{n_{\omega}}\right\}$. For each $\omega=\omega_{j}$, the Monte Carlo method is used for solving the SROM. It consists in computing a set of $v_{s}$ independent realizations, with $v_{s}$ sufficiently large, of the random quantities of interest by using Eqs. (54) and (55). From this set of independent realizations, estimates of the statistics for the quantities of interest are computed, such as probability density functions, moments (means, standard deviations), confidence intervals. The convergence of the estimates are analyzed with respect to $v_{s}$.

\section{Appendix}

Polar decomposition of a complex matrix

An invertible $\left(n_{\ell} \times n_{\ell}\right)$ complex matrix $[\mathbb{Q}(\omega)]$ can be written (polar decomposition) as

$$
[\mathbb{Q}(\omega)]=\left[U_{\mathbb{Q}}(\omega)\right]\left[T_{\mathbb{Q}}(\omega)\right],
$$

in which $\left[U_{\mathbb{Q}}(\omega)\right]$ is a unitary $\left(n_{\ell} \times n_{\ell}\right)$ complex matrix such that $\left[U_{\mathbb{Q}}(\omega)\right]^{*}\left[U_{\mathbb{Q}}(\omega)\right]=\left[I_{n_{\ell}}\right]$ (with $\left[I_{n_{\ell}}\right]$ the $\left(n_{\ell} \times n_{\ell}\right)$ identity matrix) and where the matrix $\left[T_{\mathbb{Q}}(\omega)\right]$ belongs to the set $\mathbb{M}_{n_{\ell}}^{+}(\mathbb{C})$ of all the positive-definite Hermitian $\left(n_{\ell} \times n_{\ell}\right)$ complex matrices.

The construction of the polar decomposition defined by Eq. [56) can be done as follows. Let $\left[H_{\mathbb{Q}}(\omega)\right]$ be the matrix defined by $\left[H_{\mathbb{Q}}(\omega)\right]=[\mathbb{Q}(\omega)]^{*}[\mathbb{Q}(\omega)]$. Since $[\mathbb{Q}(\omega)]$ is assumed to be invertible, then the matrix $\left[H_{\mathbb{Q}}(\omega)\right]$ belongs to $\mathbb{M}_{n_{\ell}}^{+}(\mathbb{C})$. The spectral decomposition of $\left[H_{\mathbb{Q}}(\omega)\right]$ is $\left[H_{\mathbb{Q}}(\omega)\right]=\left[\Phi_{\mathbb{Q}}(\omega)\right]\left[S_{\mathbb{Q}}(\omega)\right]\left[\Phi_{\mathbb{Q}}(\omega)\right]^{*}$, in which $\left[S_{\mathbb{Q}}(\omega)\right]$ is the diagonal $\left(n_{\ell} \times n_{\ell}\right)$ real matrix of the positive eigenvalues of $[H(\omega)]$ and where $\left[\Phi_{\mathbb{Q}}(\omega)\right]$ is the $\left(n_{\ell} \times n_{\ell}\right)$ complex matrix of the eigenvectors such that $\left[\Phi_{\mathbb{Q}}(\omega)\right]^{*}[\Phi(\omega)]=\left[\Phi_{\mathbb{Q}}(\omega)\right]\left[\Phi_{\mathbb{Q}}(\omega)\right]^{*}=\left[I_{n_{\ell}}\right]$. Note that diagonal matrix $\left[S_{\mathbb{Q}}(\omega)\right]$ can also be viewed as the diagonal matrix of the singular values of complex matrix $[\mathbb{Q}(\omega)]$. The matrix $\left[T_{\mathbb{Q}}(\omega)\right]$ in Eq. (56) is calculated by $\left[T_{\mathbb{Q}}(\omega)\right]=\left[\Phi_{\mathbb{Q}}(\omega)\right]\left[S_{\mathbb{Q}}(\omega)\right]^{1 / 2}\left[\Phi_{\mathbb{Q}}(\omega)\right]^{*}$. It can be seen that $\left[T_{\mathbb{Q}}(\omega)\right]$ is in $\mathbb{M}_{n_{\ell}}^{+}(\mathbb{C})$. Finally, the matrix $\left[U_{\mathbb{Q}}(\omega)\right]$ in in Eq. ${ }^{56}$ is calculated by $\left[U_{\mathbb{Q}}(\omega)\right]=[\mathbb{Q}(\omega)]\left[T_{\mathbb{Q}}(\omega)\right]^{-1}$. It can be verified that $\left[U_{\mathbb{Q}}(\omega)\right]^{*}\left[U_{\mathbb{Q}}(\omega)\right]=\left[I_{n_{\ell}}\right]$.

\section{Definition and construction of the set $\mathrm{SG}_{\varepsilon}^{+}$of random matrices [50]}

All the matrices are independent of the frequency parameter $\omega$. Let $\varepsilon>0$ be a fixed positive small parameter $(\varepsilon \ll 1)$. A random $\left(n_{\ell} \times n_{\ell}\right)$ real matrix $[\mathbf{G}]$, defined on a probability space $(\Theta, \mathcal{T}, \mathcal{P})$, belongs to ensemble $\mathrm{SG}_{\varepsilon}^{+}$, if it is a random matrix with values in $\mathbb{M}_{n_{\ell}}^{+}(\mathbb{R})$, which is written as

$$
[\mathbf{G}]=\frac{1}{1+\varepsilon}\left\{\left[\mathbf{G}_{0}\right]+\varepsilon\left[I_{n_{\ell}}\right]\right\},
$$

in which $\left[\mathbf{G}_{0}\right]$ is a random $\left(n_{\ell} \times n_{\ell}\right)$ real matrix with values in $\mathbb{M}_{n_{\ell}}^{+}(\mathbb{R})$, defined on $(\Theta, \mathcal{T}, \mathcal{P})$, which belongs to the set $\mathrm{SG}_{0}^{+}$of random matrices defined in [50]. Random matrix $\left[\mathbf{G}_{0}\right]$ has been constructed using the Maximum Entropy principle under the constraints defined by the following available information,

$$
E\left\{\left[\mathbf{G}_{0}\right]\right\}=\left[I_{n_{\ell}}\right] \quad, \quad E\left\{\log \left(\operatorname{det}\left[\mathbf{G}_{0}\right]\right)\right\}=v_{G_{0}} .
$$

in which $v_{G_{0}}$ is any constant such that $\left|v_{G_{0}}\right|<+\infty$. The obtained probability distribution of $\left[\mathbf{G}_{0}\right]$ is not Gaussian, depends on constant $v_{G_{0}}$, and is reparameterized using the dispersion parameter $\delta$, defined by,

$$
\delta=\left\{\frac{E\left\{\left\|\left[\mathbf{G}_{0}\right]-E\left\{\left[\mathbf{G}_{0}\right]\right\}\right\|_{F}^{2}\right\}}{\left\|E\left\{\left[\mathbf{G}_{0}\right]\right\}\right\|_{F}^{2}}\right\}^{1 / 2}=\left\{\frac{1}{n_{\ell}} E\left\{\left\|\left[\mathbf{G}_{0}\right]-\left[I_{n_{\ell}}\right]\right\|_{F}^{2}\right\}^{1 / 2} .\right.
$$

Consequently, the probability distribution of the non-Gaussian random matrix $\left[\mathbf{G}_{0}\right]$ depends only on one hyperparameter that is $\delta$. This hyperparameter allows for controlling the level of statistical fluctuations, that is to say, for controlling the level of uncertainties. Using $E\left\{\left[\mathbf{G}_{0}\right]\right\}=\left[I_{n_{\ell}}\right]$, Eq. [57) yields $E\{[\mathbf{G}]\}=\left[I_{n_{\ell}}\right]$. For $\theta$ fixed in $\Theta$, the realization $\left[\mathbf{G}_{0}(\theta)\right]$ is computed by using the following representation of random matrix $\left[\mathbf{G}_{0}\right]$,

$$
\left[\mathbf{G}_{0}\right]=[\mathbf{L}]^{T}[\mathbf{L}],
$$

in which $[\mathbf{L}]$ is an upper triangular random matrix with values in $\mathbb{M}_{n_{\ell}}(\mathbb{R})$ such that, 
1) the random variables $\left\{[\mathbf{L}]_{j j^{\prime}}, j \leq j^{\prime}\right\}$ are mutually independent.

2) for $j<j^{\prime}$, we have $[\mathbf{L}]_{j j^{\prime}}=\sigma \mathcal{G}_{j j^{\prime}}$ in which $\sigma=\delta\left(n_{\ell}+1\right)^{-1 / 2}$ and where $\mathcal{G}_{j j^{\prime}}$ is a real-valued Gaussian random variable with zero mean and with a variance that is equal to 1 .

3) for $j=j^{\prime}$, we have $[\mathbf{L}]_{j j}=\sigma \sqrt{2 \mathcal{V}_{j}}$ where $\mathcal{V}_{j}$ is a positive-valued Gamma random variable whose probability density function with respect to $d v$ is written as

$$
p_{\mathcal{V}_{j}}(v)=\mathbb{1}_{\mathbb{R}^{+}}(v) \frac{1}{\Gamma\left(\frac{n_{\ell}+1}{2(\delta)^{2}}+\frac{1-j}{2}\right)} v^{\frac{n_{\ell}+1}{2(\delta)^{2}}-\frac{1+j}{2}} e^{-v} .
$$

The random variable $\mathcal{V}_{j}$ can be rewritten as the nonlinear transformation $h\left(\mathcal{G}_{j j} ; n_{\ell} ; \delta\right)$ of a real-valued Gaussian random variable $\mathcal{G}_{j j}$ with zero mean and with a variance that is equal to 1 .

4) The Gaussian random variables $\left\{\mathcal{G}_{j j^{\prime}}, 1 \leq j \leq j^{\prime} \leq n_{\ell}\right\}$ are statistically independent.

Remarks.

1) The algebraic representation defined by Eq. 60 shows that although the entries $\left\{[\mathbf{L}]_{j j^{\prime}}, j \leq j^{\prime}\right\}$ of $[\mathbf{L}]$ are mutually independent, the entries $\left\{\left[\mathbf{G}_{0}\right]_{j j^{\prime}}, j \leq j^{\prime}\right\}$ of $\left[\mathbf{G}_{0}\right]$ are mutually dependent.

2) The diagonal entries $[\mathbf{L}]_{j j}, j=1, \ldots, n_{\ell}$ of random matrix $[\mathbf{L}]$ depend on $j$.

\section{References}

[1] Lighthill, M. J., "On sound generated aerodynamically. I. General theory,” Proceedings of the Royal Society of London A: Mathematical, Physical and Engineering Sciences, Vol. 211, The Royal Society, 1952, pp. 564-587.

[2] Lighthill, M. J., Waves in fluids, Cambridge university press, 2001.

[3] Hirschberg, A., and Rienstra, S. W., "An introduction to aeroacoustics," Eindhoven university of technology, 2004.

[4] Landau, L. D., and Lifshitz, E., Fluid Mechanics: Vol 6, Elsevier, 2013.

[5] Mohring, W., "A well posed acoustic analogy based on a moving acoustic medium," arXiv preprint arXiv:1009.3766, 2010.

[6] Bruneau, M., and Scelo, T., Fundamentals of Acoustics, ISTE, Wiley, 2010. URL https://books.google.fr/books?id= 8W4TtWhP_nEC

[7] Pierce, A. D., and Beyer, R. T., "Acoustics: An Introduction to Its Physical Principles and Applications. 1989 Edition,” , 1990.

[8] Jones, M., Watson, W., and Parrott, T., "Benchmark data for evaluation of aeroacoustic propagation codes with grazing flow," 11th AIAA/CEAS Aeroacoustics Conference, 2005, p. 2853.

[9] Tam, C. K., “Computational aeroacoustics-Issues and methods,” AIAA journal, Vol. 33, No. 10, 1995 , pp. $1788-1796$. doi:https://doi.org/10.2514/3.12728.

[10] Tam, C. K., and Dong, Z., "Radiation and outflow boundary conditions for direct computation of acoustic and flow disturbances in a nonuniform mean flow," Journal of computational acoustics, Vol. 4, No. 02, 1996, pp. 175-201. doi: https://doi.org/10.1142/S0218396X96000040.

[11] Tam, C. K., "Computational aeroacoustics: an overview of computational challenges and applications," International Journal of Computational Fluid Dynamics, Vol. 18, No. 6, 2004, pp. 547-567. doi:https://doi.org/10.1080/10618560410001673551.

[12] Tam, C. K., "Advances in numerical boundary conditions for computational aeroacoustics," Journal of Computational Acoustics, Vol. 6, No. 04, 1998, pp. 377-402. doi:https://doi.org/10.1142/S0218396X98000259.

[13] Zhang, Y., Chen, H., Wang, K., and Wang, M., "Aeroacoustic prediction of a multi-element airfoil using wall-modeled large-eddy simulation," AIAA Journal, 2017, pp. 4219-4233. doi:https://doi.org/10.2514/1.J055853.

[14] Freund, J. B., "Proposed inflow/outflow boundary condition for direct computation of aerodynamic sound," AIAA journal, Vol. 35, No. 4, 1997, pp. 740-742. doi:https://doi.org/10.2514/2.167.

[15] Fahy, F. J., and Gardonio, P., Sound and structural vibration: radiation, transmission and response, Academic press, 2007.

[16] Hirsch, C., Numerical Computation of Internal and External Flows, Fundamentals of Numerical Discretization, Numerical Computation of Internal and External Flows, Wiley, 1991. URL https://books.google.fr/books?id=pswYAQAAIAA] 
[17] Wagner, C., Hüttl, T., and Sagaut, P., Large-eddy simulation for acoustics, Vol. 20, Cambridge University Press, 2007.

[18] Manoha, E., Troff, B., and Sagaut, P., “Trailing-edge noise prediction using large-eddy simulation and acoustic analogy," AIAA journal, Vol. 38, No. 4, 2000, pp. 575-583. doi:https://doi.org/10.2514/2.1015.

[19] Bailly, C., and Juvé, D., "Aeroacoustic simulations and stochastic approach using linearized Euler's equations," The Journal of the Acoustical Society of America, Vol. 105, No. 2, 1999, pp. 1066-1066. doi:https://doi.org/10.1121/1.425067.

[20] Bailly, C., and Juve, D., "Numerical solution of acoustic propagation problems using linearized Euler equations," AIAA journal, Vol. 38, No. 1, 2000, pp. 22-29. doi:https://doi.org/10.2514/2.949.

[21] Astley, R., Sugimoto, R., and Mustafi, P., "Computational aero-acoustics for fan duct propagation and radiation. Current status and application to turbofan liner optimisation," Journal of Sound and Vibration, Vol. 330, No. 16, 2011, pp. 3832-3845. doi:https://doi.org/10.1016/j.jsv.2011.03.022.

[22] Lidoine, S., "Approches théoriques du problème du rayonnement acoustique par une entrée d'air de turboréacteur: Comparaisons entre différentes méthodes analytiques et numériques," Ph.D. thesis, Ecully, Ecole centrale de Lyon, 2002.

[23] Guess, A., "Calculation of perforated plate liner parameters from specified acoustic resistance and reactance," Journal of Sound and Vibration, Vol. 40, No. 1, 1975, pp. 119-137. doi:https://doi.org/10.1016/S0022-460X(75)80234-3.

[24] Parrott, T. L., and Jones, M. G., "Parallel-element liner impedances for improved absorption of broadband sound in ducts," Noise Control Engineering Journal, Vol. 43, No. 6, 1995, pp. 183-195. doi:https://doi.org/10.3397/1.2828379.

[25] Jones, M., Tracy, M., Watson, W., and Parrott, T., "Effects of liner geometry on acoustic impedance," 8th AIAA/CEAS Aeroacoustics Conference \& Exhibit, 2002, p. 2446.

[26] Jones, M., Parrott, T., and Watson, W., "Uncertainty and sensitivity analyses of a two-parameter impedance prediction model," 14th AIAA/CEAS Aeroacoustics Conference (29th AIAA Aeroacoustics Conference), 2008, p. 2928.

[27] Brown, M., Jones, M., and Watson, W., "Uncertainty analysis of the grazing flow impedance tube," 18th AIAA/CEAS Aeroacoustics Conference (33rd AIAA Aeroacoustics Conference), 2012, p. 2296.

[28] Primus, J., Piot, E., and Simon, F., "An adjoint-based method for liner impedance eduction: Validation and numerical investigation," Journal of Sound and Vibration, Vol. 332, No. 1, 2013, pp. 58-75. doi:https://doi.org/10.1016/j.jsv.2012.07.051.

[29] Tam, C. K., Pastouchenko, N., Jones, M. G., and Watson, W. R., "Experimental validation of numerical simulation for an acoustic liner in grazing flow," 19th AIAA/CEAS Aeroacoustics Conference, 2013, p. 222.

[30] Zhao, D., Ang, L., and Ji, C., "Numerical and experimental investigation of the acoustic damping effect of single-layer perforated liners with joint bias-grazing flow," Journal of Sound and Vibration, Vol. 342, 2015, pp. 152-167. doi:https: //doi.org/10.1016/j.jsv.2014.12.035.

[31] Zhao, D., Ji, C., Li, J., and Ang, L., "Experimental comparison of noise dissipation effects of single-and double-layer acoustic liners," Applied Acoustics, Vol. 141, 2018, pp. 281-292. doi:https://doi.org/10.1016/j.apacoust.2018.07.024.

[32] Zhao, D., Sun, Y., Ni, S., Ji, C., and Sun, D., "Experimental and theoretical studies of aeroacoustics damping performance of a biasflow perforated orifice," Applied Acoustics, Vol. 145, 2019, pp. 328-338. doi:https://doi.org/10.1016/j.apacoust.2018.10.025.

[33] W. Tam, C. K., and Kurbatskii, K. A., "Microfluid dynamics and acoustics of resonant liners," AIAA journal, Vol. 38, No. 8, 2000, pp. 1331-1339. doi:https://doi.org/10.2514/2.1132.

[34] Roche, J.-M., Leylekian, L., Delattre, G., and Vuillot, F., "Aircraft fan noise absorption: DNS of the acoustic dissipation of resonant liners," 15th AIAA/CEAS Aeroacoustics Conference (30th AIAA Aeroacoustics Conference), 2009, p. 3146.

[35] Mendez, S., and Eldredge, J. D., "Acoustic modeling of perforated plates with bias flow for large-eddy simulations," Journal of Computational Physics, Vol. 228, No. 13, 2009, pp. 4757-4772. doi:https://doi.org/10.1016/j.jcp.2009.03.026.

[36] Marx, D., and Aurégan, Y., "Effect of turbulent eddy viscosity on the unstable surface mode above an acoustic liner," Journal of Sound and Vibration, Vol. 332, No. 15, 2013, pp. 3803-3820. doi:https://doi.org/10.1016/j.jsv.2013.02.005.

[37] Zhang, Q., and Bodony, D. J., "Numerical investigation of a honeycomb liner grazed by laminar and turbulent boundary layers," Journal of Fluid Mechanics, Vol. 792, 2016, pp. 936-980. doi:https://doi.org/10.1017/jfm.2016.79. 
[38] Duchaine, F., "Sensitivity Analysis of Heat Transfer in a Honeycomb Acoustic Liner to Inlet Conditions With Large Eddy Simulation," ASME Turbo Expo 2017: Turbomachinery Technical Conference and Exposition, American Society of Mechanical Engineers, 2017, pp. V05BT22A002-V05BT22A002.

[39] Ramdani, S., Yamasaki, N., Inokuchi, Y., and Ishii, T., "Large Eddy Simulation of Conventional and Bias Flow Acoustic Liners," ASME Turbo Expo 2017: Turbomachinery Technical Conference and Exposition, American Society of Mechanical Engineers, 2017, pp. V02CT43A007-V02CT43A007.

[40] Eldredge, J., Bodony, D., and Shoeybi, M., "Numerical investigation of the acoustic behavior of a multi-perforated liner," 13th AIAA/CEAS Aeroacoustics Conference (28th AIAA Aeroacoustics Conference), 2007, p. 3683.

[41] Pascal, L., Piot, E., and Casalis, G., "A new implementation of the extended Helmholtz resonator acoustic liner impedance model in time domain CAA," Journal of Computational Acoustics, Vol. 24, No. 01, 2016, p. 1550015. doi:https://doi.org/10. 1142/S0218396X15500150.

[42] Zhang, Q., and Bodony, D. J., "Numerical simulation of two-dimensional acoustic liners with high-speed grazing flow," AIAA journal, Vol. 49, No. 2, 2011, pp. 365-382. doi:https://doi.org/10.2514/1.J050597.

[43] Robinson, J., and Watson, W., "Performance of a checkerboard liner with uncertain impedances," 11th AIAA/CEAS Aeroacoustics Conference, 2005, p. 2850.

[44] Lafronza, L., McAlpine, A., Keane, A., and Astley, J., "Response surface method optimization of uniform and axially segmented duct acoustics liners," Journal of Aircraft, Vol. 43, No. 4, 2006, pp. 1089-1102. doi:https://doi.org/10.2514/1.17727.

[45] Di Francescantonio, P., Casalino, D., and De Mercato, L., "Aeroacoustic design of aero-engine intake liners," 11th AIAA/CEAS Aeroacoustics Conference, 2005, p. 2942.

[46] Van Den Nieuwenhof, B., Detandt, Y., Lielens, G., Rosseel, E., Soize, C., Dangla, V., Kassem, M., and Mosson, A., "Optimal Design of the Acoustic Treatments Damping the Noise Radiated by a Turbo-Fan Engine," 23rd AIAA/CEAS Aeroacoustics Conference, 2017, p. 4035.

[47] Myers, M., "On the acoustic boundary condition in the presence of flow," Journal of Sound and Vibration, Vol. 71, No. 3, 1980, pp. 429-434. doi:https://doi.org/10.1016/0022-460X(80)90424-1.

[48] Brambley, E. J., "Well-posed boundary condition for acoustic liners in straight ducts with flow," AIAA journal, Vol. 49, No. 6, 2011, pp. 1272-1282. doi:https://doi.org/10.2514/1.J050723.

[49] Soize, C., "A nonparametric model of random uncertainties for reduced matrix models in structural dynamics," Probabilistic engineering mechanics, Vol. 15, No. 3, 2000, pp. 277-294. doi:https://doi.org/10.1016/S0266-8920(99)00028-4.

[50] Soize, C., Uncertainty Quantification. An Accelerated Course with Advanced Applications in Computational Engineering, Vol. Interdisciplinary Applied Mathematics, Springer, 2017.

[51] Ghanem, R., Higdon, D., and Owhadi, H., Handbook of Uncertainty Quantification, Vol. 1 to 3, Springer, Cham, Switzerland, 2017. doi:https://doi.org/10.1007/978-3-319-12385-1.

[52] Free Field Technologies, “Actran 12 User’s Guide,”, 2011.

[53] Van Antwerpen, B., Detandt, Y., Copiello, D., Rosseel, E., and Gaudry, E., "Performance improvements and new solution strategies of Actran/TM for nacelle simulations," 20th AIAA/CEAS Aeroacoustics Conference, 2014, p. 2315.

[54] van Den Nieuwenhof, B., Detandt, Y., Lielens, G., Rosseel, E., Soize, C., Dangla, V., Kassem, M., and Mosson, A., "Optimal Design of the Acoustic Treatments Damping the Noise Radiated by a Turbo-Fan Engine," 23rd AIAA/CEAS Aeroacoustics Conference, 2017, p. 4035.

[55] Rienstra, S. W., "Fundamentals of duct acoustics," Von Karman Institute Lecture Notes, 2015.

[56] Ohayon, R., and Soize, C., Advanced computational vibroacoustics: reduced-order models and uncertainty quantification, Cambridge University Press, 2014.

[57] Rubinstein, R. Y., and Kroese, D. P., Simulation and the Monte Carlo method, Vol. 10, John Wiley \& Sons, 2016.

[58] Soize, C., "Random matrix theory and non-parametric model of random uncertainties in vibration analysis," Journal of sound and vibration, Vol. 263, No. 4, 2003, pp. 893-916. doi:https://doi.org/10.1016/S0022-460X(02)01170-7. 
[59] Soize, C., "Random matrix theory for modeling uncertainties in computational mechanics," Computer methods in applied mechanics and engineering, Vol. 194, No. 12-16, 2005, pp. 1333-1366. doi:https://doi.org/10.1016/j.cma.2004.06.038.

[60] Soize, C., "A comprehensive overview of a non-parametric probabilistic approach of model uncertainties for predictive models in structural dynamics," Journal of sound and vibration, Vol. 288, No. 3, 2005, pp. 623-652. doi:https://doi.org/10.1016/j.jsv. 2005.07.009.

[61] Soize, C., "Random matrix models and nonparametric method for uncertainty quantification," Handbook of Uncertainty Quantification, Vol. 1, edited by R. Ghanem, D. Higdon, and H. Owhadi, Springer, Cham, Switzerland, 2017, pp. 219-287. doi:https://doi.org/10.1007/978-3-319-11259-6_5-1.

[62] Soize, C., "Random Matrices in Structural Acoustics," New Directions in Linear Acoustics and Vibration: Quantum Chaos, Random Matrix Theory and Complexity, 2010, p. 206.

[63] Ohayon, R., and Soize, C., "Computational vibroacoustics in low-and medium-frequency bands: damping, ROM, and UQ modeling,” Applied Sciences, Vol. 7, No. 6, 2017, p. 586. doi:https://doi.org/10.3390/app7060586.

[64] Arnst, M., and Soize, C., "Identification and sampling of Bayesian posteriors of high-dimensional symmetric positive-definite matrices for data-driven updating of computational models," Computer Methods in Applied Mechanics and Engineering, Vol. 352, 2019, pp. 300-323. doi:https://doi.org/10.1016/j.cma.2019.04.025.

[65] Akkaoui, Q., Capiez-Lernout, E., Soize, C., and Ohayon, R., "Uncertainty quantification for dynamics of geometrically nonlinear structures coupled with internal acoustic fluids in presence of sloshing and capillarity," Journal of Fluids and Structures, Vol. 94, 2020, p. 102966. doi:https://doi.org/10.1016/j.jfluidstructs.2020.102966.

[66] Cottereau, R., Clouteau, D., and Soize, C., "Construction of a probabilistic model for impedance matrices," Computer Methods in Applied Mechanics and Engineering, Vol. 196, No. 17-20, 2007, pp. 2252-2268. doi:https://doi.org/10.1016/j.cma.2006.12.001.

[67] Soize, C., and Poloskov, I. E., "Time-domain formulation in computational dynamics for linear viscoelastic media with model uncertainties and stochastic excitation," Computers \& Mathematics with Applications, Vol. 64, No. 11, 2012 , pp. 3594-3612. doi:https://doi.org/10.1016/j.camwa.2012.09.010.

[68] Capillon, R., Desceliers, C., and Soize, C., "Uncertainty quantification in computational linear structural dynamics for viscoelastic composite structures," Computer Methods in Applied Mechanics and Engineering, Vol. 305, 2016 , pp. 154-172. doi:https://doi.org/10.1016/j.cma.2016.03.012.

[69] Dangla, V., "Robust design of acoustic treatment for nacelle noise reduction using computational aeroacoustics and uncertainty quantification,” Ph.D. thesis, Université Gustave Eiffel, 2020. URL https://tel . archives-ouvertes . fr/tel-03115688/ document 